\title{
HAT-P-58b-HAT-P-64b: Seven Planets Transiting Bright Stars*
}

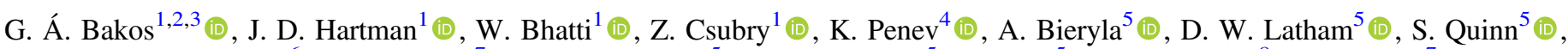
L. A. Buchhave ${ }^{6}$ (1) , G. Kovács ${ }^{7}$, Guillermo Torres ${ }^{5}$ (1) , R. W. Noyes ${ }^{5}$, E. Falco ${ }^{5}$ (1) Bence Béky ${ }^{8}$, T. Szklenár ${ }^{7}$ (1),

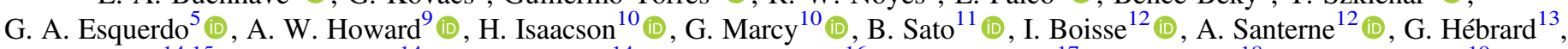

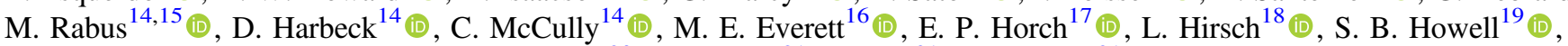

C. X. Huang ${ }^{20}$ (1) J. Lázár ${ }^{21}$, I. Papp ${ }^{21}$, and P. Sári ${ }^{21}$

${ }^{1}$ Department of Astrophysical Sciences, Princeton University, NJ 08544, USA; gbakos@astro.princeton.edu

${ }^{2}$ MTA Distinguished Guest Fellow, Konkoly Observatory, Research Centre for Astronomy and Earth Sciences, H-1121 Budapest, Konkoly Thege Miklósút 15-17, Hungary

${ }^{3}$ Institute for Advanced Study, 1 Einstein Drive, Princeton, NJ 08540, USA

${ }^{4}$ Department of Physics, University of Texas at Dallas, Richardson, TX 75080, USA

${ }^{5}$ Center for Astrophysics|Harvard \& Smithsonian, 60 Garden Street, Cambridge, MA 02138, USA

${ }^{6}$ DTU Space, National Space Institute, Technical University of Denmark, Elektrovej 328, DK-2800 Kgs. Lyngby, Denmark

${ }^{7}$ Konkoly Observatory, Research Centre for Astronomy and Earth Sciences, H-1121 Budapest, Konkoly Thege Miklósút 15-17, Hungary

${ }^{8}$ Google, Googleplex, 1600 Amphitheatre Parkway, Mountain View, CA 94043, USA

${ }^{9}$ Department of Astronomy, California Institute of Technology, Pasadena, CA, USA

${ }^{10}$ Department of Astronomy, University of California, Berkeley, CA, USA

${ }^{11}$ Department of Earth and Planetary Sciences, Tokyo Institute of Technology, 2-12-1 Ookayama, Meguro-ku, Tokyo 152-8551, Japan

${ }^{12}$ Aix Marseille Université, CNRS, LAM (Laboratoire d'Astrophysique de Marseille) UMR 7326, F-13388, Marseille, France

${ }^{13}$ Institut d'Astrophysique de Paris, UMR7095 CNRS, Université Pierre \& Marie Curie, 98bis boulevard Arago, F-75014 Paris, France

${ }^{14}$ Las Cumbres Observatory Global Telescope Network, 6740 Cortona Drive Suite 102, Goleta, CA 93117, USA

${ }^{15}$ Department of Physics, University of California, Santa Barbara, CA 93106-9530, USA

${ }^{16}$ NSF's Optical-Infrared Astronomy Research Lab, Tucson, AZ 85719, USA

${ }^{17}$ Department of Physics, Southern Connecticut State University, 501 Crescent Street, New Haven, CT 06515, USA

${ }^{18}$ Kavli Institute for Particle Astrophysics and Cosmology, Stanford University, Stanford, CA, USA

${ }_{19}$ NASA Ames Research Center, Moffett Field, CA 94035, USA

${ }^{20}$ Department of Physics, and Kavli Institute for Astrophysics and Space Research, Massachusetts Institute of Technology, Cambridge, MA 02139, USA

${ }^{21}$ Hungarian Astronomical Association, 1451 Budapest, Hungary

Received 2020 July 6; revised 2021 January 8; accepted 2021 February 15; published 2021 June 8

\begin{abstract}
We report the discovery and characterization of seven transiting exoplanets from the HATNet survey. The planets, which are hot Jupiters and Saturns transiting bright Sun-like stars, include: HAT-P-58b (with mass $M_{p}=0.37 M_{\mathrm{J}}$, radius $R_{p}=1.33 R_{\mathrm{J}}$, and orbital period $P=4.0138$ days), HAT-P-59b $\left(M_{p}=1.54 M_{\mathrm{J}}, R_{p}=1.12 R_{\mathrm{J}}, P=4.1420\right.$ days), HAT-P-60b $\left(M_{p}=0.57 M_{\mathrm{J}}, R_{p}=1.63 R_{\mathrm{J}}, P=4.7948\right.$ days), HAT-P-61b $\left(M_{p}=1.06 M_{\mathrm{J}}, R_{p}=0.90 R_{\mathrm{J}}\right.$, $P=1.9023$ days), HAT-P-62b $\left(M_{p}=0.76 M_{\mathrm{J}}, R_{p}=1.07 R_{\mathrm{J}}, P=2.6453\right.$ days $)$, HAT-P-63b $\left(M_{p}=0.61 M_{\mathrm{J}}\right.$, $R_{p}=1.12 R_{\mathrm{J}}, P=3.3777$ days), and HAT-P-64b $\left(M_{p}=0.58 M_{\mathrm{J}}, R_{p}=1.70 R_{\mathrm{J}}, P=4.0072\right.$ days $)$. The typical errors on these quantities are $0.06 M_{\mathrm{J}}, 0.03 R_{\mathrm{J}}$, and $0.2 \mathrm{~s}$, respectively. We also provide accurate stellar parameters for each of the host stars. With $V=9.710 \pm 0.050 \mathrm{mag}$, HAT-P-60 is an especially bright transiting planet host, and an excellent target for additional follow-up observations. With $R_{p}=1.703 \pm 0.070 R_{\mathrm{J}}$, HAT-P-64b is a highly inflated hot Jupiter around a star nearing the end of its main-sequence lifetime, and is among the largest known planets. Five of the seven systems have long-cadence observations by TESS which are included in the analysis. Of particular note is HAT-P-59 (TOI-1826.01) which is within the northern continuous viewing zone of the TESS mission, and HAT-P-60, which is the TESS candidate TOI-1580.01.
\end{abstract}

Unified Astronomy Thesaurus concepts: Exoplanets (498); Hot Jupiters (753); Exoplanet astronomy (486); Astronomical instrumentation (799)

Supporting material: machine-readable tables

\section{Introduction}

The Hungarian-made Automated Telescope Network (HATNet; Bakos et al. 2004) began initial operations in 2003, with the primary science goal of discovering and accurately

\footnotetext{
* Based on observations of the Hungarian-made Automated Telescope Network and observations obtained at the following observatories: W. M. Keck $\mathrm{k}$ Observatory, the $1.5 \mathrm{~m}$ and the $1.2 \mathrm{~m}$ telescopes at the Fred Lawrence Whipple Observatory of the Smithsonian Astrophysical Observatory, the Kitt Peak National Observatory, the $1.93 \mathrm{~m}$ telescope at Observatoire de HauteProvence, the Subaru Telescope of the National Astronomical Observatory of Japan, the Nordic Optical Telescope in the Spanish Observatorio del Roque de los Muchachos of the Intituto de Astrofísica de Canarias, and the Apache Point Observatory $3.5 \mathrm{~m}$ telescope.
}

characterizing transiting extrasolar planets (TEPs) around bright stars. It is one of four ongoing ground-based wide-field transiting planet surveys with more than 10 planet discoveries, the others being HATSouth (Bakos et al. 2013; although led by the same PI, this project is independent from the northern HATNet survey), SuperWASP (Pollacco et al. 2006), and KELT (Pepper et al. 2007).

HATNet consists of six $11 \mathrm{~cm}$ diameter telephoto lenses coupled to front-side-illuminated charged-coupled device (CCD) imagers, each in a separate mount and enclosure. Four of the units (called HAT-5, -6, -7, and -10) are located at Fred Lawrence Whipple Observatory (FLWO) in Arizona, while the other two (called HAT-8 and -9) are located on the roof of the 
Submillimeter Array service building at Mauna Kea Observatory (MKO) in Hawaii. The system has been fully operational in an autonomous fashion since 2004, and has remained nearly homogenous, with only a few changes to the instrumentation and observing procedures since that time. To date a total of 63 TEP discoveries have been published based on HATNet observations (the most recent being Zhou et al. 2019). Here we present the discovery of seven new TEP systems identified using HATNet, together with an accurate determination of the system parameters, including precise radial velocity (RV) observations used to measure the planetary masses. Before delving into a detailed discussion of these new discoveries, we first provide a brief update on the status of HATNet.

Since 2004 there have been four different combinations of CCD cameras and filters used by HATNet. The initial setup (until the summer of 2007) made use of Apogee AP10 $2 K \times 2 K$ CCDs and Cousins $I$-band filters. This provided a $8^{\circ} .2 \times 8^{\circ} .2$ field of view and a plate scale of $14^{\prime \prime}$ pixel $^{-1}$. In 2007 September we replaced the CCDs to Apogee U16m $4 K \times 4 K$ imagers, providing a larger field of view $\left(10^{\circ} .6 \times 10^{\circ} .6\right)$ and higher spatial resolution $\left(9^{\prime \prime}\right.$ pixel $\left.^{-1}\right)$. We also changed the filters to Bessel $R$-band to better match the peak quantum efficiency of the CCD, and a year later (in 2008 September), we changed the filters to Sloan $r$-band to have better overall response, and sharp wavelength boundaries. The majority of the HATNet survey was performed with this setup, i.e., the Apogee U16m $4 K \times 4 K$ imagers and the Sloan $r$-band filters. The most recent modification was in 2013 October, when the imager on HAT-7 at FLWO was changed to an FLI back-sideilluminated $2 K \times 2 K$ CCD device. The other units continue to use the Apogee U16m $4 K \times 4 K$ devices.

HATNet follows a point-and-stare mode of observation, where each unit is assigned a primary field (one of 838 discrete pointings which tile the full $4 \pi$ steradian celestial sphere), which it observes continuously over the night using 3-minute integrations, so long as the field is above $30^{\circ}$ elevation, and not too close to the Moon. A secondary field is also assigned to each instrument, which is observed when the primary field is not visible. In recent years we have adopted a strategy where all of the units are assigned the same primary and secondary fields, which we have found to significantly increase the sensitivity to small-radius planets. This is in contrast to our earlier mode of observing where different units are assigned different fields to maximize the sky coverage. The total time spent on a field varies significantly, from a minimum of 2000 observations, to as many as 40,000 observations collected (the median is 6000 observations). As of 2020 May, a total of 185 fields, corresponding to 148 unique pointing positions, ${ }^{22}$ and covering approximately $35 \%$ of the northern sky, have been observed, reduced, and searched for transiting planets. Some 9.3 million light curves have been generated from these images for 5.9 million stars ranging in brightness from $r \approx 9.5 \mathrm{mag}$ to $r=14.5$. The trend-filtered light curves reach a precision of $\sim 3$ mmag at cadence for the brightest sources. Based on these light curves a total of 2460 candidate transiting planets have been selected.

The majority of the candidates (approximately 2200 to date) have received at least some follow-up spectroscopic and/or photometric observations using a variety of facilities (e.g., Latham et al. 2009). Based on these observations, some 1950

\footnotetext{
22 We have revisited some sky positions with a different instrumental configuration leading to multiple "fields" for these positions.
}

of the candidates have been set aside as false positives or false alarms (i.e., cases where we suspect that the candidate transit signal detected in the HATNet light curve is spurious). In addition to those planets presented here, more than a dozen other planets have been confirmed, but have not yet been published. Some 250 candidates have received some follow-up, but require additional follow-up observations for confirmation and characterization.

The seven planets that are the focus of this paper are quite typical of the population of transiting planets that have been discovered thus far by HATNet. With planetary masses between $\quad 0.372 \pm 0.030 M_{\mathrm{J}} \quad$ (HAT-P-58b) and $1.540 \pm$ $0.067 M_{\mathrm{J}}$ (HAT-P-59b), orbital periods between 1.9023 days (HAT-P-61b) and 4.7948 days (HAT-P-60b), and host star masses between $0.925 \pm 0.023 M_{\odot}$ (HAT-P-63) and $1.298 \pm$ $0.021 M_{\odot}$ (HAT-P-64), these are all hot Jupiters transiting Sunlike stars. The host stars are all relatively bright, particularly HAT-P-60 at $V=9.710 \pm 0.050 \mathrm{mag}$, enabling the accurate determination of the orbital parameters, and planetary and stellar physical parameters, that we provide in this paper for each of these systems. The targets are also amenable to additional follow-up observations that may be carried out to characterize the orbital geometries (e.g., spin-orbit alignment measurements via the Rossiter-McLaughlin effect; Queloz et al. 2000) and planetary atmospheres (e.g., transmission spectroscopy; Charbonneau et al. 2002). The continued discovery and characterization of TEPs such as these increase the sample that may be used for statistical analysis of the population, which in turn provide insights into the physical processes involved in their formation and evolution. In fact, the planets reported here have already been included in a statistical analysis carried out by Hartman et al. (2016), which revealed observational evidence for the re-inflation of close-in giant planets.

In Section 2 we describe the observations collected to identify, confirm, and characterize the seven transiting planet systems presented here. The analysis carried out to measure the parameters of each system and to rule out blended stellar eclipsing binary false positive scenarios is described in Section 3. We discuss the results in Section 4.

\section{Observations}

\subsection{Photometric Detection}

Periodic transit events were first identified for all seven systems based on time-series photometric observations obtained with the HATNet wide-field photometric network (Bakos et al. 2004). The instruments and filters used, number of measurements collected and date range over which they were collected, observational cadence, and photometric precision achieved are all listed in Tables 1 and 2 for each of the seven systems. The raw HATNet images were reduced to light curves following Bakos et al. (2004), making use of aperture and image subtraction photometry routines based on the FITSH software package Pál (2012). Following Bakos et al. (2010) we filtered variations from the light curves that are correlated with a variety of auxiliary parameters, and we then applied the trendfiltering algorithm (TFA) of Kovács et al. (2005). The latter operates by fitting each light curve as a linear combination of "template" light curves (in our case these are light curves for a random sample of non-variable stars distributed across the image plane and in magnitude) and then subtracting the best-fit 
Table 1

Summary of Photometric Observations of HAT-P-58-HAT-P-61

\begin{tabular}{|c|c|c|c|c|c|}
\hline Instrument/Field ${ }^{\mathrm{a}}$ & Date(s) & \# Images $^{\mathrm{b}}$ & $\begin{array}{c}\text { Cadence }^{\mathrm{c}} \\
\text { (s) }\end{array}$ & Filter & $\begin{array}{r}\text { Precision } \\
\text { (mmag) }\end{array}$ \\
\hline \multicolumn{6}{|l|}{ HAT-P-58 } \\
\hline HAT-5/G093 & 2012 Sep-2013 Apr & 9254 & 213 & $r$ & 21.3 \\
\hline НАT-7/G093 & 2012 Sep & 238 & 213 & $r$ & 18.3 \\
\hline HAT-8/G093 & 2012 Jul-2013 Apr & 11,078 & 217 & $r$ & 14.8 \\
\hline FLWO $1.2 \mathrm{~m} /$ KeplerCam & 2014 Feb 1 & 157 & 48 & $i$ & 1.5 \\
\hline FLWO $1.2 \mathrm{~m} /$ KeplerCam & 2014 Feb 5 & 378 & 48 & $i$ & 1.5 \\
\hline FLWO $1.2 \mathrm{~m} /$ KeplerCam & $2014 \operatorname{Dec} 7$ & 188 & 51 & $i$ & 3.1 \\
\hline TESS/Sector 19 & 2019 Nov 29-2019 Dec 23 & 1117 & 1798 & $T$ & 1.1 \\
\hline \multicolumn{6}{|l|}{ HAT-P-59 } \\
\hline HAT-5/G081 & 2012 Oct-2012 Dec & 1963 & 213 & $r$ & 11.1 \\
\hline HAT-6/G081 & 2012 Sep-2012 Dec & 2500 & 214 & $r$ & 9.1 \\
\hline HAT-7/G081 & 2012 Jul-2012 Dec & 2340 & 213 & $r$ & 9.3 \\
\hline FLWO $1.2 \mathrm{~m} /$ KeplerCam & 2014 Mar 16 & 314 & 27 & $i$ & 2.1 \\
\hline FLWO $1.2 \mathrm{~m} /$ KeplerCam & 2014 May 13 & 642 & 26 & $i$ & 2.5 \\
\hline TESS/Sector 14 & 2019 Jul 18-2019 Aug 14 & 1233 & 1799 & $T$ & 0.75 \\
\hline TESS/Sector 15 & 2019 Aug 15-2019 Sep 8 & 821 & 1799 & $T$ & 0.72 \\
\hline TESS/Sector 16 & 2019 Sep $12-2019$ Oct 6 & 999 & 1799 & $T$ & 0.66 \\
\hline TESS/Sector 17 & 2019 Oct $8-31$ & 938 & 1799 & $T$ & 0.64 \\
\hline TESS/Sector 18 & 2019 Nov $4-27$ & 1036 & 1799 & $T$ & 0.63 \\
\hline TESS/Sector 20 & 2019 Dec 25-2020 Jan 20 & 1175 & 1799 & $T$ & 0.66 \\
\hline TESS/Sector 21 & 2020 Jan $23-2020$ Feb 18 & 1189 & 1799 & $T$ & 0.72 \\
\hline \multicolumn{6}{|l|}{ HAT-P-60 } \\
\hline HAT-7/G089 & 2009 Sep-2010 Mar & 5577 & 225 & $r$ & 4.4 \\
\hline FLWO $1.2 \mathrm{~m} /$ KeplerCam & 2013 Oct 20 & 873 & 25 & $z$ & 3.6 \\
\hline FLWO $1.2 \mathrm{~m} /$ KeplerCam & 2014 Sep 21 & 165 & 58 & $i$ & 1.5 \\
\hline FLWO $1.2 \mathrm{~m} /$ KeplerCam & 2014 Oct 10 & 280 & 59 & $i$ & 2.4 \\
\hline TESS/Sector 19 & 2019 Nov 28-2019 Dec 23 & 1145 & 1799 & $T$ & 1.1 \\
\hline
\end{tabular}

Notes.

${ }^{\text {a }}$ For HATNet data we list the HATNet unit and field name from which the observations are taken. HAT-5, $-6,-7$ and -10 are located at the Fred Lawrence Whipple Observatory in Arizona. HAT-8 and -9 are located on the roof of the Smithsonian Astrophysical Observatory Submillimeter Array hangar building at Mauna Kea Observatory in Hawaii. Each field corresponds to one of 838 fixed pointings used to cover the full $4 \pi$ celestial sphere. All data from a given HATNet field are reduced together, while detrending through external parameter decorrelation is done independently for each unique unit+field combination.

$\mathrm{b}$ Excluding outliers and other images that were not included when modeling the light curves.

${ }^{\mathrm{c}}$ The median time between consecutive images rounded to the nearest second. Due to factors such as weather, the day-night cycle, and guiding and focus corrections, the cadence is only approximately uniform over short timescales.

${ }^{\mathrm{d}}$ The rms of the residuals from the best-fit model.

model from the light curve being filtered. In our initial pass we apply the filtering in signal recovery mode, where we assume the light curve contains no astrophysical variations. We then use the box least-squares (BLS; Kovács et al. 2002) method to search the filtered light curves for periodic transits. Once recovered, we then re-apply the trend filtering, this time in signal-reconstruction mode, where we simultaneously fit to the light curve the linear filter and a periodic box-shaped transit model. This produces a filtered light curve without distorting the transit signal. The final trend-filtered photometric data for each system are shown phase-folded in Figure 1, and in Figures $7-12$ at the end of the paper, while the measurements are available in Table 4.

We used the VARTOOLS package (Hartman \& Bakos 2016) to search the residual HATNet light curves of each target for additional periodic transit signals using BLS, but do not find any significant signals attributable to additional transiting planets around these stars. For HAT-P-58 the highest peak in the BLS spectrum (in the residual light curve) is at $P=38.5$ days with a signal-to-pink noise ratio $\left(\mathrm{S} / \mathrm{N}_{\text {pink }}\right)$ of 5.5 (we 
Table 2

Summary of Photometric Observations of HAT-P-62-HAT-P-64

\begin{tabular}{|c|c|c|c|c|c|}
\hline Instrument/Field & Date(s) & \# Images & $\begin{array}{c}\text { Cadence } \\
\text { (s) }\end{array}$ & Filter & $\begin{array}{c}\text { Precision } \\
\text { (mmag) }\end{array}$ \\
\hline \multicolumn{6}{|l|}{ HAT-P-62 } \\
\hline HAT-5/G093 & 2012 Sep-2013 Apr & 9472 & 213 & $r$ & 15.1 \\
\hline HAT-7/G093 & 2012 Sep & 240 & 213 & $r$ & 13.7 \\
\hline HAT-8/G093 & 2012 Jul-2013 Apr & 11,093 & 217 & $r$ & 12.9 \\
\hline FLWO $1.2 \mathrm{~m} /$ KeplerCam & 2014 Dec 1 & 192 & 41 & $z$ & 2.1 \\
\hline FLWO $1.2 \mathrm{~m} /$ KeplerCam & $2014 \operatorname{Dec} 9$ & 376 & 41 & $i$ & 1.7 \\
\hline FLWO $1.2 \mathrm{~m} /$ KeplerCam & 2015 Jan 10 & 136 & 40 & $i$ & 2.1 \\
\hline FLWO 1.2 m/KeplerCam & 2015 Mar 4 & 363 & 39 & $i$ & 3.5 \\
\hline FLWO $1.2 \mathrm{~m} /$ KeplerCam & 2015 Sep 26 & 335 & 41 & $i$ & 2.1 \\
\hline \multicolumn{6}{|l|}{ HAT-P-63 } \\
\hline HAT-5/G384 & 2009 May-2009 Jun & 389 & 416 & $r$ & 12.4 \\
\hline НAT-9/G384 & 2009 May-2009 Sep & 2361 & 356 & $r$ & 9.6 \\
\hline FLWO $1.2 \mathrm{~m} /$ KeplerCam & 2013 Mar 13 & 111 & 86 & $i$ & 2.1 \\
\hline FLWO $1.2 \mathrm{~m} /$ KeplerCam & 2013 Mar 30 & 68 & 175 & $i$ & 1.5 \\
\hline FLWO $1.2 \mathrm{~m} /$ KeplerCam & 2013 Apr 16 & 157 & 86 & $i$ & 2.4 \\
\hline \multicolumn{6}{|l|}{ HAT-P-64 } \\
\hline HAT-6/G357 & 2009 Sep-2010 Mar & 3885 & 343 & $r$ & 14.1 \\
\hline HAT-8/G357 & 2009 Sep-2010 Mar & 9097 & 224 & $r$ & 14.6 \\
\hline FLWO 1.2 m/KeplerCam & 2011 Feb 2 & 93 & 105 & $i$ & 1.5 \\
\hline FLWO 1.2 m/KeplerCam & 2011 Oct 12 & 182 & 73 & $i$ & 2.3 \\
\hline TESS/Sector 5 & 2018 Nov 15-2018 Dec 11 & 1149 & 1799 & $T$ & 0.99 \\
\hline
\end{tabular}

Note.

a See footnotes to Table 1 for additional clarification on the contents of this table.

require $\mathrm{S} / \mathrm{N}_{\text {pink }}>7.0$ for detection) and a transit depth of $6.3 \mathrm{mmag}$. For HAT-P-59 we detect a signal at the sidereal frequency, which is presumably due to systematic errors in the photometry that are not fully removed through external parameter decorrelation (EPD) and TFA. The first harmonic of this same signal is also detected with the generalized LombScargle periodogram (GLS; Zechmeister \& Kürster 2009), and when it is filtered from the light curve using a Fourier series fit, we find no other significant transit signals with BLS. Altogether, we find the following peaks, significances, and transit depths in the residual light curves:

1. HAT-P-58, $P=38.5$ days, $\mathrm{S} / \mathrm{N}_{\text {pink }}=5.5,6.3 \mathrm{mmag}$;

2. HAT-P-59, $P=1.59$ days, $\mathrm{S} / \mathrm{N}_{\text {pink }}=6.0,2.3 \mathrm{mmag}$;

3. HAT-P-60, $P=2.48$ days, $\mathrm{S} / \mathrm{N}_{\text {pink }}=6.1,2.3 \mathrm{mmag}$;

4. HAT-P-61, $P=61.8$ days, $\mathrm{S} / \mathrm{N}_{\text {pink }}=5.2,2.7 \mathrm{mmag}$;

5. HAT-P-62, $P=0.146$ days, $\mathrm{S} / \mathrm{N}_{\text {pink }}=6.1,2.9 \mathrm{mmag}$;

6. HAT-P-63, $P=0.194$ days, $\mathrm{S} / \mathrm{N}_{\text {pink }}=6.0,8.3 \mathrm{mmag}$;

7. HAT-P-64, $P=0.438$ days, $\mathrm{S} / \mathrm{N}_{\text {pink }}=6.7,2.3 \mathrm{mmag}$.

We also used VARTOOLS to search the residual HATNet light curves for continuous periodic variability with the GLS periodogram. For HAT-P-58, HAT-P-60, and HAT-P-62HAT-P-64 we do not detect any periodic signals, and place 95\% confidence upper limits on the peak-to-peak amplitudes of such signals of $2.0 \mathrm{mmag}$ for HAT-P-58, $0.96 \mathrm{mmag}$ for HATP-60, 1.2 mmag for HAT-P-62, 3.9 mmag for HAT-P-63, and 2.0 mmag for HAT-P-64. For HAT-P-59 a strong signal with a period of $P=0.49976 \pm 0.00086$ days is detected with a peakto-peak amplitude of $16.6 \mathrm{mmag}$. Given the close proximity of the period to twice the sidereal frequency, we suspect that this signal is most likely due to systematic errors in the photometry that are not fully corrected through EPD and the TFA. After subtracting a Fourier series model from the light curve, the
GLS periodogram finds no additional signals present in the data, and we place a $95 \%$ confidence upper limit of $1.5 \mathrm{mmag}$ on the peak-to-peak amplitude of any such signals. For HAT-P61 we detect a possible signal with a period of $10.6 \pm 0.5$ days and with a formal false alarm probability of $0.16 \%$ and peak-topeak amplitude of $2.6 \mathrm{mmag}$. The GLS periodogram is shown in Figure 3. This may correspond to the photometric rotation period of the star, in which case the equatorial rotation velocity of $4.7 \mathrm{~km} \mathrm{~s}^{-1}$ is $2 \sigma$ larger than the spectroscopically measured projected rotation velocity of $v \sin i=3.69 \pm 0.50 \mathrm{~km} \mathrm{~s}^{-1}$.

\subsection{Spectroscopic Observations}

Spectroscopic observations of the TEP systems were carried out using the Tillinghast Reflector Echelle Spectrograph (TRES; Fúrész 2008) on the $1.5 \mathrm{~m}$ Tillinghast Reflector at FLWO, the SOPHIE spectrograph (Bouchy et al. 2009) on the Observatoire de Haute Provence (OHP) $1.93 \mathrm{~m}$ telescope in France, HIRES (Vogt et al. 1994) on the Keck-I $10 \mathrm{~m}$ at MKO together with its $\mathrm{I}_{2}$ absorption cell, the High Dispersion Spectrograph (HDS; Noguchi et al. 2002) and its $I_{2}$ cell (Kambe et al. 2002) on the Subaru $8 \mathrm{~m}$ at MKO, the Astrophysical Research Consortium Echelle Spectrometer (ARCES; Wang et al. 2003) on the ARC $3.5 \mathrm{~m}$ telescope at Apache Point Observatory (APO) in New Mexico, the fiber-fed Échelle Spectrograph (FIES) on the Nordic Optical Telescope (NOT) 2.5 m (Djupvik \& Andersen 2010) in La Palma, Spain, and the Network of Robotic Echelle Spectrographs (NRES; Siverd et al. 2018) on the LCOGT $1 \mathrm{~m}$ network. Table 3 summarizes the spectroscopic observations collected for each TEP system. Phased high-precision RV and bisector span (BS) measurements are shown for each system in Figures 1 and 7-12. The data are listed in Table 12 at the end of the paper. 
HAT-P-58 $P=4.01 d M_{P}=0.37 M_{\text {Jup }} R_{P}=1.33 R_{\text {Jup }} M_{S}=1.03 M_{\text {Sun }} R_{S}=1.53 R_{\text {Sun }}$
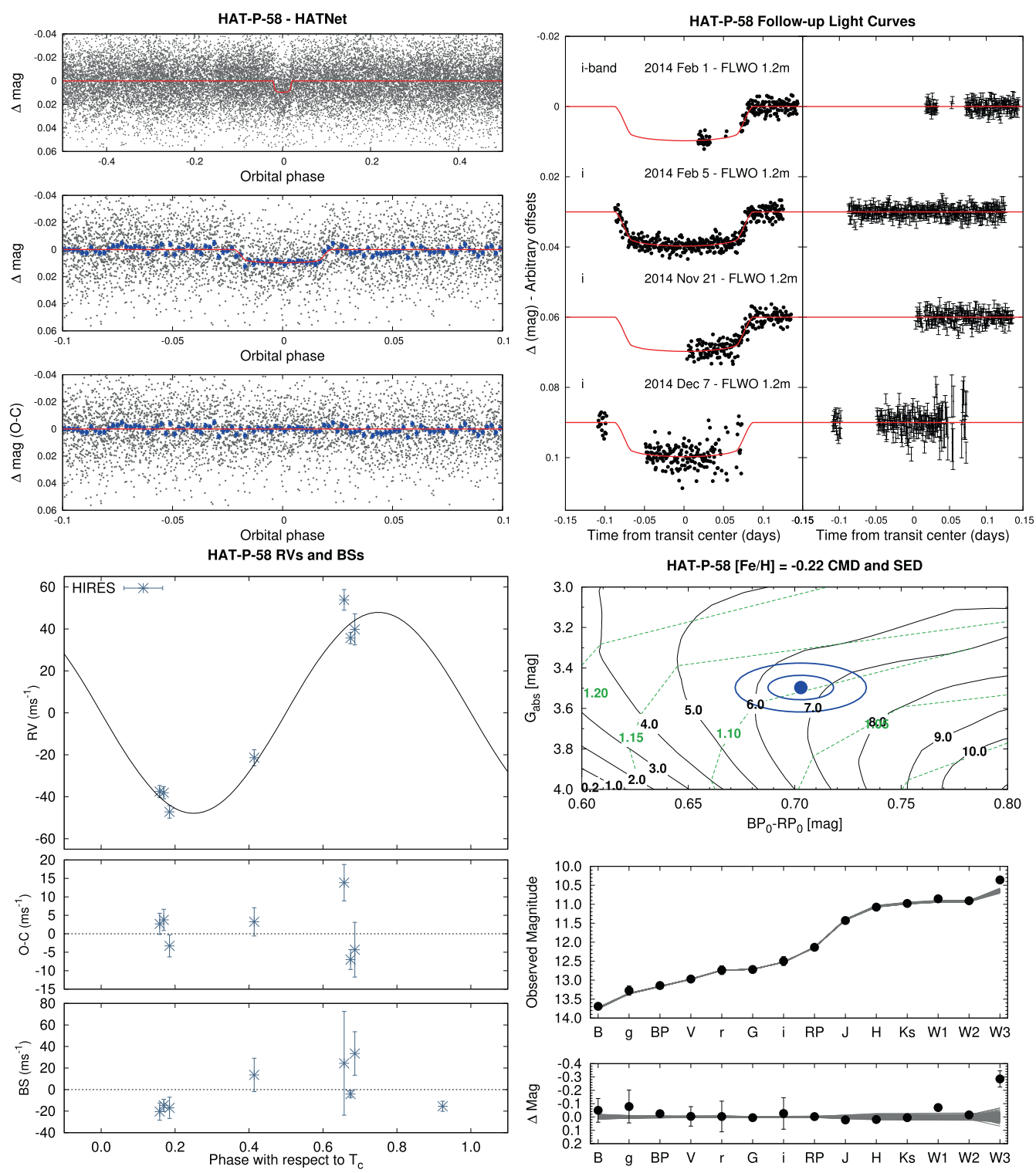

Figure 1. Observations used to confirm the transiting planet system HAT-P-58, excluding data from the NASA TESS mission which are shown in Figure 2. Top left: phasefolded unbinned HATNet light curve. The top panel shows the full light curve, the middle panel shows the light curve zoomed-in on the transit, and the bottom panel shows the residuals from the best-fit model zoomed-in on the transit. The solid lines show the model fits to the light curves. The dark filled circles show the light curves binned in phase with a bin size of 0.002 . Top right: unbinned follow-up transit light curves corrected for instrumental trends fitted simultaneously with the transit model, which is overplotted. The dates, filters, and instruments used are indicated. The residuals are shown on the right-hand side in the same order as the original light curves. The error bars represent the photon and background shot noise, plus the readout noise. Note that these uncertainties are scaled up in the fitting procedure to achieve a reduced $\chi^{2}$ of unity, but the uncertainties shown in the plot have not been scaled. Bottom left: high-precision radial velocities phased with respect to the mid-transit time. The instruments used are labeled in the plot. The top panel shows the phased measurements together with the best-fit model. The center-of-mass velocity has been subtracted. The second panel shows the velocity $O-C$ residuals. The error bars include the estimated jitter. The third panel shows the bisector spans. Bottom right: color-magnitude diagram and spectral energy distribution (shown here as magnitude vs. broadband filter rather than a more standard $F_{\lambda}$ vs. $\lambda$ to make it easier to judge the goodness of fit of the stellar model to the photometric observations). The top panel shows the absolute $G$ magnitude vs. the de-reddened $B P-R P$ color compared to theoretical isochrones (black lines) and stellar evolution tracks (green lines) from the PARSEC models interpolated at the best-estimate value for the metallicity of the host. The distance and reddening are as determined in our global analysis of the system (Section 3.3). The age of each isochrone is listed in black in gigayears, while the mass of each evolution track is listed in green in solar masses. The filled blue circles show the measured reddening- and distance-corrected values from Gaia DR2, while the blue lines indicate the $1 \sigma$ and $2 \sigma$ confidence regions, including the estimated systematic errors in the photometry. Note that the determination of the final age of the system is informed by other input parameters, such as the spectroscopic effective temperature, the broadband photometry in additional bandpasses and the stellar density from the light curves. The middle panel shows the magnitude vs. filter as measured via broadband photometry through the listed filters. Here we plot the observed magnitudes without correcting for distance or extinction. Overplotted are 200 model relations randomly selected from the Markov chain Monte Carlo posterior distribution produced through the global analysis (gray lines). The model makes use of the predicted absolute magnitudes in each bandpass from the PARSEC isochrones, the distance to the system (constrained largely via Gaia DR2), and extinction (constrained from the spectral energy distribution with a prior coming from the MWDUST 3D Galactic extinction model). The bottom panel shows the $O-C$ residuals from the best-fit model spectral energy distribution.

The TRES observations were reduced to spectra and crosscorrelated against synthetic stellar templates to measure the RVs and to estimate $T_{\text {eff }}, \log g$, and $v \sin i$. Here we followed the procedure of Buchhave et al. (2010), initially making use of a single order containing the gravity and temperature-sensitive $\mathrm{Mg} \mathrm{b}$ lines. Based on these observations we quickly ruled out 
Table 3

Summary of Spectroscopic Observations

\begin{tabular}{|c|c|c|c|c|c|c|}
\hline Instrument & UT Date(s) & \# Spec. & $\begin{array}{c}\text { Res. } \\
(\lambda / \Delta \lambda) / 1000\end{array}$ & $\mathrm{~S} / \mathrm{N}$ Range $^{\mathrm{a}}$ & $\begin{array}{c}\gamma_{\mathrm{RV}}^{\mathrm{b}} \\
\left(\mathrm{km} \mathrm{s}^{-1}\right)\end{array}$ & $\begin{array}{c}\text { RV Precision } \\
\left(\mathrm{m} \mathrm{s}^{-1}\right)\end{array}$ \\
\hline \multicolumn{7}{|l|}{ HAT-P-58 } \\
\hline FLWO $1.5 \mathrm{~m} /$ TRES & 2014 Jan $14-16$ & 2 & 44 & $16-19$ & -35.96 & 97 \\
\hline Keck-I/HIRES $+\mathrm{I}_{2}$ & 2014 Aug-Sep & 7 & 55 & $35-115$ & $\ldots$ & 8.2 \\
\hline Keck-I/HIRES & 2014 Aug 25 & 1 & 55 & 166 & $\cdots$ & $\cdots$ \\
\hline \multicolumn{7}{|l|}{ HAT-P-59 } \\
\hline FLWO $1.5 \mathrm{~m} /$ TRES & 2013 Oct-Nov & 13 & 44 & $13-25$ & -20.35 & 27 \\
\hline \multicolumn{7}{|l|}{ HAT-P-60 } \\
\hline FLWO 1.5 m/TRES & 2013 Feb-Oct & 13 & 44 & $20-61$ & 6.58 & 17 \\
\hline OHP 1.93 m/SOPHIE & 2013 Oct-Nov & 8 & 39 & $\ldots$ & 6.03 & 14 \\
\hline Keck-I/HIRES $+\mathrm{I}_{2}$ & 2013 Dec-2016 Jan & 8 & 55 & $140-196$ & $\cdots$ & 12 \\
\hline Keck-I/HIRES & 2015 Nov 29 & 1 & 55 & 306 & $\ldots$ & $\cdots$ \\
\hline LCO $1 \mathrm{~m}+\mathrm{ELP} / \mathrm{NRES}$ & 2019 Dec-2020 Jan & 12 & 53 & $32-65$ & 5.92 & 63 \\
\hline \multicolumn{6}{|l|}{ HAT-P-61 } & 57 \\
\hline FLWO 1.5 m/TRES & 2014 Sep-Nov & 18 & 44 & $12-22$ & 4.81 & 53 \\
\hline Keck-I/HIRES $+\mathrm{I}_{2}$ & 2015 Nov $27-29$ & 3 & 55 & $63-95$ & $\ldots$ & 9.3 \\
\hline Keck-I/HIRES & 2015 Nov 29 & 1 & 55 & 119 & $\cdots$ & $\cdots$ \\
\hline \multicolumn{7}{|l|}{ HAT-P-62 } \\
\hline FLWO 1.5 m/TRES & 2014 Jan-Nov & 15 & 44 & $15-25$ & 50.42 & 37 \\
\hline \multicolumn{7}{|l|}{ HAT-P-63 } \\
\hline FLWO $1.5 \mathrm{~m} /$ TRES $^{\mathrm{d}}$ & 2012 Apr 6-28 & 3 & 44 & $13-15$ & -68.92 & 33 \\
\hline APO 3.5 m/ARCES & 2012 Apr 30 & 1 & 31.5 & 18 & -69.57 & 500 \\
\hline Subaru 8 m/HDS & 2012 Sep 19 & 4 & 60 & $41-44$ & $\ldots$ & $\cdots$ \\
\hline Subaru $8 \mathrm{~m} / \mathrm{HDS}+\mathrm{I}_{2}$ & 2012 Sep $20-22$ & 12 & 60 & $37-55$ & $\ldots$ & 4.7 \\
\hline NOT 2.5 m/FIES & 2013 May 14 & 1 & 46 & 50 & -69.11 & 100 \\
\hline NOT $2.5 \mathrm{~m} / \mathrm{FIES}$ & 2013 May $15-17$ & 2 & 67 & $15-24$ & -69.045 & 66 \\
\hline OHP 1.93 m/SOPHIE & 2013 Jun $3-13$ & 7 & 39 & $\ldots$ & -69.60 & 23 \\
\hline \multicolumn{7}{|l|}{ HAT-P-64 } \\
\hline FLWO $1.5 \mathrm{~m} /$ TRES & 2010 Oct-2011 Jan & 2 & 44 & $25-28$ & 25.220 & 58 \\
\hline NOT 2.5 m/FIES & 2011 Oct $9-25$ & 4 & 67 & $44-54$ & 25.142 & 65 \\
\hline Keck-I/HIRES & 2011 Jan-Sep & 2 & 55 & $96-138$ & $\ldots$ & $\ldots$ \\
\hline Keck-I/HIRES $+\mathrm{I}_{2}$ & 2011-2012 Jan & 7 & 55 & $80-113$ & $\cdots$ & 22 \\
\hline OHP 1.93 m/SOPHIE & 2011 Dec 5-12 & 6 & 39 & $\ldots$ & 24.49 & 35 \\
\hline
\end{tabular}

Notes.

${ }^{a} \mathrm{~S} / \mathrm{N}$ per resolution element near $5180 \AA$ A. This was not reported for the OHP $1.93 \mathrm{~m} / \mathrm{SOPHIE}$ observations.

${ }^{\mathrm{b}}$ For high-precision RV observations included in the orbit determination this is the zero-point RV from the best-fit orbit. For other instruments it is the mean value. We do not provide this quantity for the Keck-I/HIRES observations, from which we have only measured relative RVs. Due to differences in the methods used to calibrate the RV zero-points of each instrument to an absolute system, instrumental differences in the reported systemic RV at the $\sim 1 \mathrm{~km} \mathrm{~s}^{-1}$ level can be expected. ${ }^{\mathrm{c}}$ For high-precision RV observations included in the orbit determination this is the scatter in the RV residuals from the best-fit orbit (which may include astrophysical jitter); for other instruments this is either an estimate of the precision (not including jitter), or the measured standard deviation. We do not provide this quantity for the $\mathrm{I}_{2}$-free templates obtained with Keck-I/HIRES or Subaru/HDS.

${ }^{\mathrm{d}}$ One of the TRES spectra of HAT-P-63 was low S/N and did not permit high-precision RVs, so only two of the TRES RVs of this object are included in the analysis.

common false positive scenarios, such as transiting $\mathrm{M}$ dwarf stars, or blends between giant stars and pairs of eclipsing dwarf stars. For HAT-P-59 through HAT-P-63 the initial TRES RVs exhibited low-amplitude variations consistent with planetary mass companions, so we continued to collect spectroscopic observations with TRES for these objects with the aim of confirming them as TEP systems, measuring the masses of the planets, and providing high-precision stellar atmospheric parameters, including the stellar metallicities. For this work high-precision RVs and spectral line BSs were determined based on a multi-order analysis of the spectra (e.g., Bieryla et al. 2014), while the atmospheric parameters were determined using the Stellar Parameter Classification (SPC; Buchhave et al. 2012) method. For HAT-P-58 and HAT-P-64 the TRES observations were used solely for reconnaissance and were not included in the analysis described in Section 3.3.

The SOPHIE observations of HAT-P-59, HAT-P-60, HATP-63, and HAT-P-64 were reduced to RVs and BSs following Boisse et al. (2013). In all cases the RVs show variations consistent with planetary mass companions, and with the variations seen using other spectrographs.

The HIRES observations of HAT-P-58, HAT-P-60, HAT-P61, and HAT-P-64 were reduced to relative RVs following the method of Butler et al. (1996), and to BSs following Torres et al. (2007). We also measured Ca II $\mathrm{H}$ and $\mathrm{K}$ chromospheric residual emission indices (the so-called $S$ and $\log _{10} R_{\mathrm{HK}}^{\prime}$ indices) 
Table 4

Light-curve Data for HAT-P-58-HAT-P-64

\begin{tabular}{|c|c|c|c|c|c|c|}
\hline Object $^{\mathrm{a}}$ & $\begin{array}{l}\text { BJD }_{\text {TDB }}{ }^{\mathrm{b}} \\
(2,400,000+)\end{array}$ & $\mathrm{Mag}^{\mathrm{c}}$ & $\sigma_{\mathrm{Mag}}$ & $\operatorname{Mag}\left(\right.$ orig) ${ }^{d}$ & Filter & Instrument \\
\hline HAT-P-58 & 56239.13511 & 0.00565 & 0.01008 & $\ldots$ & $r$ & HATNet \\
\hline HAT-P-58 & 56235.12147 & -0.01055 & 0.01209 & $\ldots$ & $r$ & HATNet \\
\hline HAT-P-58 & 56243.14926 & -0.00973 & 0.01036 & $\ldots$ & $r$ & HATNet \\
\hline HAT-P-58 & 56194.98348 & 0.03111 & 0.01042 & $\ldots$ & $r$ & HATNet \\
\hline HAT-P-58 & 56211.03898 & -0.00313 & 0.01037 & $\ldots$ & $r$ & HATNet \\
\hline HAT-P-58 & 56198.99820 & -0.02555 & 0.01558 & $\ldots$ & $r$ & HATNet \\
\hline HAT-P-58 & 56383.63531 & 0.01115 & 0.01428 & $\ldots$ & $r$ & HATNet \\
\hline
\end{tabular}

Notes.

${ }^{\mathrm{a}}$ Either HAT-P-58, HAT-P-59, HAT-P-60, HAT-P-61, HAT-P-62, HAT-P-63, or HAT-P-64.

b Barycentric Julian date on the dynamical time system, including the correction for leap seconds.

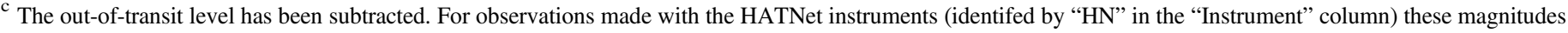

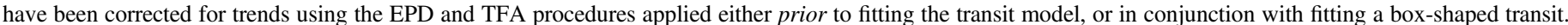

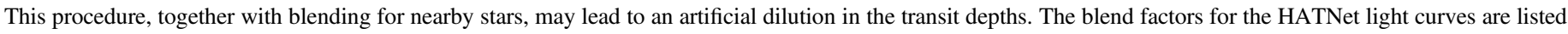

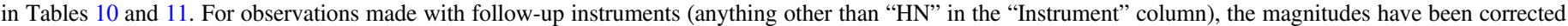

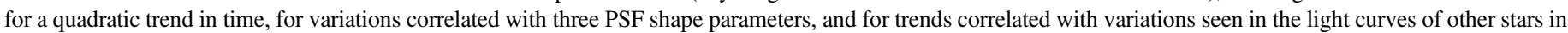
the field (the TFA method) fit simultaneously with the transit.

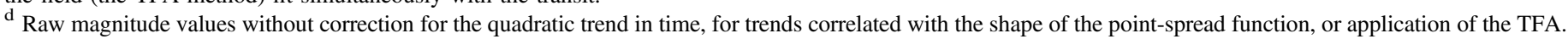
These are only reported for the follow-up observations.

(This table is available in its entirety in machine-readable form.)

following Isaacson \& Fischer (2010) and Noyes et al. (1984). For HAT-P-64 we measured stellar atmospheric parameters from the $\mathrm{I}_{2}$-free template spectra using the SPC.

The HDS observations of HAT-P-63 were reduced to relative RVs following Sato et al. $(2002,2012)$ and to BSs following Torres et al. (2007). The RVs are seen to vary in phase with the photometric ephemeris of the TEP, and are consistent with the variations seen with the TRES and SOPHIE spectrographs for this system.

The ARCES spectrum of HAT-P-63 was reduced following Hartman et al. (2015) and Buchhave et al. (2012) and was used for reconnaissance. The RV and atmospheric parameters of HAT-P-63 determined from this spectrum are consistent with the results from TRES.

The FIES spectra of HAT-P-63 and HAT-P-64 were reduced following Buchhave et al. (2010). For HAT-P-63 the first spectrum was obtained using the medium-resolution fiber, while the other spectra were obtained with the high-resolution fiber. For HAT-P-64 all four spectra were obtained with the high-resolution fiber. While the spectra were intended to be used for measuring the masses of the planetary companions, the resulting $\mathrm{RV}$ precision was insufficient for this purpose, given the small number of observations obtained. We therefore do not include these measurements in our analyses of HAT-P-63 or HAT-P-64.

NRES spectra of HAT-P-60 were collected from the McDonalds Observatory and Wise Observatory LCOGT $1 \mathrm{~m}$ facilities. We obtained 22 useful spectra with an $\mathrm{S} / \mathrm{N}$ between 32 and 65 , measured at $\sim 5150 \AA$. The exposure time for all spectra was $1800 \mathrm{~s}$. In order to obtain the wavelength-calibrated spectra and extract high-precision RVs, we adapted the CERES pipeline (Brahm et al. 2017). We limited the order extraction to the central 50 orders, covering the wavelength range from 4194 to $7445 \AA$.

\subsection{Ground-based Photometric Follow-up Observations}

In order to determine the physical parameters of each TEP system, we conducted follow-up photometric time-series observations of each object using KeplerCam on the $1.2 \mathrm{~m}$ telescope at FLWO. These observations are summarized in Tables 1 and 2, where we list the dates of the observed transit events, the number of images collected for each event, the cadence of the observations, the filters used, and the per-point photometric precision achieved. The images were reduced to light curves following Bakos et al. (2010), which are plotted in Figures 1 and 7-12. The data are provided in Table 4.

\subsection{TESS Space-based Photometry}

Five of the seven planetary systems presented here were observed by the NASA TESS mission (Ricker et al. 2015), as summarized in Tables 1 and 2. Of particular note is HAT-P-59 which is located in the northern TESS continuous viewing zone, and had data from Sectors 14, 15, 16, 17, 18, 20, and 21 that we included in the analysis. We were not able to extract useful photometry for this system from the Sector 19 observations. The two systems that did not have TESS observations were either too close to the ecliptic plane (HAT-P-63), or located only on the edge of a CCD in Sector 19, with no useful data collected (HAT-P-62).

We note that HAT-P-59b and HAT-P-60b have both been independently identified as candidate transiting planets based on the TESS observations. HAT-P-59b (a.k.a. TOI-1826.01) is listed as a community-identified candidate on ExoFOP-TESS, while HAT-P-60b (a.k.a. TOI-1580.01) is listed as a candidate identified by the MIT quick-look pipeline. All of the systems presented here were detected and confirmed as planets by the HATNet team prior to the launch of the TESS mission.

The five systems with TESS observations were all observed in long-cadence mode, and we extracted simple aperture photometry for them from the TESS Full-Frame Image (FFI) 
TESS Full Un-phased Light Curve of HAT-P-58
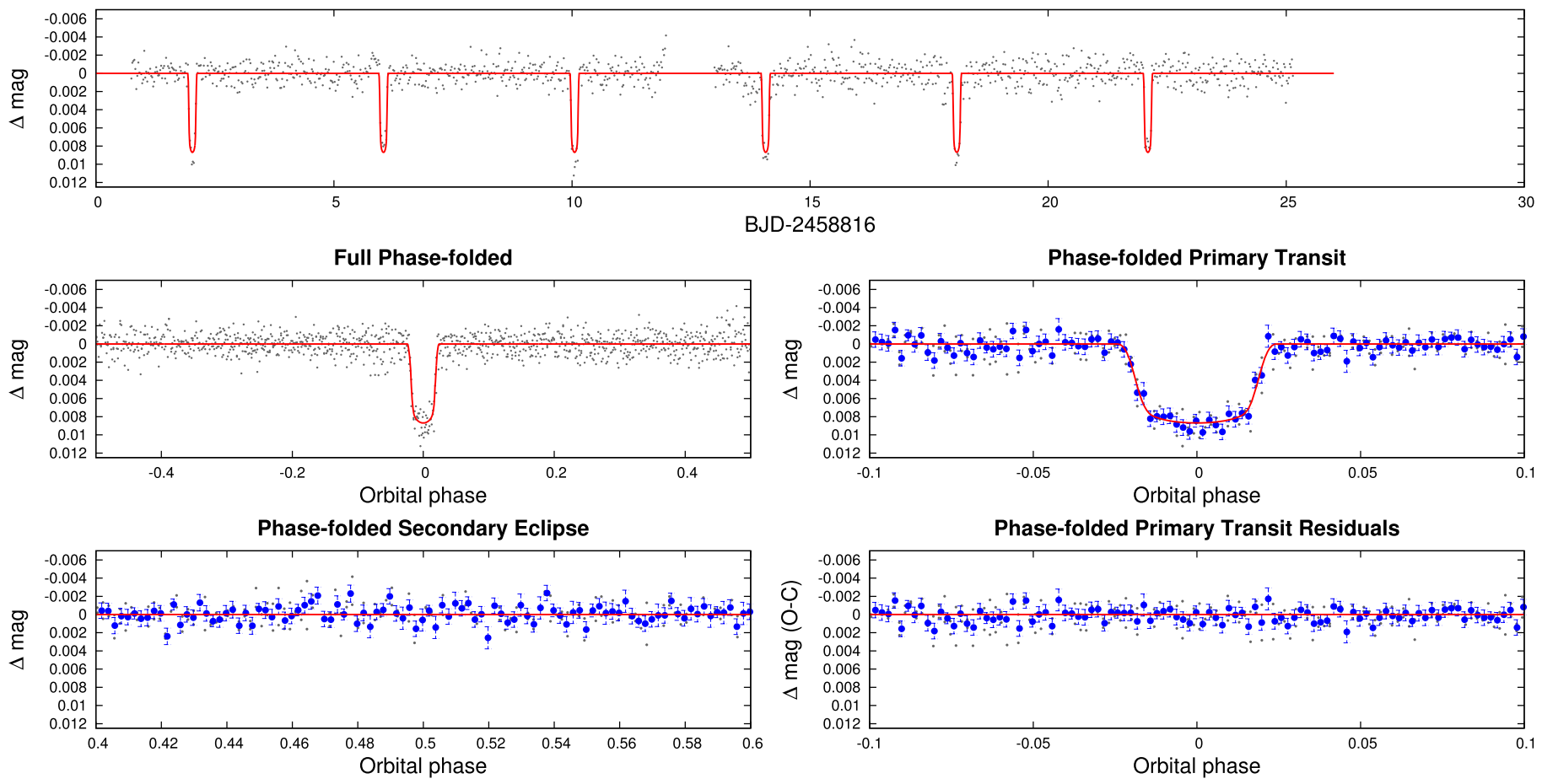

Figure 2. TESS long-cadence light curve for HAT-P-58. We show the full unphased light curve as a function of time (top), the full phase-folded light curve (middle left), the phase-folded light curve zoomed-in on the planetary transit (middle right), the phase-folded light curve zoomed-in on the secondary eclipse (bottom left), and the residuals from the best-fit model, phase-folded and zoomed-in on the planetary transit (bottom right). The solid line in each panel shows the model fit to the light curve, accounting for the 30 minute integrations. The dark filled circles show the light curve binned in phase with a bin size of 0.002 . Other observations included in our analysis of this system are shown in Figure 1.

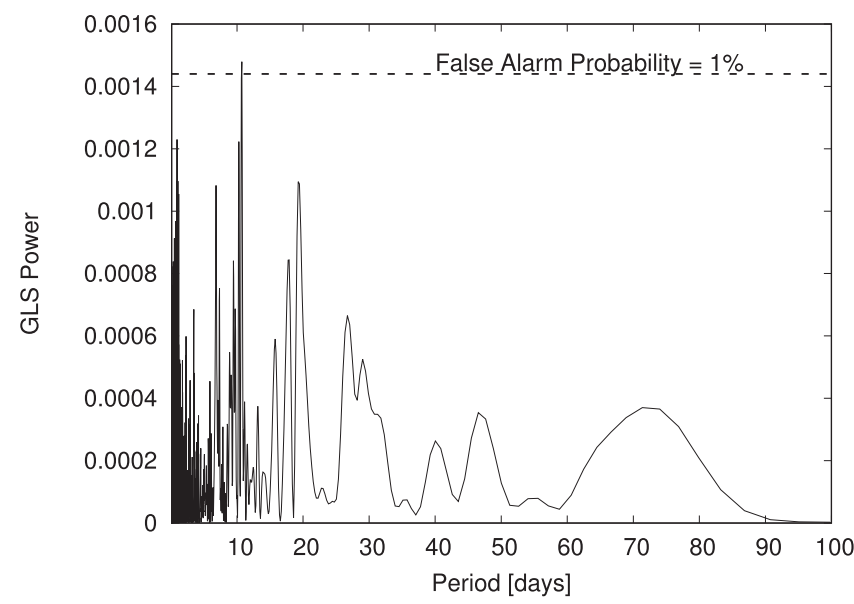

Figure 3. Generalized Lomb-Scargle periodogram of the HATNet observations of HAT-P-61 showing the possible detection of a $P=10.6$ day periodic signal in the light curve of this star.

data using the Lightkurve tool (Lightkurve Collaboration et al. 2018). Here we made use of the TESSCut API (Brasseur et al. 2019) to download $10 \times 10$ pixel FFI cutouts around each source, and made use of the automated mask routine in Lightkurve to generate the apertures using a threshold of 3.0, and to generate the background regions using a threshold of 0.001. We then used VARTOOLS (Hartman \& Bakos 2016) to apply a moving median filter to remove large systematic variations from the light curves. This was done by first manually removing regions from the light curves with excessive systematic behavior, then masking the transits and performing a median filter with a 0.5 day window. We then performed a monotonic spline interpolation over the masked regions of the light curves to estimate the systematic corrections to apply to the in-transit portions of the data. Note that the procedures above will likely erase the rotation induced and other long-term variation of the stars. The resulting light curves are shown, together with the best-fit models, in Figures 2 and 13-16. These data are also made available in Table 4.

As for the HATNet observations, we used the VARTOOLS package to search the residual TESS light curves of each target for additional periodic transit signals using BLS, and for additional sinusoidal periodic signals using the GLS periodogram. Table 5 gives the ephemeris information and significance for the top peak in the BLS spectrum of the TESS residuals for each system. None of the systems shows strong evidence for additional periodic transit signals. In a few cases (HAT-P-58 and HAT-P-59) there is marginal evidence for signals with $\mathrm{S} / \mathrm{N}_{\text {pink }}>7$ (see Hartman \& Bakos 2016 for a definition of this measure as used in VARTOOLS), though these are likely false alarms, and future observations by TESS in its extended mission should confirm or refute these. None of the systems shows evidence for a continuous periodic variation detected by the GLS periodogram, though any such variations would likely be removed by the median-filtering procedure that we applied to the light curves.

\subsection{Speckle Imaging Observations}

In order to detect nearby stellar companions which may be diluting the transit signals, we obtained high spatial resolution speckle imaging observations of all seven systems. For HAT-P58-HAT-P-62 and HAT-P-64 we used the Differential Speckle 

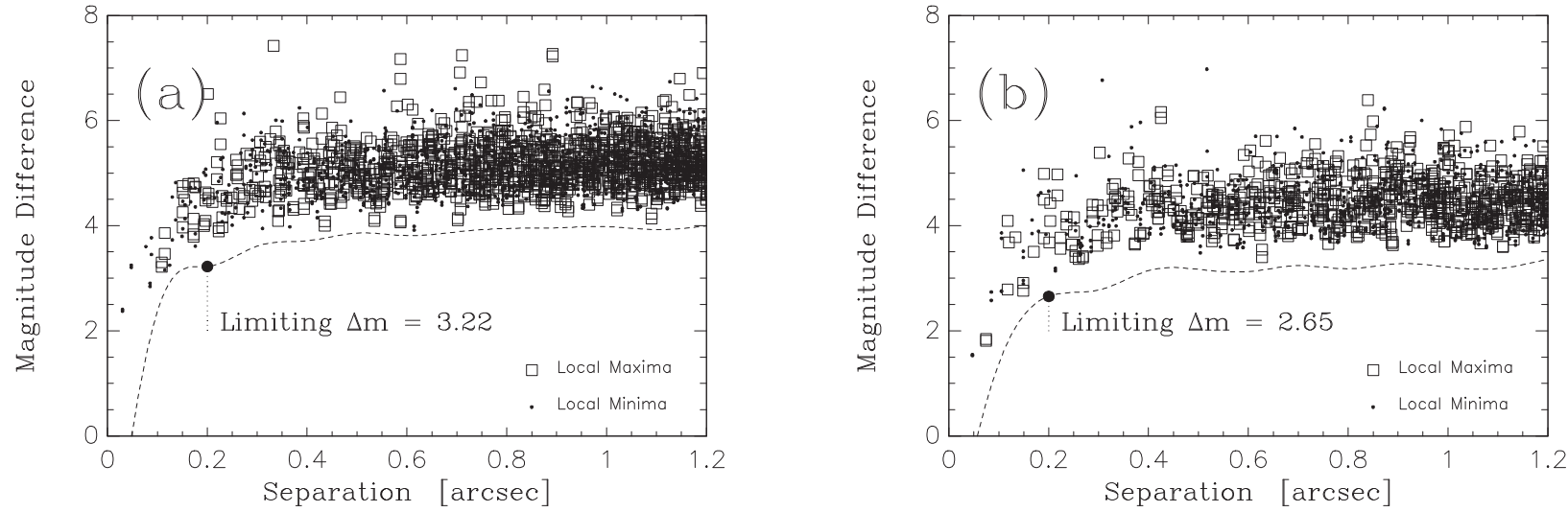

Figure 4. Limits on the relative magnitude of a resolved companion to HAT-P-58 as a function of angular separation based on speckle imaging observations from WIYN $3.5 \mathrm{~m} /$ DSSI. The left panel shows the limits for the $692 \mathrm{~nm}$ filter; the right shows limits for the $880 \mathrm{~nm}$ filter.
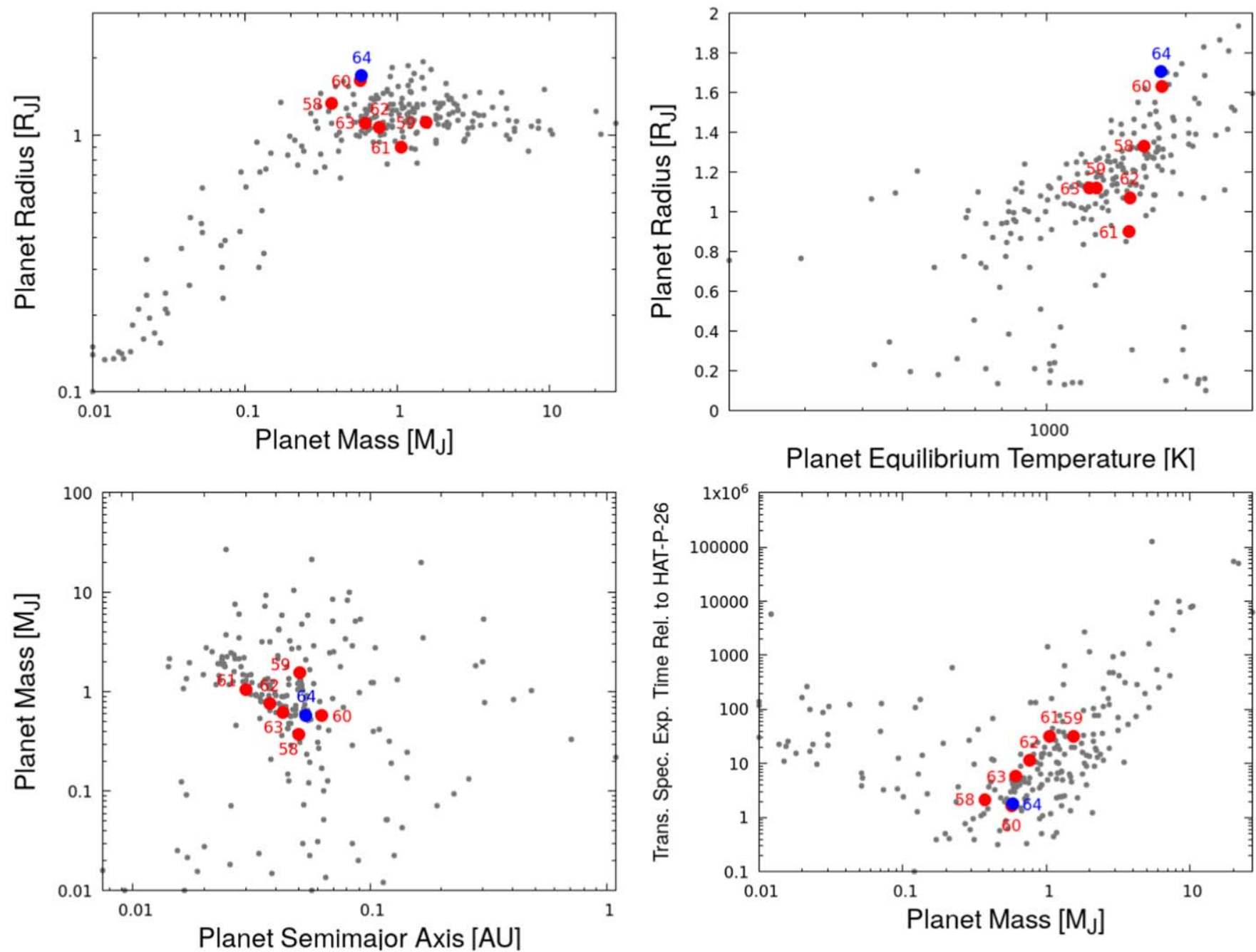

Figure 5. Comparison of the seven transiting planet systems presented here to the known sample of transiting planets with masses and radii both measured to better than 10\% precision (NASA Exoplanet Archive, accessed 2020 October 9). In each panel the known planets are shown with light gray circles, while the new planets are shown as red circles (for systems with masses and radii measured to better than $10 \%$ precision) and blue circles (for HAT-P-64b which has a lower precision mass measurement), and are labeled by the HAT planet system number. Top left: a mass-radius diagram showing that the newly discovered planets are all gas giants. Top right: planet radius vs. approximate equilibrium temperature (assuming zero albedo and full redistribution of heat). HAT-P-58b, HAT-P-60b, and HAT-P-64b are hot, inflated planets. Bottom left: planet mass vs. semimajor axis. The new planets are all close-in hot Jupiters, several of which fall along the edge of the planet distribution defining the so-called hot-Neptune desert. Bottom right: the approximate expected observing time required to obtain a planetary atmosphere transmission spectrum at fixed signal-to-noise relative to the hot-Neptune HAT-P-26b (observed by Wakeford et al. 2017). The transmission spectrum signal is assumed to scale as $H R_{p} / R_{\star}^{2}$, where $H$ is the atmospheric scale height, which we calculate assuming a mean molecular weight of $2.3 \mathrm{amu}$. We estimate the noise assuming observations in the $K_{S^{-}}$ band. The two inflated planets HAT-P-60b and HAT-P-64b have large atmospheric scale heights and will require relatively short observations to achieve high-S/N transmission spectra. 
Isochrone Ages: 1, 2, 4, 6, 8, 10 Gyr (left to right)

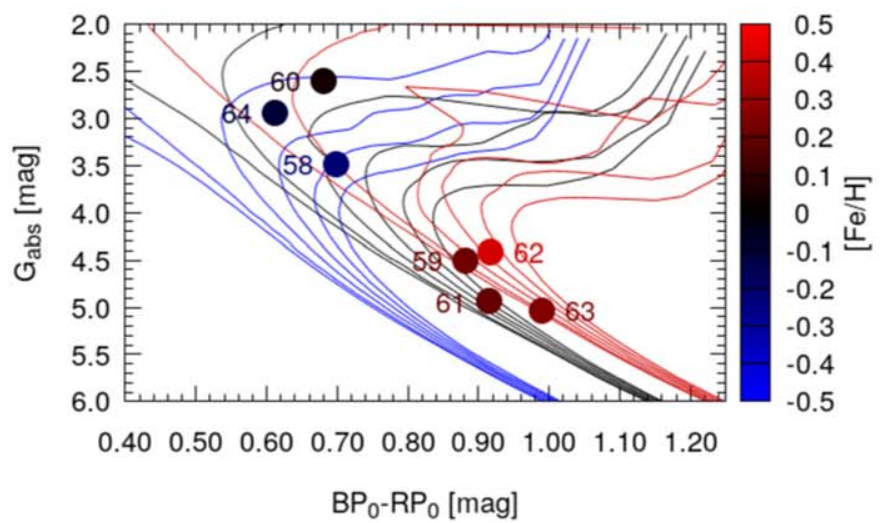

Isochrone Ages: 1, 2, 4, 6, 8, 10 Gyr (left to right)

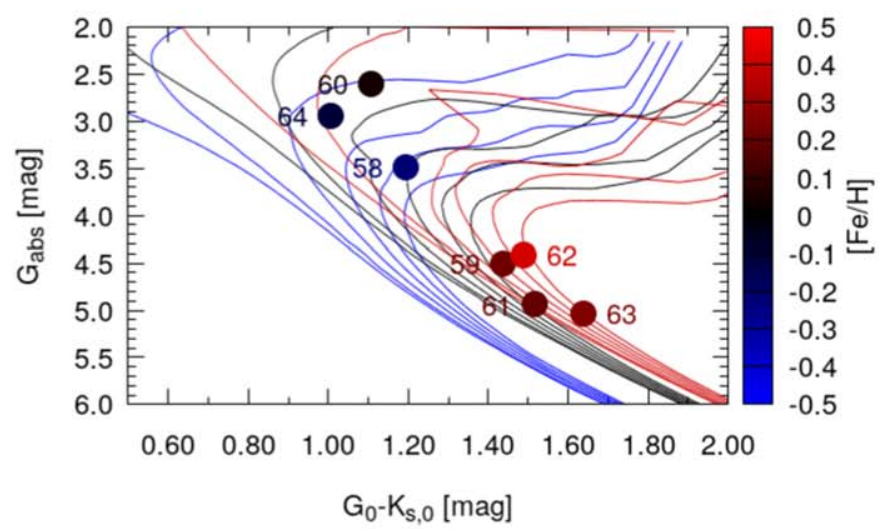

Figure 6. Location of all seven planet hosting stars on an absolute $G$ vs. de-reddened $B P-R P$ color-magnitude diagram (left), and an absolute $G$ vs. de-reddened $G-K_{S}$ color-magnitude diagram (right). We overlay the PARSEC isochrones showing models for metallicities of $[\mathrm{Fe} / \mathrm{H}]=-0.5,0$, and +0.5 dex $($ with the metallicity indicated by the color of the lines), and ages of 1.0, 2.0, 4.0, 6.0, 8.0, and 10.0 Gyr (with younger isochrones on the left and older ones on the right). The color of each point indicates the derived best-estimate metallicity for each host star, as indicated in Tables 8 and 9 (note that this may differ from the spectroscopically determined value for each star). The point sizes are comparable to the color uncertainties. Based on this plot we see that HAT-P-58, HAT-P-60, and HAT-P-64 are all stars at the main-sequence turn-off. These three stars host the highest equilibrium temperature, and most inflated planets among our sample.

Survey Instrument (DSSI; Horch et al. 2009, 2011, 2012; Howell et al. 2011), while for HAT-P-63 we used the newer NN-explore Exoplanet Stellar Speckle Imager (NESSI; Scott et al. 2018). Both instruments were used with the WIYN $3.5 \mathrm{~m}$ telescope $^{23}$ at Kitt Peak National Observatory in Arizona.

The DSSI observations were gathered between the nights of UT 2015 September 26 and UT 2015 October 3. A dichroic beamsplitter is used to obtain simultaneous imaging through $692 \mathrm{~nm}$ and $880 \mathrm{~nm}$ filters. Each observation consists of a sequence of $100040 \mathrm{~ms}$ exposures readout on $128 \times 128$ pixel $(2$ !" $8 \times 2$." 8$)$ subframes, which are reduced to reconstructed images following Horch et al. (2011). These images are searched for companions, and when none are detected, $5 \sigma$ lower limits on the differential magnitude between a putative companion and the primary star are determined as a function of angular separation as described in Horch et al. (2011).

The NESSI observation was gathered on the night of UT 2017 September 7, in this case using a dichroic beamsplitter to image at 562 and $832 \mathrm{~nm}$. The observing mode and reduction method are similar to those used for DSSI, and have been detailed in Scott et al. (2018). In this case the $256 \times 256$ pixel subframe has a field of view of 4 ". $6 \times 4$ ". 6 .

For HAT-P-60 we obtained a single observation, while for the other six objects we obtained five observations apiece. In all cases no companions are detected within 1. "2, and we place limits on the differential magnitudes in the blue and red filters as shown in Figures 4 and 17-22. We find limiting magnitude differences at $\sim 0$ !" 2 of

1. HAT-P-58- $\Delta m_{692}>3.22$ and $\Delta m_{880}>2.65$

2. HAT-P-59- $\Delta m_{692}>3.14$ and $\Delta m_{880}>2.74$

3. HAT-P-60- $\Delta m_{692}>4.04$ and $\Delta m_{880}>3.41$

4. HAT-P-61- $\Delta m_{692}>2.85$ and $\Delta m_{880}>2.62$

5. HAT-P-62- $\Delta m_{692}>3.16$ and $\Delta m_{880}>2.81$

6. HAT-P-63- $\Delta m_{562}>3.82$ and $\Delta m_{832}>3.55$

7. HAT-P-64- $\Delta m_{692}>2.60$ and $\Delta m_{880}>2.80$.

\footnotetext{
${ }^{23}$ The WIYN Observatory is a joint facility of the University of WisconsinMadison, Indiana University, the National Optical Astronomy Observatory, and the University of Missouri.
}

In addition to the companion limits based on the WIYN $3.5 \mathrm{~m} /$ DSSI observations, we also queried the UCAC 4 catalog (Zacharias et al. 2013) for neighbors within $20^{\prime \prime}$ and the Gaia DR2 catalog (Gaia Collaboration et al. 2018) for neighbors within $10^{\prime \prime}$ that may dilute either the HATNet or KeplerCam photometry. We find that HAT-P-60, HAT-P-62, and HAT-P-64 have fainter neighbors in Gaia DR2, while only the neighbor for HAT-P-62 is also detected in UCAC 4. The neighbors have separations and $G$-band magnitude differences as follows:

1. HAT-P-60-9". 088 southeast, $\Delta G=10.79 \mathrm{mag}$

2. HAT-P-62-5".565 northwest, $\Delta G=2.10 \mathrm{mag}, \Delta V=$ $2.18 \mathrm{mag}$

3. HAT-P-64-2". 510 northwest, $\Delta G=6.38 \mathrm{mag}$.

Based on the Gaia DR2 parallaxes the neighbors to HAT-P-60 and HAT-P-62 are background objects that are not physically associated with the planet hosts. No parallax, proper motion, or color information is available for the neighbor to HAT-P-64. This neighbor is at a projected separation of 1667 au from the planet host, if it is physically associated. The neighbors to HAT-P-60 and HAT-P-64 are too faint to significantly affect the photometry and the resulting planet and stellar parameters, and can be ruled out as the source of the detected transit signals. We do account for the neighbor to HAT-P-62 $(\Delta G=2.10 \mathrm{mag})$ in the analysis of this system as described in Section 3.3.

\section{Analysis}

We analyzed the photometric and spectroscopic observations of HAT-P-58-HAT-P-64 to determine the parameters of each system. The analysis followed the methods discussed in detail most recently by Hartman et al. (2019). Here we give a brief summary of the procedure.

\subsection{Properties of the Parent Star}

High-precision atmospheric parameters, including the effective surface temperature $T_{\text {eff } t}$, the surface gravity $\log g$, the metallicity $[\mathrm{Fe} / \mathrm{H}]$, and the projected rotational velocity $v \sin i$, were determined by applying the SPC to our high-resolution 

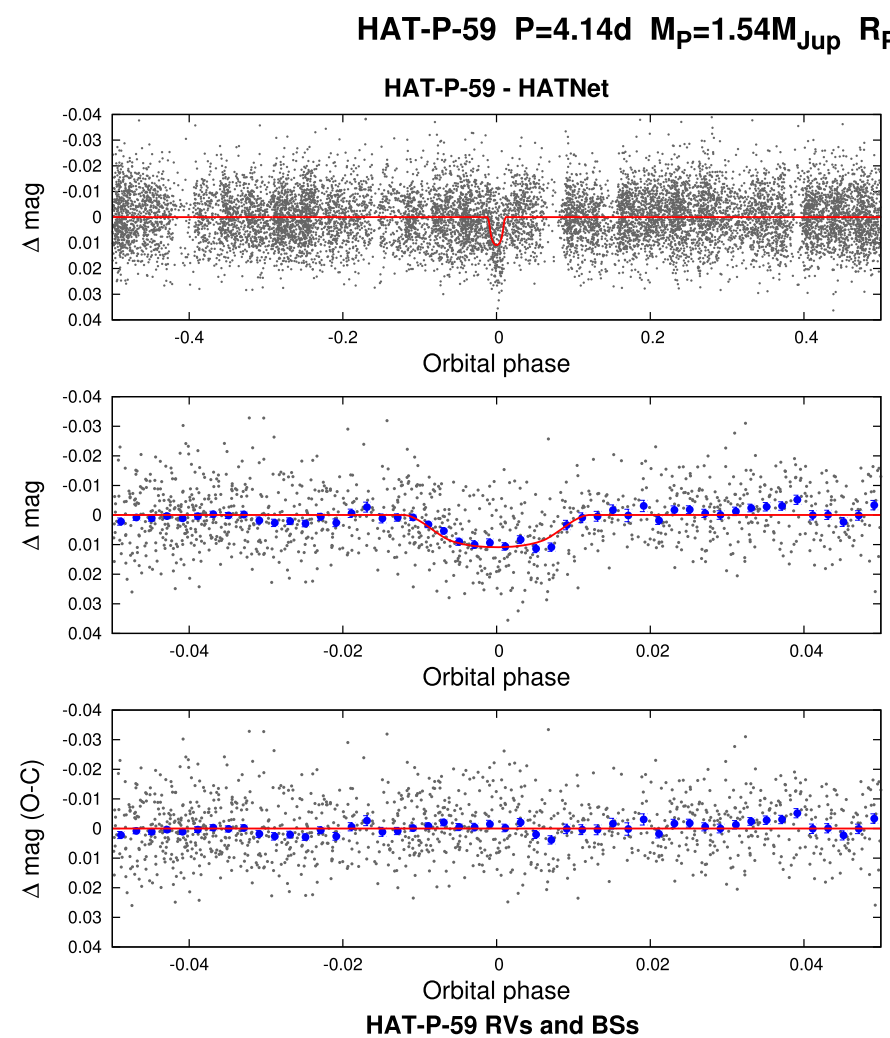

$P_{P}=1.12 R_{\text {Jup }} M_{S}=1.01 M_{\text {Sun }} R_{S}=1.10 R_{\text {Sun }}$
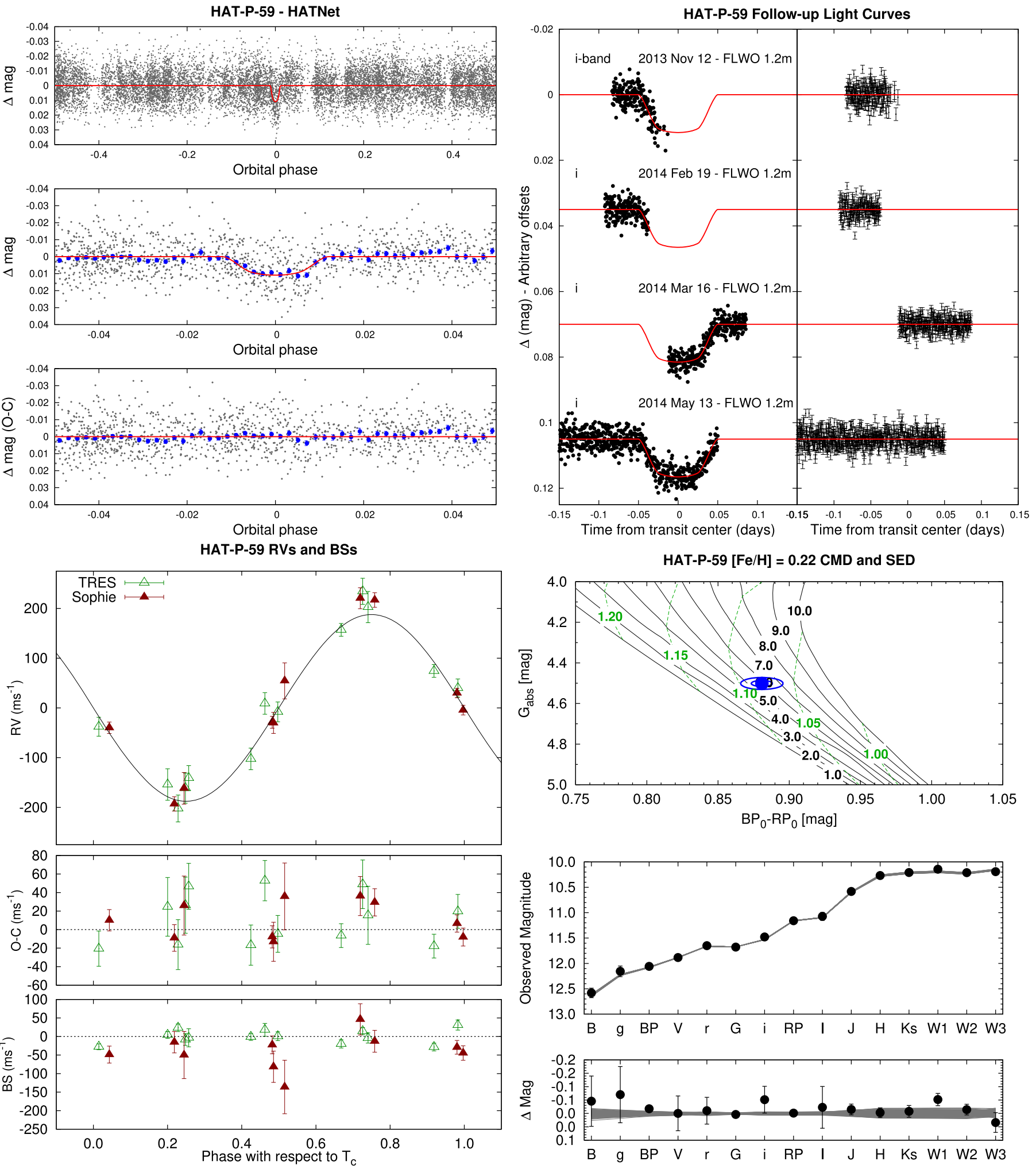

Figure 7. Observations of HAT-P-59 together with our best-fit model. See Figure 1 for a more detailed caption. The TESS light curve for this system is shown in Figure 13.

spectra. For HAT-P-58 through HAT-P-63 this analysis was performed on the TRES spectra, while for HAT-P-64 we made use of the Keck-I/HIRES $\mathrm{I}_{2}$-free template spectra. The analysis was performed separately on each spectrum and we took the weighted average of the results over all spectra obtained for each target. Here we assumed minimum uncertainties of 

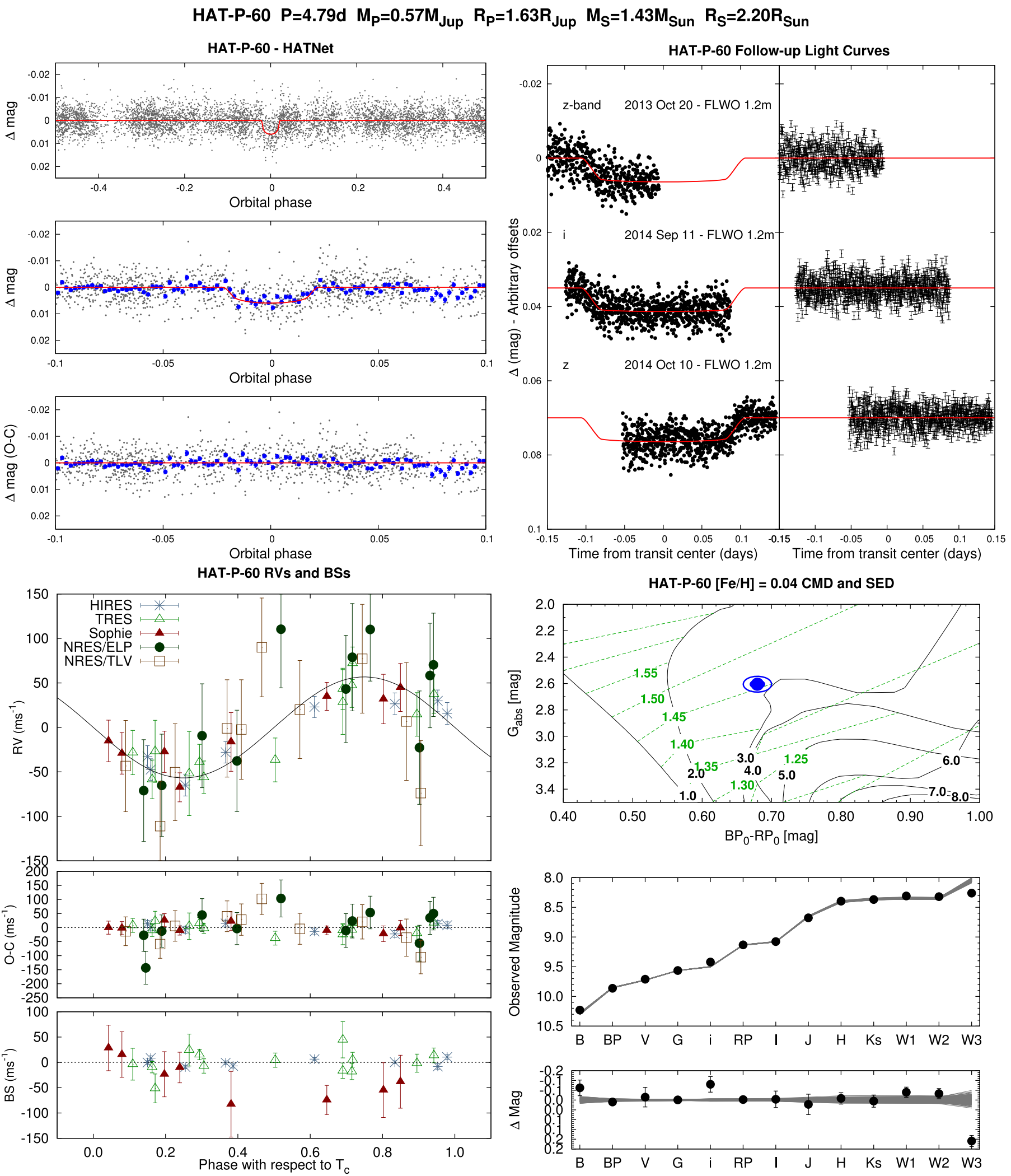

Figure 8. Observations of HAT-P-60 together with our best-fit model. See Figure 1 for a more detailed caption. The TESS light curve for this system is shown in Figure 14.

$50 \mathrm{~K}$ on $T_{\text {eff }}, 0.10 \mathrm{dex}$ on $\log g, 0.08 \mathrm{dex}$ on $[\mathrm{Fe} / \mathrm{H}]$, and $0.5 \mathrm{~km} \mathrm{~s}^{-1}$ on $v \sin i$, which reflects the systematic uncertainty in the method, and is based on applying the SPC analysis to observations of spectroscopic standard stars. Following
Torres et al. (2012), we then revised the atmospheric parameters of the stars in an iterative fashion. We carried out a joint analysis of the light curves and RV curves to determine the mean stellar density $\rho_{\star}$ for each host. We then combined the 

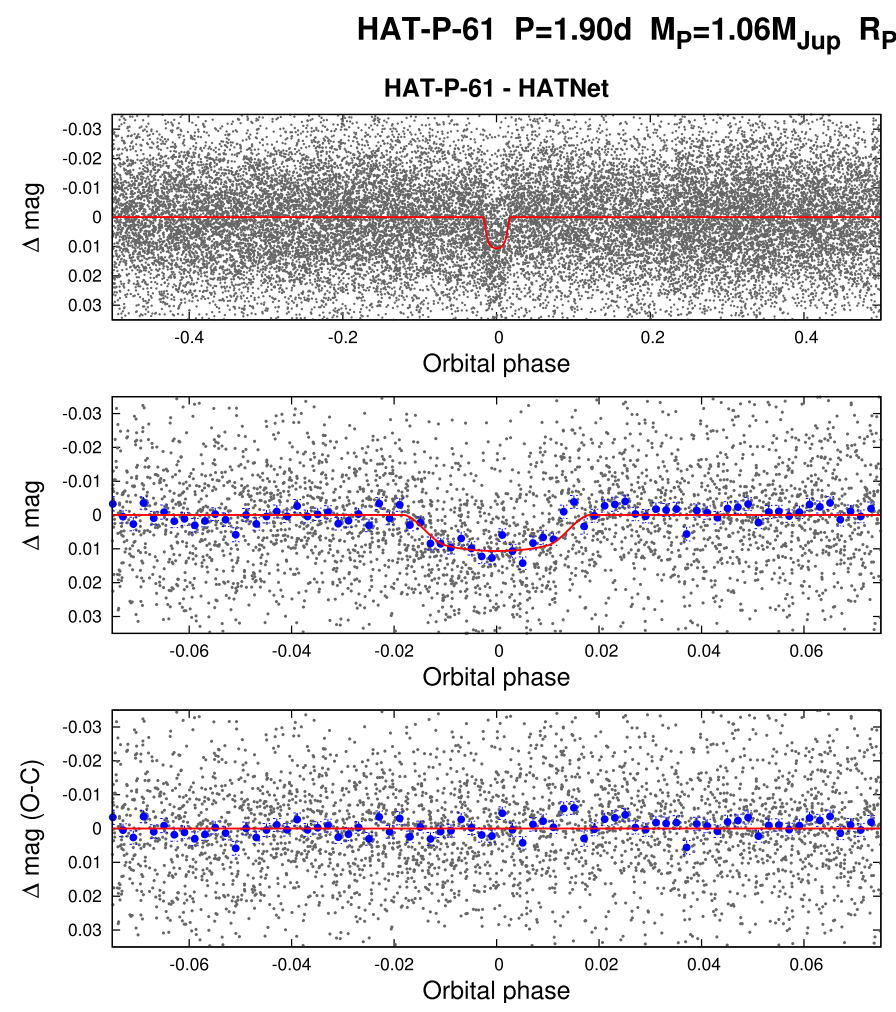

$=0.90 R_{\text {Jup }} M_{S}=1.00 M_{\text {Sun }} R_{S}=0.94 R_{\text {Sun }}$
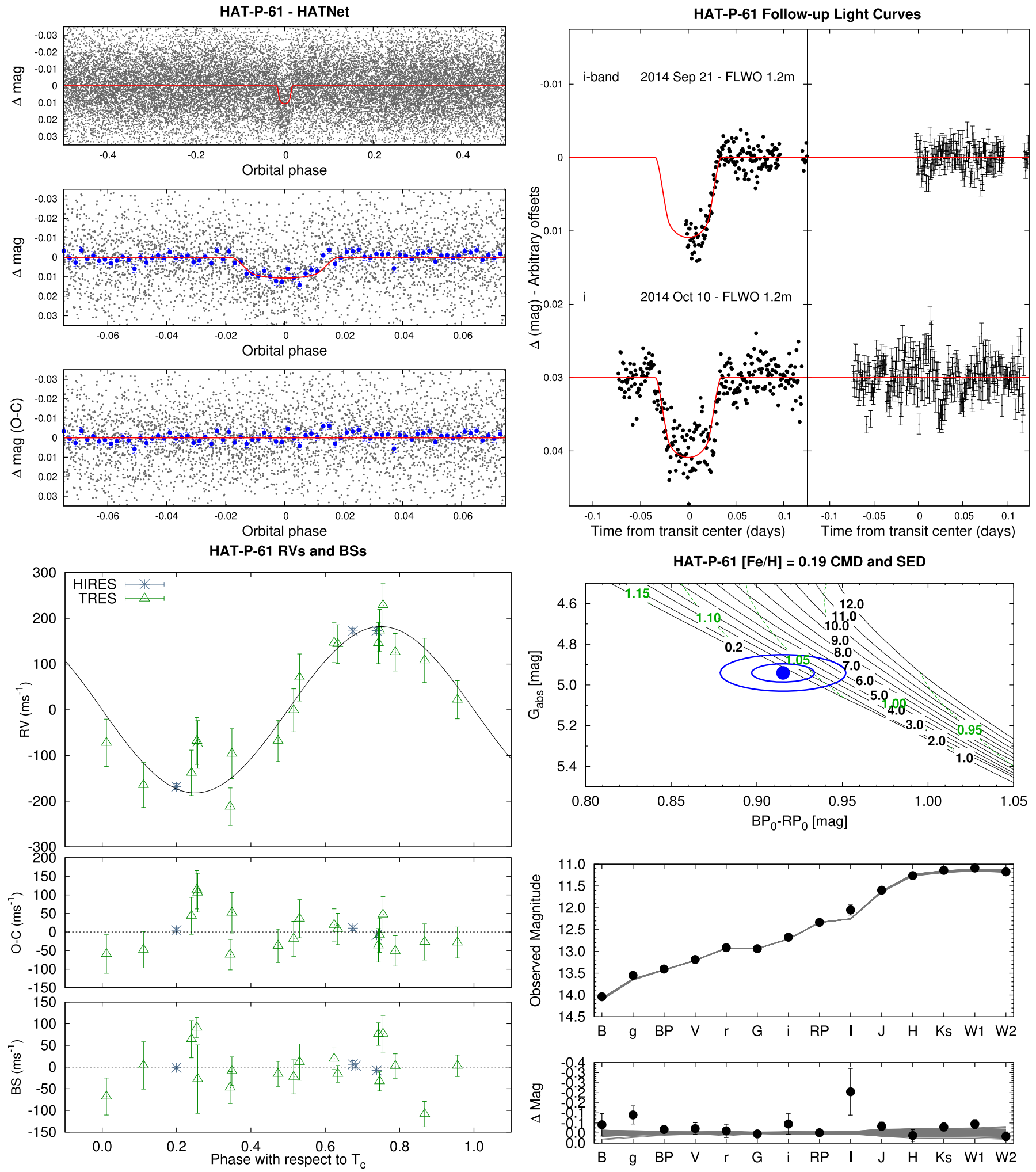

Figure 9. Observations of HAT-P-61 together with our best-fit model. See Figure 1 for a more detailed caption. The TESS light curve for this system is shown in Figure 15.

$T_{\text {eff } \star}$ and $[\mathrm{Fe} / \mathrm{H}]$ from the spectra with $\rho_{\star}$ to determine the surface gravities via interpolation within the Yonsei-Yale theoretical stellar isochrones (Yi et al. 2001). The surface gravities were then fixed to the values from this procedure in a second iteration of the SPC where only $T_{\mathrm{eff} \star},[\mathrm{Fe} / \mathrm{H}]$ and $v \sin i$ were allowed to vary. Note that this procedure for determining the fixed value of $\log g_{\star}$ was performed prior to the release of Gaia DR2, and we chose not to perform an additional iteration 


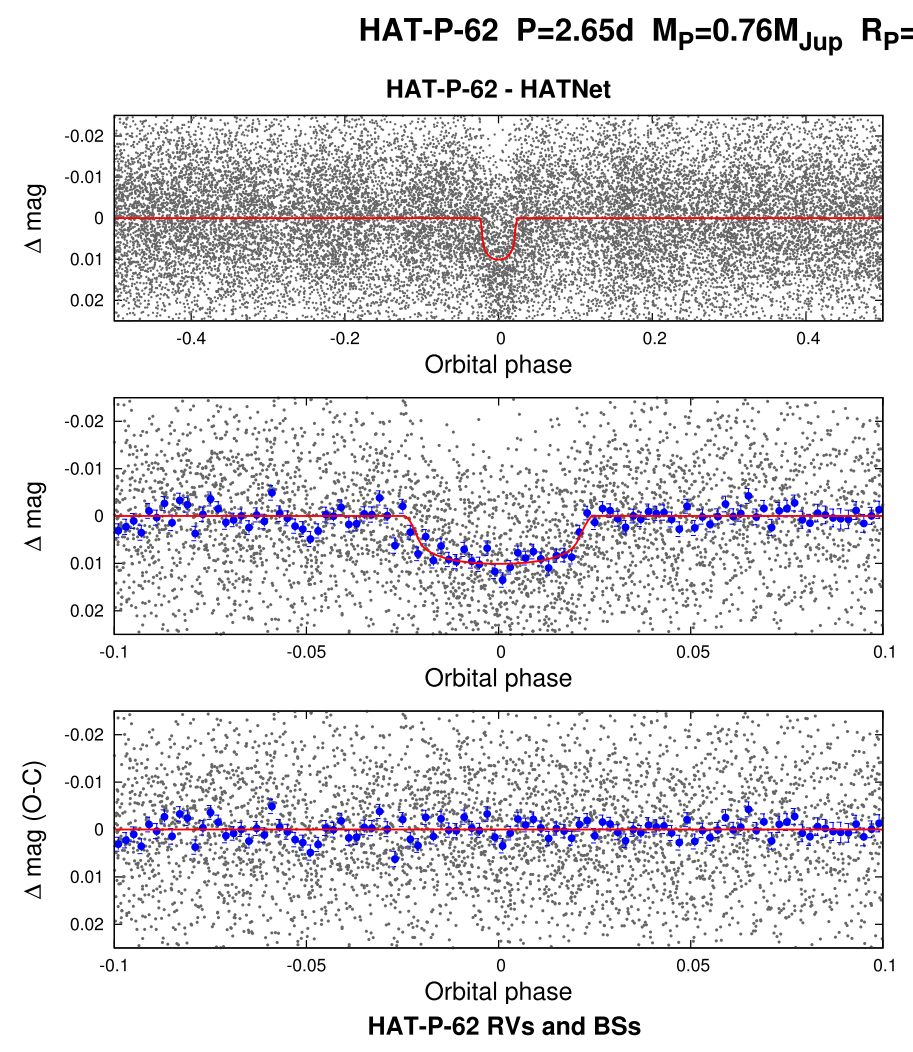

$$
\begin{array}{r}
R_{P}=1.07 R_{\text {Jup }} M_{S}=1.02 M_{\text {Sun }} R_{S}=1.17 R_{\text {Sun }} \\
\text { HAT-P-62 Follow-up Light Curves }
\end{array}
$$

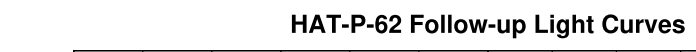

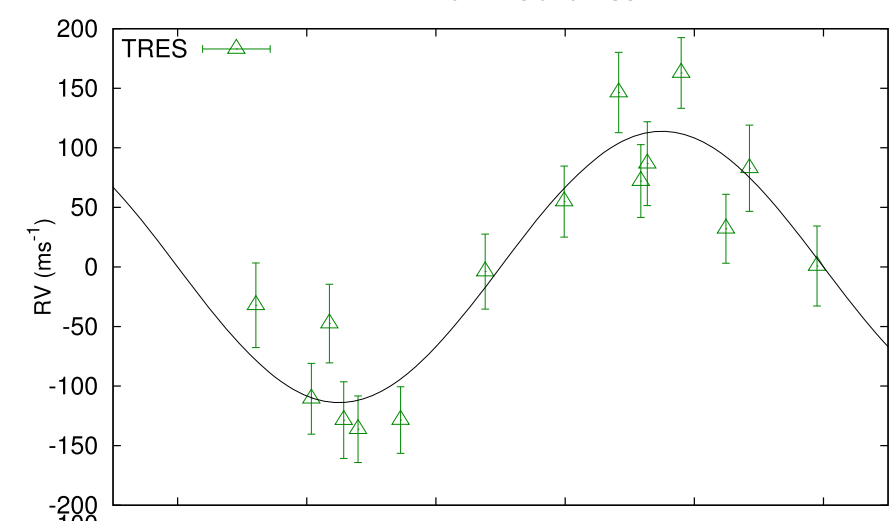
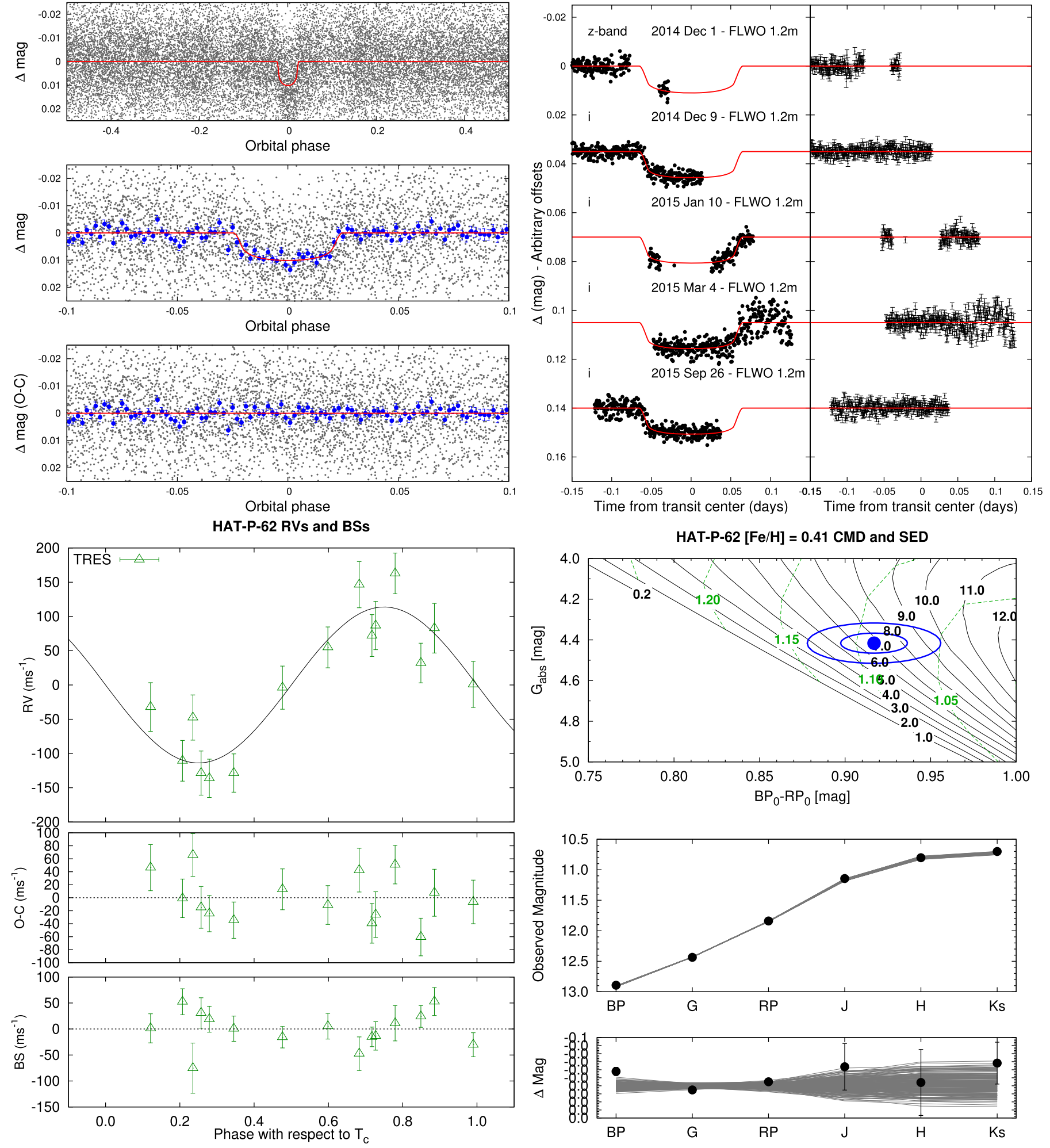

Figure 10. Observations of HAT-P-62 together with our best-fit model. See Figure 1 for a more detailed caption.

of the SPC making use of the Gaia DR2 parallax. The expected change in the atmospheric parameters were in all cases smaller than the systematic uncertainties.
The final spectroscopic parameters, together with catalog astrometry and photometry are listed for the host stars in Tables 6 and 7. 

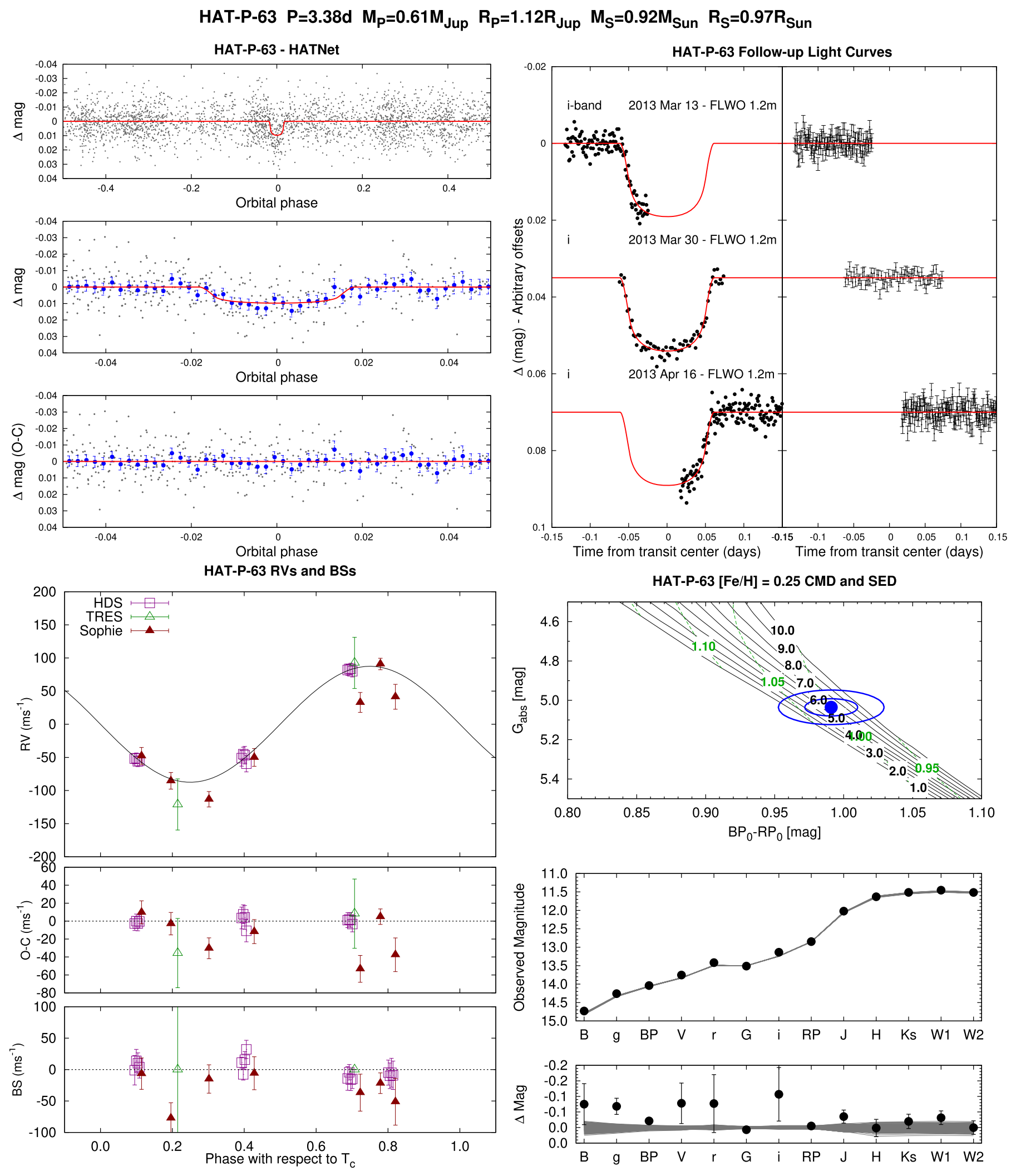

Figure 11. Observations of HAT-P-63 together with our best-fit model. See Figure 1 for a more detailed caption.

The final atmospheric parameters are then treated as observations which are simultaneously fitted, together with the light curves, RV curves, parallaxes, and catalog broadband photometry as described in Section 3.3. Here the fitting procedure makes use of the PARSEC stellar evolution models (Marigo et al. 2017) to constrain the physical properties of the stars. The final derived physical parameters of the stars, based on this method, including $M_{\star}, R_{\star}, \log g_{\star}, \rho_{\star}, L_{\star}, T_{\text {eff } \star},[\mathrm{Fe} / \mathrm{H}]$, the 

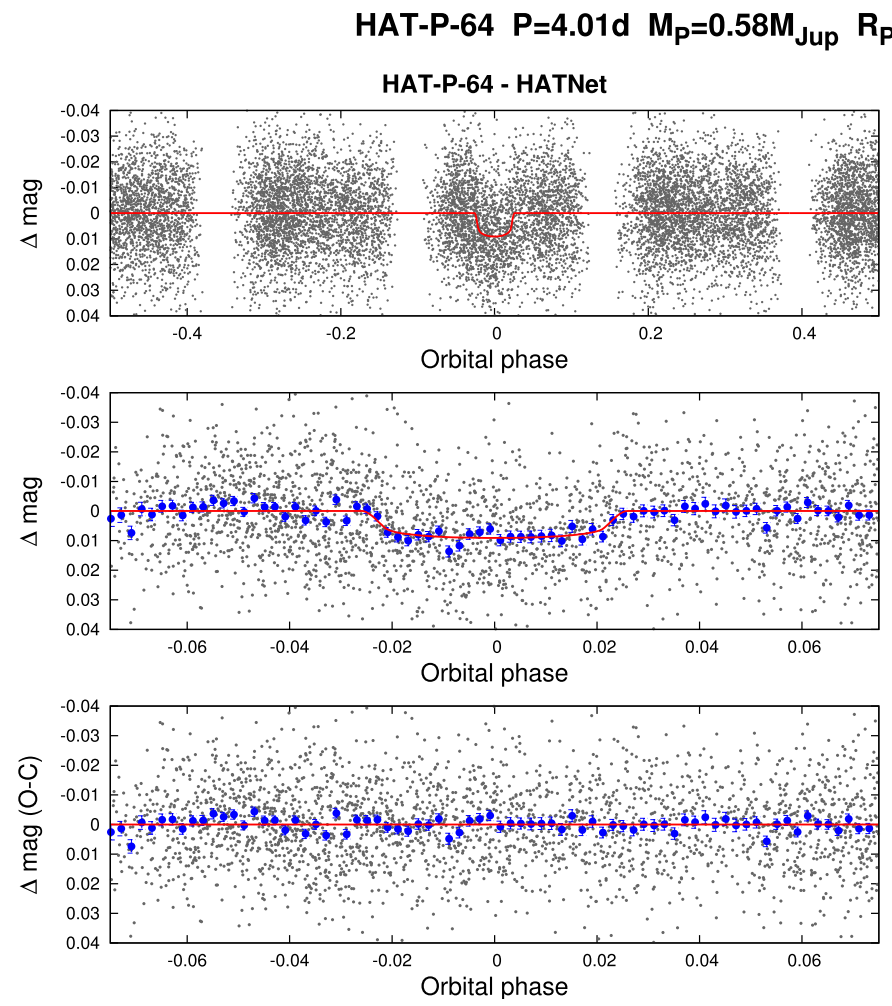

$R_{P}=1.70 R_{\text {Jup }} M_{S}=1.30 M_{\text {Sun }} R_{S}=1.73 R_{\text {Sun }}$
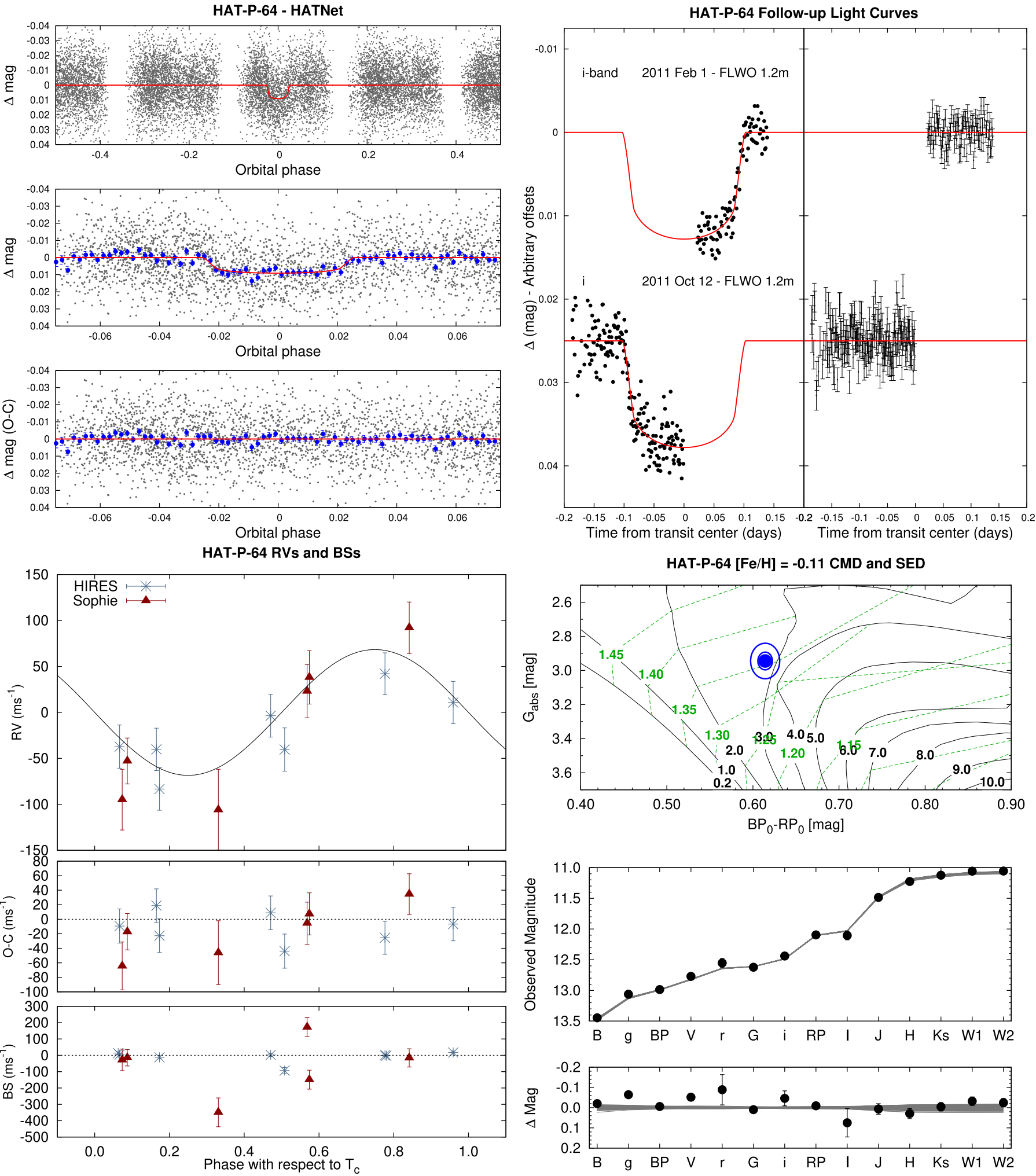

Figure 12. Observations of HAT-P-64 together with our best-fit model. See Figure 1 for a more detailed caption. The TESS light curve for this system is shown in Figure 16.

age of the system, the $V$-band extinction $\mathrm{A}_{V}$, and the distance to the system are listed in Tables 8 and 9 . Note that the values of $T_{\text {eff } \star}$ and $[\mathrm{Fe} / \mathrm{H}]$ listed here are the optimized values that are varied in the joint analysis, and may differ from the values for those parameters determined from modeling the spectra listed in Tables 6 and 7. Figures 1 and 7-12 show the de-reddened Gaia 

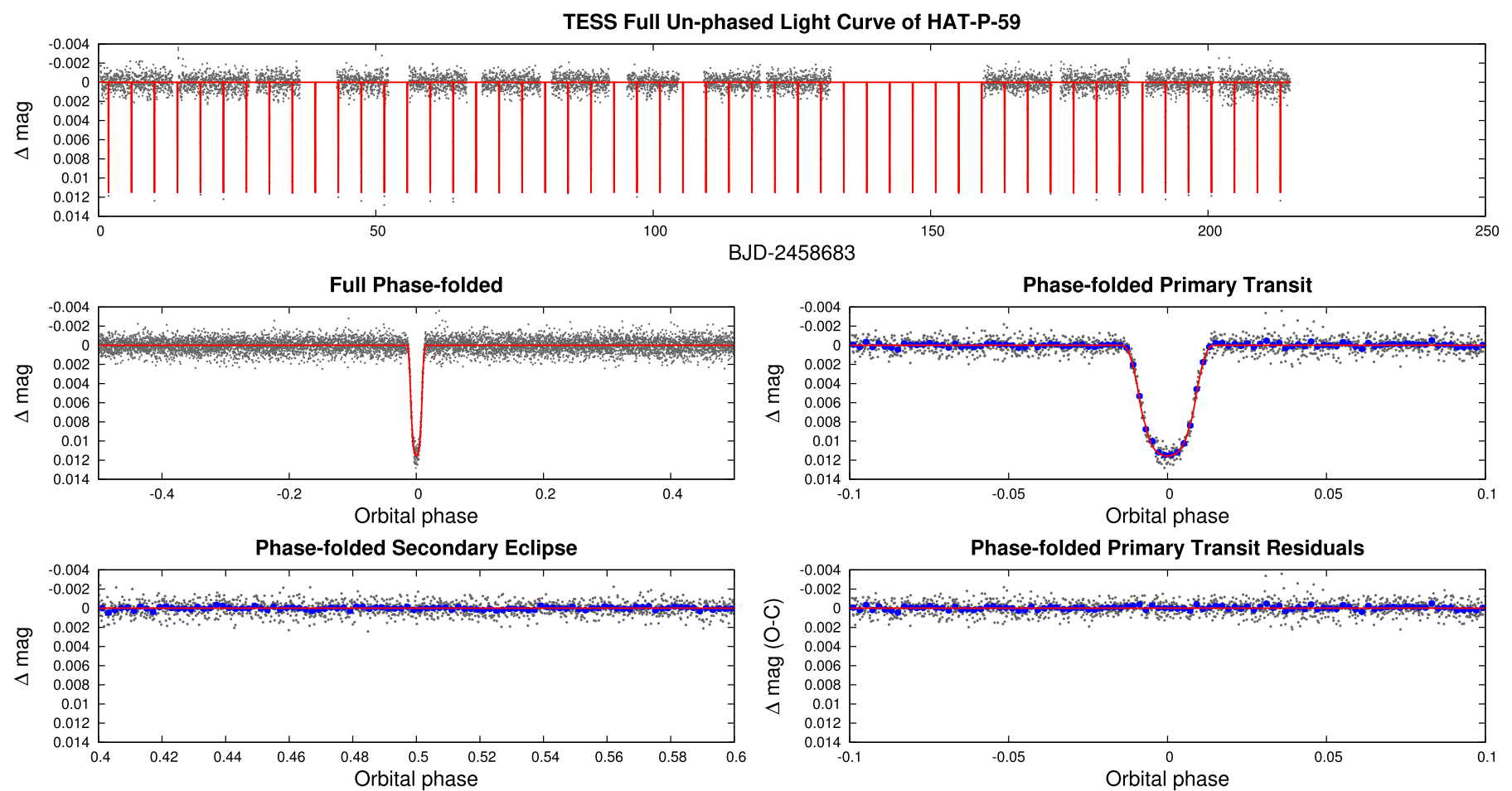

Figure 13. Similar to Figure 2, here we show the TESS long-cadence light curve for HAT-P-59. Other observations included in our analysis of this system are shown in Figure 7.

TESS Full Un-phased Light Curve of HAT-P-60

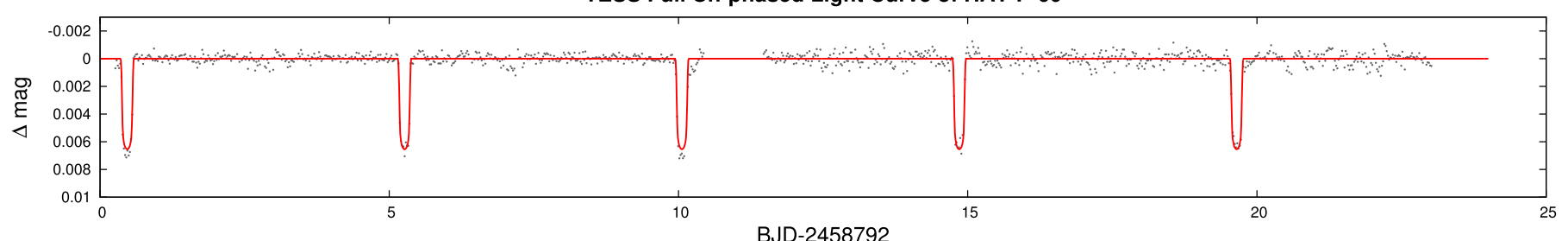

Full Phase-folded
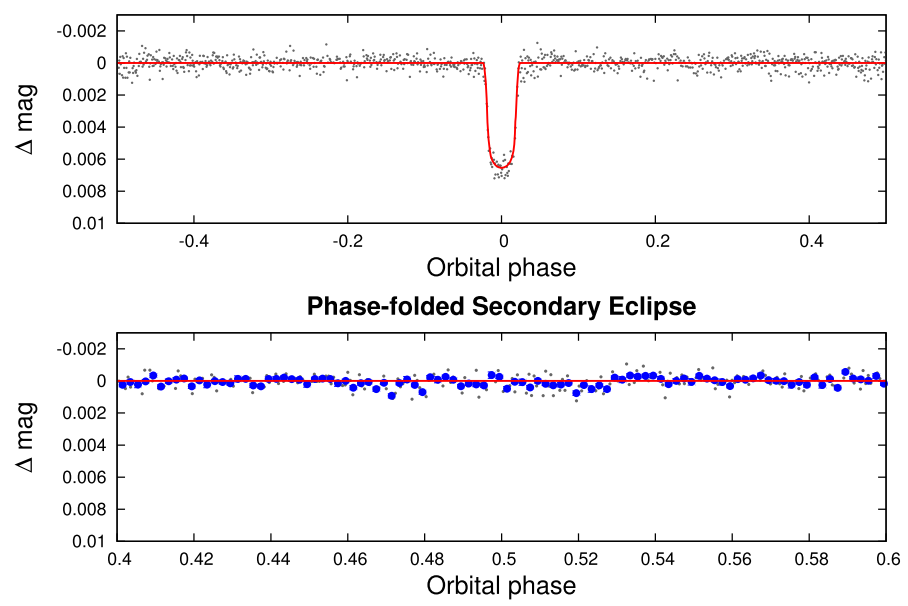
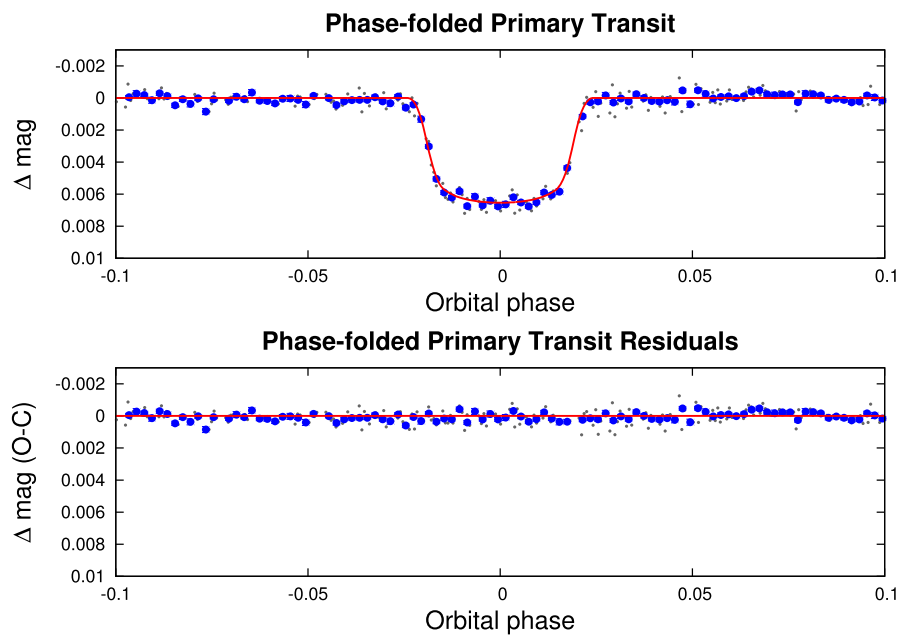

Figure 14. Similar to Figure 2, here we show the TESS long-cadence light curve for HAT-P-60. Other observations included in our analysis of this system are shown in Figure 8.

DR2 $B P-R P$ colors versus absolute $G$ magnitudes for each star compared to the PARSEC stellar evolution models, and also show the broadband spectral energy distribution (plotted as magnitude versus filter) of each star compared to the PARSEC models. We find that the best-fit models are in reasonably good agreement with the observations for all host stars. For example, the resulting derived distance measurements are within $1 \sigma$ of the values determined solely from the Gaia DR2 parallax measurements for all seven systems. While individual photometric or spectroscopic measurements may differ by as much as $3 \sigma$ from the model for some systems (e.g., the derived $[\mathrm{Fe} / \mathrm{H}]$ metallicity of $-0.224 \pm 0.057$ versus spectroscopically observed $[\mathrm{Fe} / \mathrm{H}]$ 

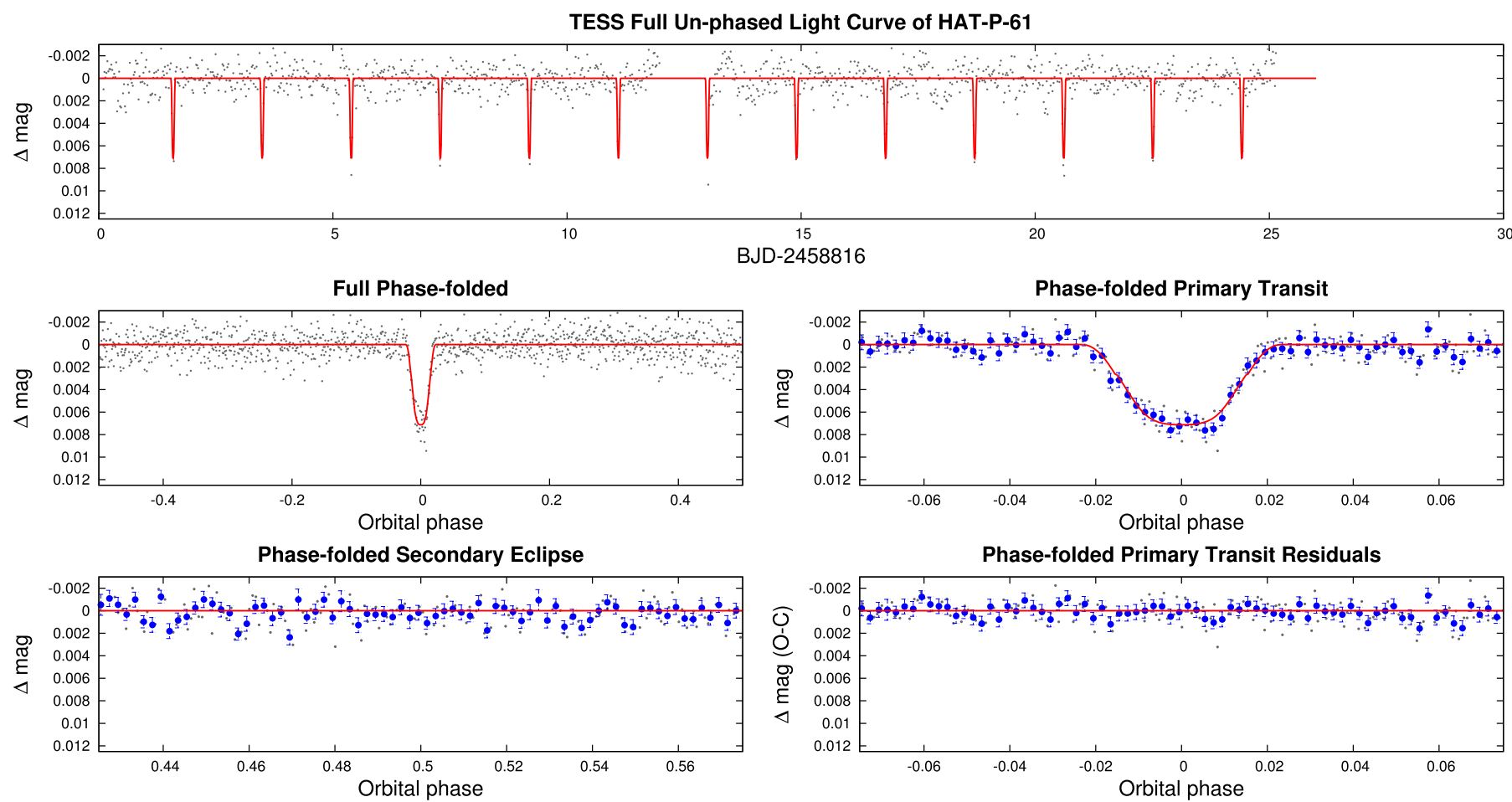

Figure 15. Similar to Figure 2, here we show the TESS long-cadence light curve for HAT-P-61. Other observations included in our analysis of this system are shown in Figure 9.
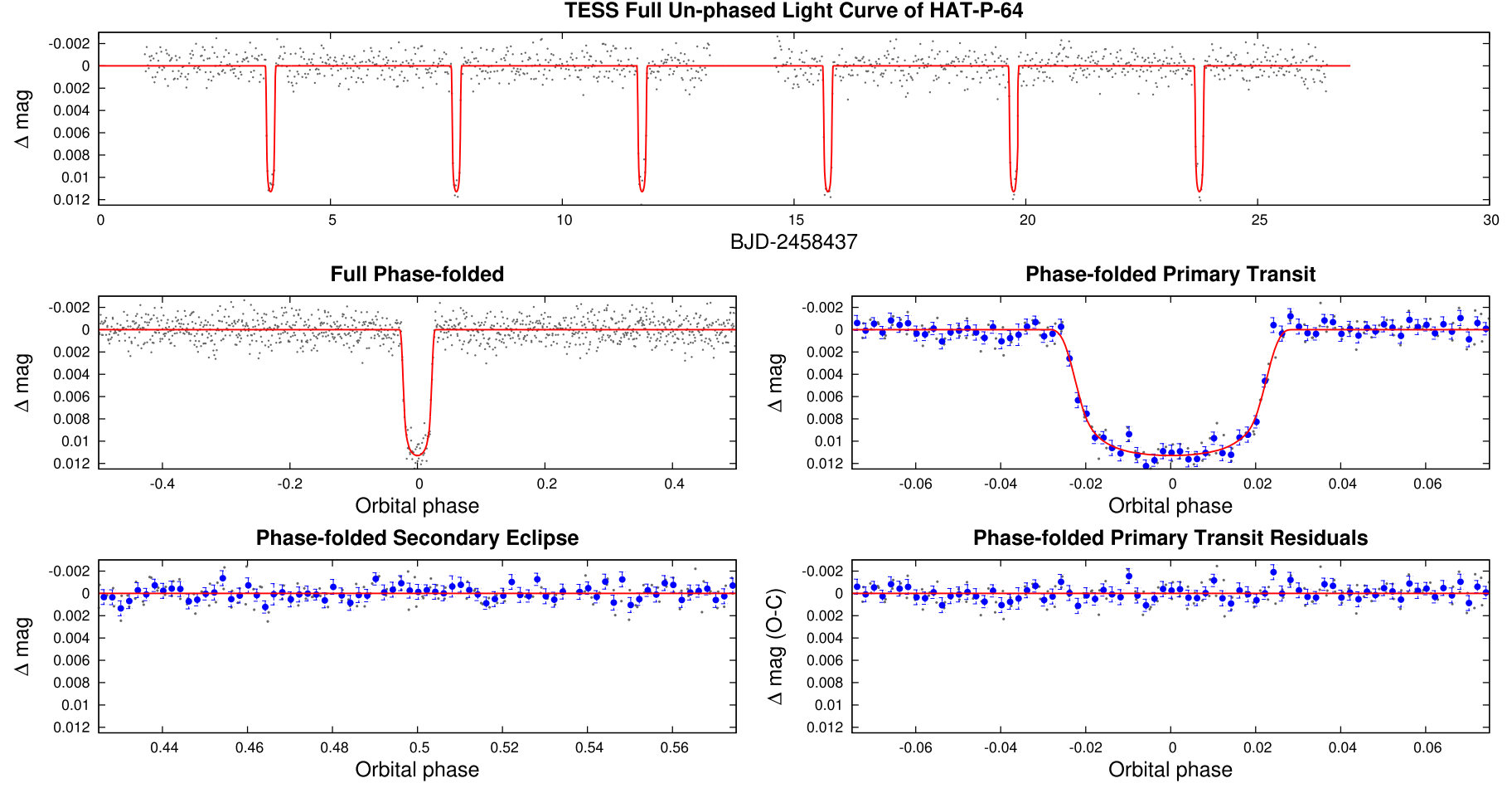

Figure 16. Similar to Figure 2, here we show the TESS long-cadence light curve for HAT-P-64. Other observations included in our analysis of this system are shown in Figure 12.

metallicity of $0.012 \pm 0.080$ for HAT-P-58), discrepancies at this level are common when jointly fitting all of the stellar data using isochrones to constrain the stellar properties. These differences are most likely due to underestimated systematic errors in (some of) the measurements, and/or systematic errors in the isochrone models. 

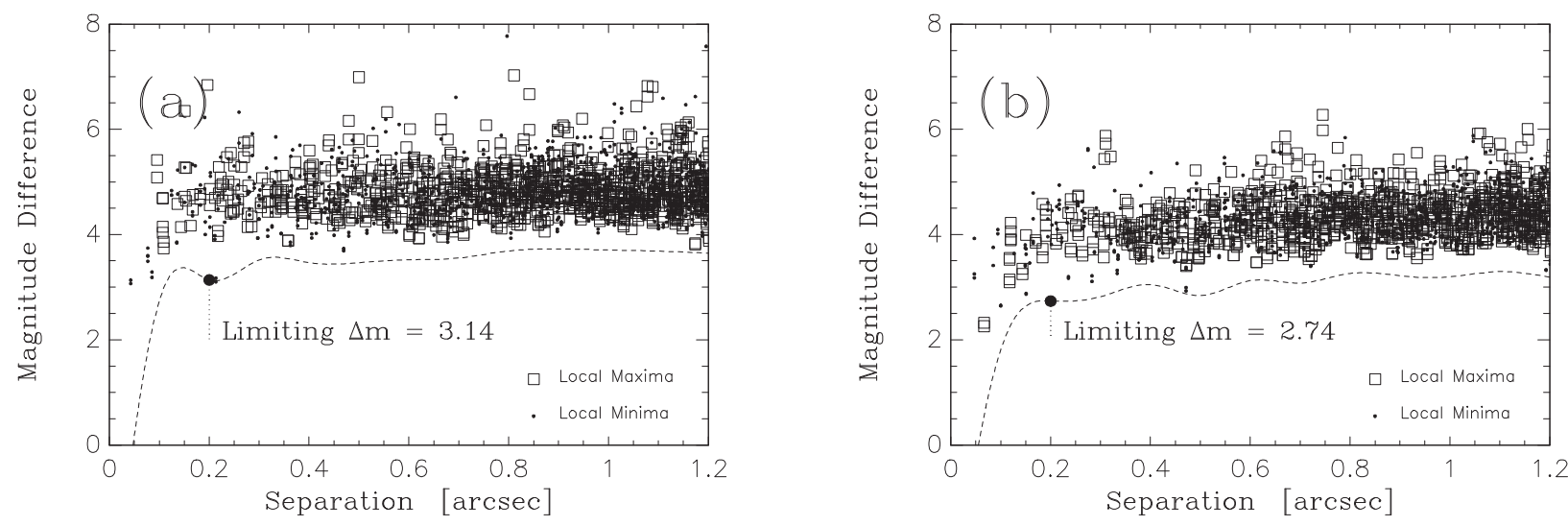

Figure 17. Same as Figure 4, here we show the results for HAT-P-59.
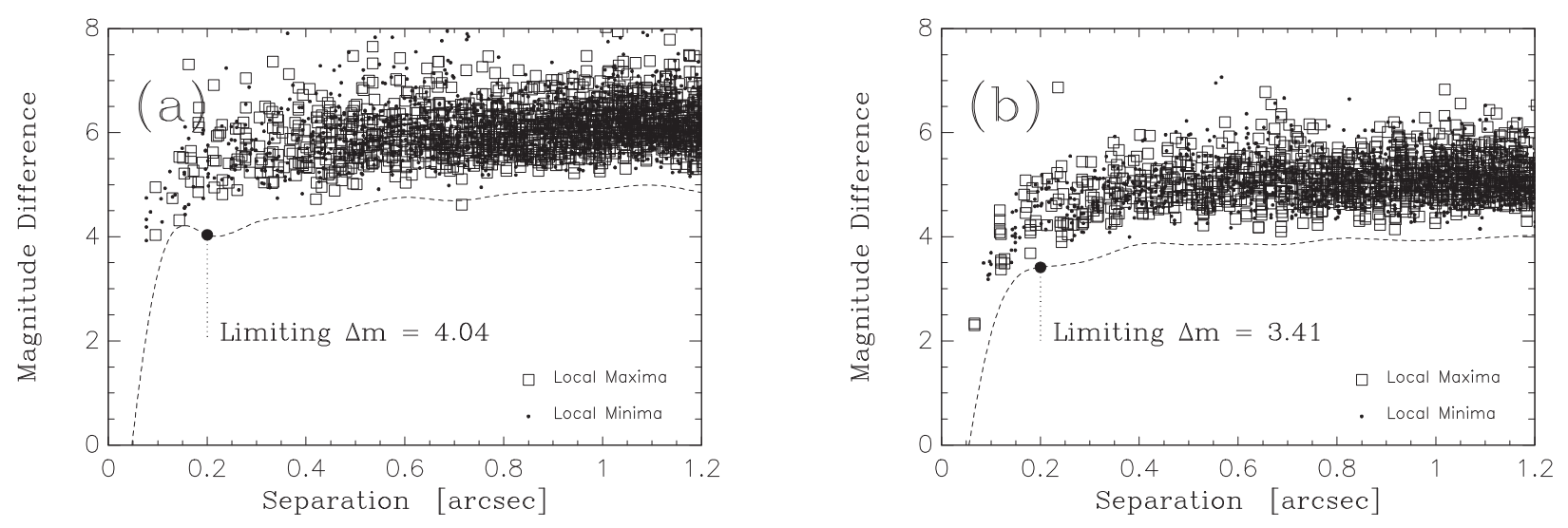

Figure 18. Same as Figure 4, here we show the results for HAT-P-60.

Table 5

Box Least-squares Search for Additional Transits in the Residual TESS Light Curves

\begin{tabular}{lccccc}
\hline \hline System & $\begin{array}{c}\text { Period } \\
(\text { days })\end{array}$ & $\begin{array}{c}T_{C} \\
\left(\mathrm{BJD}_{\mathrm{TDB}}-245000\right)\end{array}$ & $\begin{array}{c}\text { Duration } \\
(\mathrm{hr})\end{array}$ & $\begin{array}{c}\text { Depth } \\
(\mathrm{mmag})\end{array}$ & $N_{\text {transits }}$ \\
\hline HAT-P-58 & $22.130^{\mathrm{b}}$ & 8829.949 & 20.5 & 1.4 & 1 \\
HAT-P-59 & 19.956 & 8702.753 & 10.1 & 0.48 & 8 \\
HAT-P-60 & 6.7248 & 8799.080 & 6.9 & 0.45 & 7.75 \\
HAT-P-61 & 17.447 & 8816.517 & 8.3 & 1.4 & 2 \\
HAT-P-64 & 0.2151 & 8438.105 & 0.072 & 1.4 & 17 \\
\hline
\end{tabular}

Notes.

a The signal-to-pink-noise ratio as calculated by VARTOOLS (Hartman \& Bakos 2016).

${ }^{\mathrm{b}}$ In this case only a single transit event is identified by BLS, and the period is not meaningful.

\subsection{Excluding Blend Scenarios}

In order to exclude blend scenarios we carried out an analysis following Hartman et al. (2012), as updated in Hartman et al. (2019). Here we attempt to model the available photometric data (including light curves and catalog broadband photometric measurements) for each object as a blend between an eclipsing binary star system and a third star along the line of sight (either a physical association or a chance alignment). The physical properties of the stars are constrained using the Padova isochrones (Girardi et al. 2002), while we also require that the brightest of the three stars in the blend have atmospheric parameters consistent with those measured with the SPC. We also simulate composite cross-correlation functions and use them to predict RVs and BSs for each blend scenario considered.

Based on this analysis we rule out blended stellar eclipsing binary scenarios for all seven systems. The results for each object are as follows.

1. HAT-P-58: All blend models tested yield higher $\chi^{2}$ fits to the photometry than the model of a single star with a transiting planet, and can be rejected with $\sim 1 \sigma$ confidence. Those models that cannot be rejected with at least $5 \sigma$ confidence based solely on the photometry predict BS variations in excess of $1 \mathrm{~km} \mathrm{~s}^{-1}$ (however, the measured BS rms scatter from HIRES is $21 \mathrm{~m} \mathrm{~s}^{-1}$ ). 

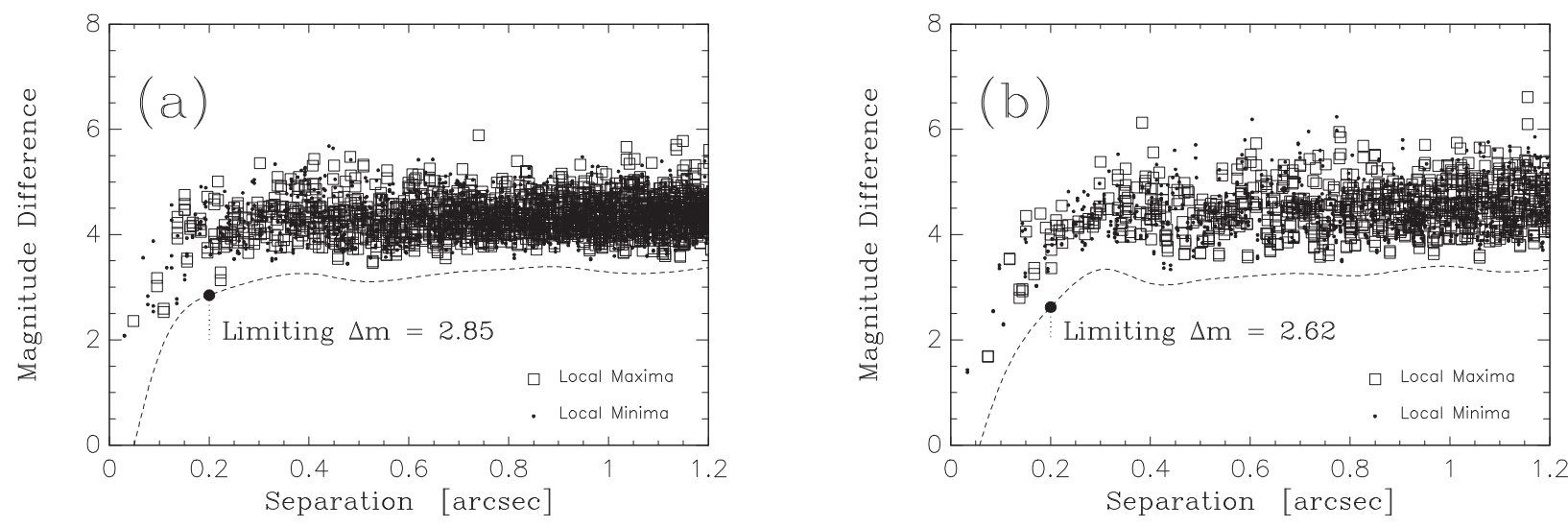

Figure 19. Same as Figure 4, here we show the results for HAT-P-61.
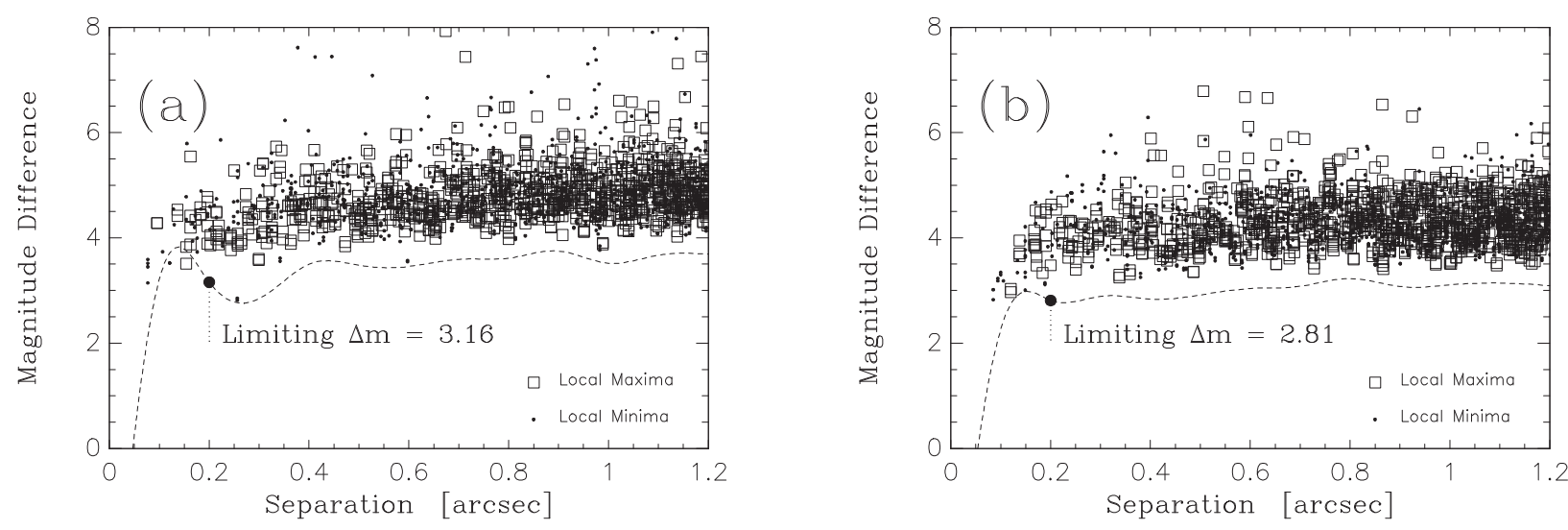

Figure 20. Same as Figure 4, here we show the results for HAT-P-62.
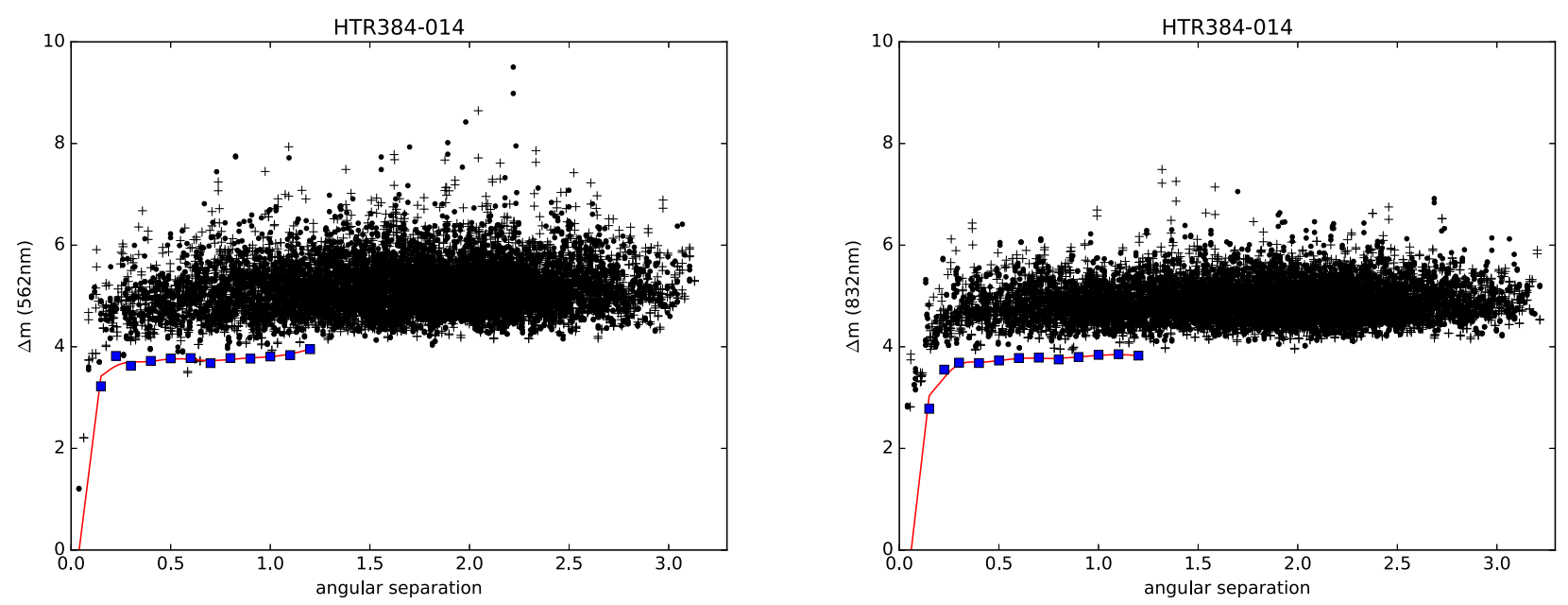

Figure 21. Similar to Figure 4, here we show the results for HAT-P-63 obtained with the NESSI instrument on the WIYN 3.5 m. For this instrument the filters used have wavelengths of $562 \mathrm{~nm}$ (left) and $832 \mathrm{~nm}$ (right).

2. HAT-P-59: All blend models tested yield higher $\chi^{2}$ fits to the photometry than the model of a single star with a transiting planet, and can be rejected with $3 \sigma$ confidence. Those models that cannot be rejected with at least $5 \sigma$ confidence based solely on the photometry predict BS variations in excess of $100 \mathrm{~m} \mathrm{~s}^{-1}$ (however, the measured BS rms scatter from TRES is $50 \mathrm{~m} \mathrm{~s}^{-1}$ ) and RV variations that do not reproduce the observed sinusoidal variation.
3. HAT-P-60: All blend models tested can be rejected with at least $5 \sigma$ confidence based solely on the photometry.

4. HAT-P-61: Similar to HAT-P-59, all blend models tested yield higher $\chi^{2}$ fits to the photometry than the model of a single star with a transiting planet, and can be rejected with $2 \sigma$ confidence based on the photometry alone. Those models that cannot be rejected with at least $5 \sigma$ confidence based solely on the photometry predict HIRES BS 

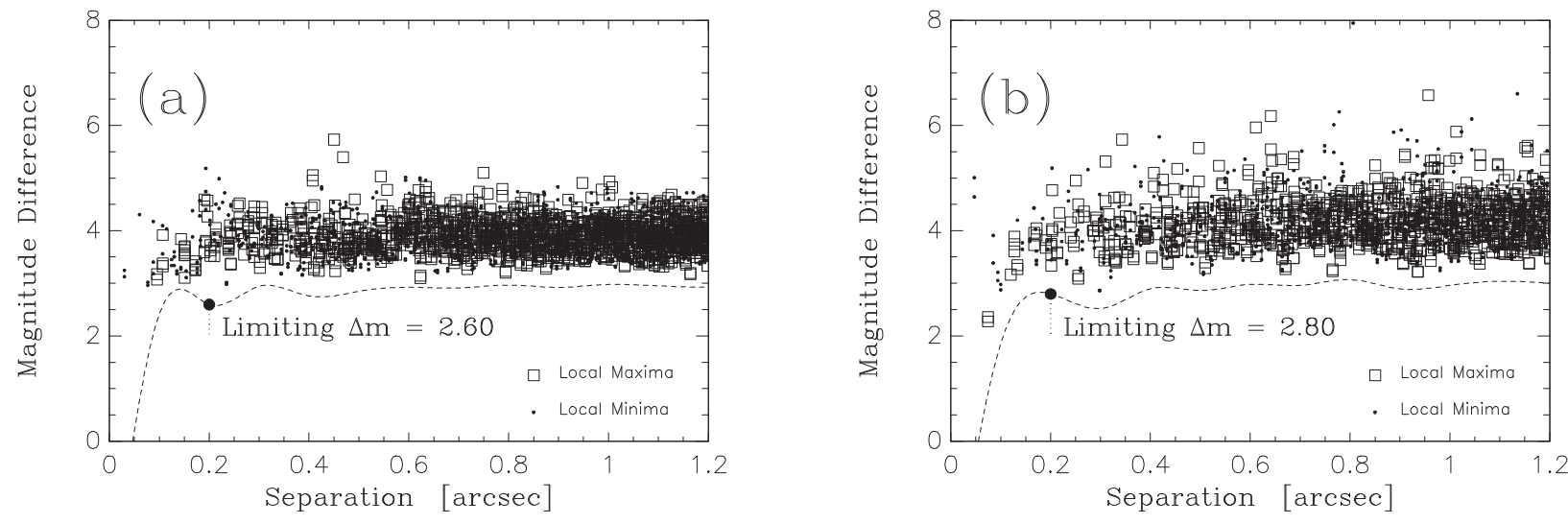

Figure 22. Same as Figure 4, here we show the results for HAT-P-64.

variations in excess of $100 \mathrm{~m} \mathrm{~s}^{-1}$ (the measured $\mathrm{BS} \mathrm{rms}$ scatter from HIRES is $5 \mathrm{~m} \mathrm{~s}^{-1}$ ), TRES BS variations in excess of $200 \mathrm{~m} \mathrm{~s}^{-1}$ (the measured BS rms scatter from TRES is $50 \mathrm{~m} \mathrm{~s}^{-1}$ ), and RV variations that do not reproduce the observed sinusoidal variation.

5. HAT-P-62: All blend models tested have higher $\chi^{2}$ fits to the photometry than the model of a single star with a transiting planet, and can be rejected with at least $1 \sigma$ confidence. Those models that cannot be rejected with at least $5 \sigma$ confidence can be rejected based on the BS observations. These blend models yield an rms scatter for the BSs in excess of $390 \mathrm{~m} \mathrm{~s}^{-1}$, whereas the measured TRES BS rms scatter is $35 \mathrm{~m} \mathrm{~s}^{-1}$.

6. HAT-P-63: Similar to HAT-P-59, all blend models tested yield higher $\chi^{2}$ fits to the photometry than the model of a single star with a transiting planet, and can be rejected with $1.5 \sigma$ confidence based on the photometry alone. Those models that cannot be rejected with at least $5 \sigma$ confidence based solely on the photometry predict HDS BS variations in excess of $60 \mathrm{~m} \mathrm{~s}^{-1}$ (the measured BS rms scatter from HDS is $\left.13 \mathrm{~m} \mathrm{~s}^{-1}\right)$, SOPHIE BS variations in excess of $400 \mathrm{~m} \mathrm{~s}^{-1}$ (the measured BS rms scatter from SOPHIE is $26 \mathrm{~m} \mathrm{~s}^{-1}$ ), and $\mathrm{RV}$ variations in excess of $\sim 200 \mathrm{~m} \mathrm{~s}^{-1}$ that do not reproduce the observed sinusoidal variation.

7. HAT-P-64: All blend models tested have higher $\chi^{2}$ fits to the photometry than the model of a single star with a transiting planet, and can be rejected with at least $1 \sigma$ confidence. Those models that cannot be rejected with at least $5 \sigma$ confidence predict a $\mathrm{BS}$ rms scatter of at least $160 \mathrm{~m} \mathrm{~s}^{-1}$, compared to the measured BS rms of $35 \mathrm{~m} \mathrm{~s}^{-1}$ for the Keck/HIRES observations.

The analysis described above was carried out before the release of Gaia DR2 or TESS data. The consistency between the distance inferred for each source by this method, assuming it is a single star with a planet, and the Gaia DR2 distance only bolsters the basic conclusion that none of these systems is a blended stellar eclipsing binary. Moreover, the TESS light curves showed no features (such as secondary eclipses or large ellipsoidal variations) that would be indicative of a blended eclipsing binary that might motivate a re-analysis.

\subsection{Global Modeling of the Data}

In order to determine the physical parameters of the TEP systems, we carried out a global modeling of the HATNet,
KeplerCam, and TESS photometry, the high-precision RV measurements, the SPC $T_{\text {eff }}$ and $[\mathrm{Fe} / \mathrm{H}]$ measurements, the Gaia DR2 parallax, and the Gaia DR2, APASS, TASS Mark IV, 2MASS, and WISE broadband photometry $(G, B P, R P, B$, $V, g, r, i, R, I_{C}, J, H, K_{S}, W_{1}, W_{2}, W_{3}, W_{4}$; where available).

We fit Mandel \& Agol (2002) transit models to the light curves assuming quadratic limb darkening. The limb-darkening coefficients are allowed to vary in the fit, but we use the tabulations from Claret et al. $(2012,2013)$ and Claret (2018) to place informative Gaussian prior constraints on their values, assuming a prior uncertainty of 0.2 for each coefficient.

We allow for a dilution of the HATNet transit depth in cases where there are neighbors blended with the targets in the low spatial resolution survey images (HAT-P-61-HAT-P-64). For TESS we allow for dilution for all five observed systems, and also binned the model to account for the 30 minute exposure time (Kipping 2010). For the KeplerCam light curves we include a quadratic trend in time, linear trends with up to three parameters describing the shape of the point-spread function (PSF), and a simultaneous application of the TFA (Kovács et al. 2005) in our model for each event to correct for systematic errors in the photometry. For HAT-P-62 we also include dilution factors in the KeplerCam model to account for the blending with the 5!"21 neighbor. To do this we simulate KeplerCam images of the primary target and its neighbor using the observed PSF and drawing $i$-band magnitudes for each component from normal distributions with means and standard deviations based on the measured $i$ magnitudes for each source from APASS. We also simulate images without the neighbor. We then carry out aperture photometry on the simulated images and compare the flux measured with and without the neighbor to determine the expected dilution. The median and standard deviation of the dilution are then calculated from all simulations for a given night to establish Gaussian priors which are placed on the dilution parameters which we vary in our modeling.

We fit Keplerian orbits to the RV curves allowing the zeropoint for each instrument to vary independently in the fit, and allowing for RV jitter which we also vary as a free parameter for each instrument.

To model the additional stellar atmospheric, parallax, and photometry observations we introduce four new model parameters which are allowed to vary in the fit: the distance modulus $(m-M)_{0}$, the $V$-band extinction $A_{V}$, and the stellar atmospheric parameters $T_{\text {eff } \star}$ and $[\mathrm{Fe} / \mathrm{H}]$. Each link in the 
Table 6

Astrometric, Spectroscopic, and Photometric Parameters for HAT-P-58, HAT-P-59, HAT-P-60, and HAT-P-61

\begin{tabular}{|c|c|c|c|c|c|}
\hline Parameter & $\begin{array}{c}\text { HAT-P-58 } \\
\text { Value }\end{array}$ & $\begin{array}{c}\text { HAT-P-59 } \\
\text { Value }\end{array}$ & $\begin{array}{l}\text { HAT-P-60 } \\
\text { Value }\end{array}$ & $\begin{array}{c}\text { HAT-P-61 } \\
\text { Value }\end{array}$ & Source \\
\hline \multicolumn{6}{|c|}{ Astrometric properties and cross-identifications } \\
\hline TIC-ID & 9443323 & 229400092 & 354469661 & 259506033 & \\
\hline TOI-ID & $\ldots$ & 1826.01 & 1580.01 & $\ldots$ & \\
\hline 2MASS-ID & $04352318+5652055$ & $19295008+6231452$ & $01530777+5203140$ & $05015525+5007526$ & \\
\hline GSC-ID & GSC 3740-01482 & GSC 4234-02195 & GSC 3292-01330 & GSC 3352-00595 & \\
\hline Gaia DR2-ID & 277493615044741376 & 2241743203599727744 & 359678187913760384 & 256580182331399296 & \\
\hline R.A. (ICRS J2015.5) & $04^{\mathrm{h}} 35^{\mathrm{m}} 23.1828 \mathrm{~s}$ & $19^{\mathrm{h}} 29^{\mathrm{m}} 50.0701 \mathrm{~s}$ & $01^{\mathrm{h}} 53^{\mathrm{m}} 07.7727 \mathrm{~s}$ & $05^{\mathrm{h}} 01^{\mathrm{m}} 55.2577 \mathrm{~s}$ & Gaia DR2 \\
\hline Decl. (ICRS J2015.5) & $+56^{\circ} 52^{\prime} 05^{\prime \prime} 5848$ & $+62^{\circ} 31^{\prime} 45^{\prime \prime} 1751$ & $+52^{\circ} 03^{\prime} 14^{\prime \prime} 01977$ & $+50^{\circ} 07^{\prime} 52^{\prime \prime} 5746$ & Gaia DR2 \\
\hline$\mu_{\text {R.A. }}\left({\left.\operatorname{mas~} \mathrm{yr}^{-1}\right)}^{-1}\right.$ & $-10.883 \pm 0.072$ & $20.957 \pm 0.046$ & $26.51 \pm 0.11$ & $-11.021 \pm 0.067$ & Gaia DR2 \\
\hline$\mu_{\text {decl. }}\left(\right.$ mas yr $\left.^{-1}\right)$ & $11.862 \pm 0.064$ & $-6.056 \pm 0.043$ & $6.165 \pm 0.075$ & $-21.440 \pm 0.063$ & Gaia DR2 \\
\hline Parallax (mas) & $1.912 \pm 0.047$ & $3.738 \pm 0.019$ & $4.260 \pm 0.049$ & $2.923 \pm 0.035$ & Gaia DR2 \\
\hline \multicolumn{6}{|l|}{ Spectroscopic properties } \\
\hline$T_{\text {eff } \star}(\mathrm{K})$ & $5931 \pm 50$ & $5665 \pm 50$ & $6462 \pm 50$ & $5551 \pm 50$ & $\mathrm{SPC}^{\mathrm{a}}$ \\
\hline$[\mathrm{Fe} / \mathrm{H}]$ & $0.012 \pm 0.080$ & $0.409 \pm 0.080$ & $-0.237 \pm 0.080$ & $0.396 \pm 0.080$ & SPC \\
\hline$v \sin i\left(\mathrm{~km} \mathrm{~s}^{-1}\right)$ & $4.91 \pm 0.50$ & $3.04 \pm 0.50$ & $10.42 \pm 0.50$ & $3.69 \pm 0.50$ & SPC \\
\hline$v_{\mathrm{mac}}\left(\mathrm{km} \mathrm{s}^{-1}\right)$ & 1.0 & 1.0 & 1.0 & 1.0 & Assumed \\
\hline$v_{\text {mic }}\left(\mathrm{km} \mathrm{s}^{-1}\right)$ & 2.0 & 2.0 & 2.0 & 2.0 & Assumed \\
\hline$\gamma_{\mathrm{RV}}\left(\mathrm{km} \mathrm{s}^{-1}\right)$ & $-35.97 \pm 0.10$ & $-20.477 \pm 0.027$ & $6.582 \pm 0.027$ & $4.810 \pm 0.022$ & TRES $^{\mathrm{b}}$ \\
\hline$S_{\mathrm{HK}}$ & $0.150 \pm 0.010$ & $\ldots$ & $0.1236 \pm 0.0022$ & $0.240 \pm 0.012$ & HIRES \\
\hline $\log R_{\mathrm{HK}}^{\prime}$ & $-5.057 \pm 0.072$ & $\cdots$ & $-5.309 \pm 0.033$ & $-4.719 \pm 0.032$ & HIRES \\
\hline \multicolumn{6}{|l|}{ Photometric properties } \\
\hline$G(\mathrm{mag})^{\mathrm{c}}$ & $12.72020 \pm 0.00020$ & $11.67870 \pm 0.00030$ & $9.56360 \pm 0.00030$ & $12.93860 \pm 0.00040$ & Gaia DR2 \\
\hline$B P(\mathrm{mag})^{\mathrm{c}}$ & $13.1422 \pm 0.0016$ & $12.0587 \pm 0.0011$ & $9.8631 \pm 0.0012$ & $13.4067 \pm 0.0018$ & Gaia DR2 \\
\hline$R P(\mathrm{mag})^{\mathrm{c}}$ & $12.13470 \pm 0.00090$ & $11.15850 \pm 0.00050$ & $9.1320 \pm 0.0013$ & $12.33560 \pm 0.00080$ & Gaia DR2 \\
\hline$B$ (mag) & $13.690 \pm 0.089$ & $12.581 \pm 0.094$ & $10.230 \pm 0.040$ & $14.040 \pm 0.056$ & APASS $^{\mathrm{d}}$ \\
\hline$V$ (mag) & $12.971 \pm 0.073$ & $11.883 \pm 0.065$ & $9.710 \pm 0.050$ & $13.188 \pm 0.029$ & APASS $^{\mathrm{d}}$ \\
\hline$I(\mathrm{mag})$ & $\ldots$ & $11.073 \pm 0.078$ & $9.077 \pm 0.042$ & $12.05 \pm 0.12$ & TASS Mark IV \\
\hline$g(\mathrm{mag})$ & $13.28 \pm 0.12$ & $12.16 \pm 0.10$ & $\ldots$ & $13.550 \pm 0.045$ & APASS $^{\mathrm{d}}$ \\
\hline$r(\mathrm{mag})$ & $12.74 \pm 0.12$ & $11.650 \pm 0.050$ & $\ldots$ & $12.915 \pm 0.033$ & APASS $^{\mathrm{d}}$ \\
\hline$i$ (mag) & $12.50 \pm 0.12$ & $11.478 \pm 0.050$ & $9.421 \pm 0.040$ & $12.675 \pm 0.051$ & APASS $^{\mathrm{a}}$ \\
\hline$J$ (mag) & $11.429 \pm 0.022$ & $10.581 \pm 0.020$ & $8.677 \pm 0.052$ & $11.598 \pm 0.021$ & 2 MASS $^{f}$ \\
\hline$H$ (mag) & $11.075 \pm 0.020$ & $10.268 \pm 0.018$ & $8.396 \pm 0.029$ & $11.263 \pm 0.029$ & 2 MASS $^{\mathrm{f}}$ \\
\hline$K_{s}(\mathrm{mag})$ & $10.978 \pm 0.023$ & $10.208 \pm 0.022$ & $8.368 \pm 0.031$ & $11.141 \pm 0.020$ & $2 \mathrm{MASS}^{\mathrm{f}}$ \\
\hline$W 1$ (mag) & $10.856 \pm 0.022$ & $10.144 \pm 0.023$ & $8.308 \pm 0.026$ & $11.089 \pm 0.022$ & WISE $^{\mathrm{f}}$ \\
\hline$W 2$ (mag) & $10.906 \pm 0.021$ & $10.211 \pm 0.020$ & $8.320 \pm 0.026$ & $11.176 \pm 0.022$ & WISE $^{\mathrm{f}}$ \\
\hline W3 (mag) & $10.359 \pm 0.061$ & $10.191 \pm 0.037$ & $8.260 \pm 0.028$ & $\ldots$ & WISE $^{\mathrm{f}}$ \\
\hline
\end{tabular}

Notes.

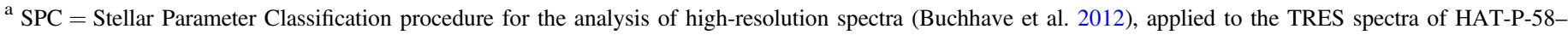

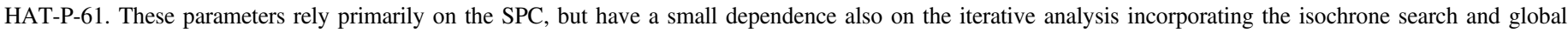
modeling of the data.

${ }^{\mathrm{b}}$ In addition to the uncertainty listed here, there is $\mathrm{a} \sim 0.1 \mathrm{~km} \mathrm{~s}^{-1}$ systematic uncertainty in transforming the velocities to the IAU standard system.

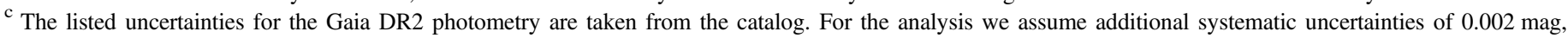
$0.005 \mathrm{mag}$, and $0.003 \mathrm{mag}$ for the $G-, B P$-, and $R P$-bands, respectively.

${ }^{\mathrm{d}}$ From APASS DR6 for as listed in the UCAC 4 catalog (Zacharias et al. 2013).

e From the Amateur Sky Survey (TASS) catalog release IV (Droege et al. 2006).

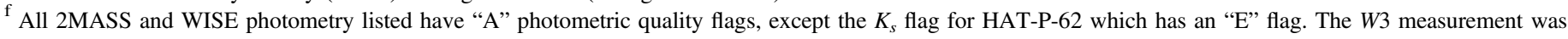
excluded from the analysis of systems for which this value is not listed. These were excluded due to high photometric uncertainties and/or poor-quality flags.

Markov chain yields a combination of $\left(T_{\mathrm{eff} \star}, \rho_{\star},[\mathrm{Fe} / \mathrm{H}]\right)$ which we use to determine the stellar mass, radius, $\log g$, luminosity, and absolute magnitude in various bandpasses by comparison with the PARSEC stellar evolution models (specifically PARSEC realease v1.2S + CLIBRI release PR16, as in Marigo et al. 2017) which we generated using the CMD 3.0 web interface by L. Girardi. ${ }^{24}$ Note that $\rho_{\star}$ is not varied directly in the fit, but rather can be computed from the other transit and orbital parameters which are varied. These absolute magnitudes, together with the model distance modulus and polynomial relations for the extinction in each bandpass as a

\footnotetext{
${ }^{24}$ http://stev.oapd.inaf.it/cgi-bin/cmd
}

function of $A_{V}$ and $T_{\text {eff } \star}$, are used to compute model values for the broadband photometry measurements to be compared to the observations. The polynomial relations for the extinction were determined based on tabulating the PARSEC isochrones at $A_{V}=1$ and at $A_{V}=0$, where the extinction coefficients are computed by the CMD interface on a star-by-star basis assuming the Cardelli et al. (1989) and O'Donnell (1994) $R_{V}=3.1$ extinction law. See Hartman et al. (2019) for details. Here we assume systematic errors of $0.002 \mathrm{mag}, 0.005 \mathrm{mag}$, and $0.003 \mathrm{mag}$ on the $G, B P$ and $R P$ photometry, respectively, following Evans et al. (2018). These systematic uncertainties are added in quadrature to the statistical uncertainties on the measurements listed in the Gaia DR2 catalog. 
Table 7

Astrometric, Spectroscopic, and Photometric Parameters for HAT-P-62, HAT-P-63, and HAT-P-64

\begin{tabular}{|c|c|c|c|c|}
\hline Parameter & $\begin{array}{c}\text { HAT-P-62 } \\
\text { Value }\end{array}$ & $\begin{array}{l}\text { HAT-P-63 } \\
\text { Value }\end{array}$ & $\begin{array}{c}\text { HAT-P-64 } \\
\text { Value }\end{array}$ & Source \\
\hline \multicolumn{5}{|c|}{ Astrometric properties and cross-identifications } \\
\hline TIC-ID & 453064665 & 1635721458 & 455036659 & \\
\hline 2MASS-ID & $04580102+4818038$ & $17581730+0545409$ & $04355384+0225526$ & \\
\hline GSC-ID & GSC 3348-01101 & GSC 0429-01697 & GSC 0086-00341 & \\
\hline Gaia DR2-ID & 255397142179844224 & 4474644332250439552 & 3279418602369232000 & \\
\hline$\mu_{\text {R.A. }}\left({\left.\operatorname{mas~} \mathrm{yr}^{-1}\right)}^{-1}\right.$ & $14.732 \pm 0.080$ & $-14.871 \pm 0.036$ & $7.784 \pm 0.079$ & Gaia DR2 \\
\hline$\mu_{\text {decl. }}\left(\operatorname{mas~yr}^{-1}\right)$ & $-43.776 \pm 0.061$ & $-0.301 \pm 0.039$ & $-3.863 \pm 0.044$ & Gaia DR2 \\
\hline parallax (mas) & $2.839 \pm 0.040$ & $2.450 \pm 0.024$ & $1.505 \pm 0.035$ & Gaia DR2 \\
\hline \multicolumn{5}{|l|}{ Spectroscopic properties } \\
\hline$T_{\text {eff } \star}(\mathrm{K})$ & $5601 \pm 50$ & $5365 \pm 50$ & $6302 \pm 50$ & $\mathrm{SPC}^{\mathrm{a}}$ \\
\hline$[\mathrm{Fe} / \mathrm{H}]$ & $0.449 \pm 0.080$ & $0.428 \pm 0.080$ & $-0.010 \pm 0.080$ & SPC \\
\hline$S_{\mathrm{HK}}$ & $\ldots$ & $\ldots$ & $0.1453 \pm 0.0068$ & HIRES \\
\hline $\log R_{\mathrm{HK}}^{\prime}$ & $\cdots$ & $\cdots$ & $-5.062 \pm 0.057$ & HIRES \\
\hline \multicolumn{5}{|l|}{ Photometric properties } \\
\hline$G(\mathrm{mag})^{\mathrm{c}}$ & $12.43620 \pm 0.00030$ & $13.51060 \pm 0.00030$ & $12.62210 \pm 0.00010$ & Gaia DR2 \\
\hline$B P(\mathrm{mag})^{\mathrm{c}}$ & $12.8932 \pm 0.0013$ & $14.0381 \pm 0.0012$ & $12.98580 \pm 0.00080$ & Gaia DR2 \\
\hline$R P(\mathrm{mag})^{\mathrm{c}}$ & $11.84000 \pm 0.00090$ & $12.84760 \pm 0.00080$ & $12.09530 \pm 0.00070$ & Gaia DR2 \\
\hline$B$ (mag) & $\ldots$ & $14.729 \pm 0.066$ & $13.446 \pm 0.011$ & APASS $^{\mathrm{d}}$ \\
\hline$V(\mathrm{mag})$ & $\ldots$ & $13.753 \pm 0.065$ & $12.771 \pm 0.010$ & $\operatorname{APASS}^{\mathrm{d}}$ \\
\hline$I$ (mag) & $\ldots$ & $\ldots$ & $12.105 \pm 0.070$ & TASS Mark IV \\
\hline$g$ (mag) & $\cdots$ & $14.258 \pm 0.026$ & $13.062 \pm 0.013$ & APASS $^{d}$ \\
\hline$r$ (mag) & $\ldots$ & $13.418 \pm 0.093$ & $12.553 \pm 0.075$ & APASS $^{\mathrm{d}}$ \\
\hline$i(\mathrm{mag})$ & $\ldots$ & $13.136 \pm 0.086$ & $12.440 \pm 0.037$ & APASS $^{\mathrm{d}}$ \\
\hline
\end{tabular}

Notes.

${ }^{a}$ SPC $=$ Stellar Parameter Classification procedure for the analysis of high-resolution spectra (Buchhave et al. 2012), applied to the TRES spectra of HAT-P-62 and HAT-P-63, and to the HIRES I ${ }_{2}$-free template spectra of HAT-P-64. These parameters rely primarily on the SPC, but have a small dependence also on the iterative analysis incorporating the isochrone search and global modeling of the data.

${ }^{\mathrm{b}}$ In addition to the uncertainty listed here, there is a $\sim 0.1 \mathrm{~km} \mathrm{~s}^{-1}$ systematic uncertainty in transforming the velocities to the IAU standard system.

${ }^{\mathrm{c}}$ The listed uncertainties for the Gaia DR2 photometry are taken from the catalog. For the analysis we assume additional systematic uncertainties of 0.002 mag, $0.005 \mathrm{mag}$, and $0.003 \mathrm{mag}$ for the $G-, B P$-, and $R P$-bands, respectively.

${ }^{\mathrm{d}}$ From APASS DR6 for as listed in the UCAC 4 catalog (Zacharias et al. 2013).

${ }^{\mathrm{e}}$ From the Amateur Sky Survey (TASS) catalog release IV (Droege et al. 2006).

f All 2MASS and WISE photometry listed have "A" photometric quality flags, except the $K_{s}$ flag for HAT-P-62 which has an "E" flag. The W3 measurement was excluded from the analysis of systems for which this value is not listed. These were excluded due to high photometric uncertainties and/or poor-quality flags.

For $A_{V}$ we make use of the MWDUST 3D Galactic extinction model (Bovy et al. 2016) to tabulate the extinction in $0.1 \mathrm{kpc}$ steps in the direction of the source. For a given $(m-M)_{0}$ we then perform linear interpolation among these values to estimate the expected $A_{V}$ at that distance. We treat this expected value as a Gaussian prior, with a $1 \sigma$ uncertainty of $20 \%$ the maximum value.

We use a differential evolution Markov chain Monte Carlo procedure to explore the fitness landscape and to determine the posterior distribution of the parameters. When a proposed link in the Markov chain falls outside of the parameter values spanned by the stellar evolution models (e.g., if a star with a density greater than what is allowed by the stellar evolution models at a given temperature and metallicity is proposed) the link is rejected and the previous link is retained. In this manner the fitting procedure used here forces the solutions to match to the theoretical stellar evolution models. We tried fitting both fixed-circular-orbit and free-eccentricity models to the data, and for all seven systems found that the data are consistent with a circular orbit. We therefore adopt the parameters that come from the fixed-circular-orbit models for all of the systems. The resulting parameters for HAT-P-58b, HAT-P-59b, HAT-P-60b, and HAT-P-61b are listed in Table 10, while for HAT-P-62b, HAT-P-63b, and HAT-P-64b they are listed in Table 11. 
Table 8

Derived Stellar Parameters for HAT-P-58, HAT-P-59, HAT-P-60, and HAT-P-61

\begin{tabular}{|c|c|c|c|c|}
\hline Parameter & $\begin{array}{c}\text { HAT-P-58 } \\
\text { Value }\end{array}$ & $\begin{array}{c}\text { HAT-P-59 } \\
\text { Value }\end{array}$ & $\begin{array}{c}\text { HAT-P-60 } \\
\text { Value }\end{array}$ & $\begin{array}{c}\text { HAT-P-61 } \\
\text { Value }\end{array}$ \\
\hline$M_{\star}\left(M_{\odot}\right)$ & $1.031 \pm 0.028$ & $1.008 \pm 0.022$ & $1.435 \pm 0.012$ & $1.004 \pm 0.033$ \\
\hline$R_{\star}\left(R_{\odot}\right)$ & $1.530 \pm 0.034$ & $1.1038 \pm 0.0073$ & $2.197_{-0.020}^{+0.027}$ & $0.938 \pm 0.011$ \\
\hline $\log g_{\star}(\mathrm{cgs})$ & $4.082 \pm 0.020$ & $4.356 \pm 0.013$ & $3.9114 \pm 0.0097$ & $4.496 \pm 0.021$ \\
\hline$\rho_{\star}\left(\mathrm{g} \mathrm{cm}^{-3}\right)$ & $0.405 \pm 0.026$ & $1.059 \pm 0.038$ & $0.1909_{-0.0071}^{+0.0054}$ & $1.715 \pm 0.094$ \\
\hline$L_{\star}\left(L_{\odot}\right)$ & $2.86 \pm 0.15$ & $1.132 \pm 0.015$ & $6.44 \pm 0.17$ & $0.767 \pm 0.031$ \\
\hline$T_{\text {eff } \star}(\mathrm{K})$ & $6078 \pm 48$ & $5678 \pm 16$ & $6212 \pm 26$ & $5587 \pm 45$ \\
\hline$[\mathrm{Fe} / \mathrm{H}]$ & $-0.224 \pm 0.057$ & $0.217 \pm 0.049$ & $0.037 \pm 0.037$ & $0.194 \pm 0.060$ \\
\hline Age (Gyr) & $7.11_{-0.72}^{+0.27}$ & $7.3 \pm 1.0$ & $2.765_{-0.056}^{+0.042}$ & $2.6 \pm 2.0$ \\
\hline$A_{V}(\mathrm{mag})$ & $0.737 \pm 0.034$ & $0.048 \pm 0.011$ & $0.120 \pm 0.019$ & $0.389 \pm 0.043$ \\
\hline Distance (pc) & $519 \pm 11$ & $267.3 \pm 1.3$ & $235.4 \pm 2.3$ & $343.2 \pm 3.9$ \\
\hline
\end{tabular}

Note. The listed parameters are those determined through the joint differential evolution Markov chain analysis described in Section 3.3. For all four systems the fixedcircular-orbit model has a higher Bayesian evidence than the eccentric-orbit model. We therefore assume a fixed-circular orbit in generating the parameters listed here.

Table 9

Derived Stellar Parameters for HAT-P-62, HAT-P-63, and HAT-P-64

\begin{tabular}{lccc}
\hline \hline Parameter & $\begin{array}{c}\text { HAT-P-62 } \\
\text { Value }\end{array}$ & $\begin{array}{c}\text { HAT-P-63 } \\
\text { Value }\end{array}$ & $\begin{array}{c}\text { HAT-P-64 } \\
\text { Value }\end{array}$ \\
\hline$M_{\star}\left(M_{\odot}\right)$ & $1.023 \pm 0.020$ & $0.925 \pm 0.023$ & $1.298 \pm 0.021$ \\
$R_{\star}\left(R_{\odot}\right)$ & $1.170 \pm 0.016$ & $0.9661_{-0.0082}^{+0.010}$ & $1.735_{-0.028}^{+0.041}$ \\
$\log g_{\star}(\mathrm{cgs})$ & $4.312 \pm 0.015$ & $4.435 \pm 0.015$ & $4.072 \pm 0.015$ \\
$\rho_{\star}\left(\mathrm{g} \mathrm{cm}^{-3}\right)$ & $0.901 \pm 0.042$ & $1.448 \pm 0.061$ & $0.350 \pm 0.018$ \\
$L_{\star}\left(L_{\odot}\right)$ & $1.232 \pm 0.053$ & $0.714 \pm 0.028$ & $4.66_{-0.17}^{+0.29}$ \\
$T_{\text {eff }}(\mathrm{K})$ & $5629 \pm 48$ & $5400_{-39}^{+55}$ & $6457 \pm 29$ \\
{$[\mathrm{Fe} / \mathrm{H}]$} & $0.414 \pm 0.090$ & $0.251 \pm 0.061$ & $-0.113_{-0.056}^{+0.027}$ \\
Age $(\mathrm{Gyr})$ & $8.1 \pm 1.1$ & $9.0 \pm 1.7$ & $2.88 \pm 0.13$ \\
$A_{V}(\mathrm{mag})$ & $0.339 \pm 0.046$ & $0.506 \pm 0.046$ & $0.650_{-0.021}^{+0.014}$ \\
Distance $(\mathrm{pc})$ & $353.1 \pm 4.4$ & $408.0 \pm 4.0$ & $655_{-11}^{+17}$ \\
\hline
\end{tabular}

Note. The listed parameters are those determined through the joint differential evolution Markov chain analysis described in Section 3.3. For all three systems the fixed-circular-orbit model has a higher Bayesian evidence than the eccentric-orbit model. We therefore assume a fixed-circular orbit in generating the parameters listed here.

\section{Discussion}

We presented the discovery of seven hot Jupiters transiting bright stars. These planets were first identified as transiting planet candidates by the HATNet survey from among some six million stars that have been observed to date since 2004. They were subsequently confirmed and accurately characterized using high-precision time-series photometry from FLWO $1.2 \mathrm{~m} /$ KeplerCam, and the NASA TESS mission, and high-resolution spectroscopy, enabling high-precision RV measurements, carried out with the FLWO $1.5 \mathrm{~m} /$ TRES, Keck-I/HIRES, OHP $1.93 \mathrm{~m} /$ SOPHIE, Subaru $8 \mathrm{~m} / \mathrm{HDS}$, APO $3.5 \mathrm{~m} /$ ARCES, NOT $2.5 \mathrm{~m} /$ FIES, and LCOGT $1 \mathrm{~m} /$ NRES telescopes/instruments.

The planets discovered here contribute to the growing sample of transiting planets with precisely measured masses and radii. All seven planets have radii measured to better than $\sim 10 \%$ precision, and six of them have masses measured to this level of precision as well. Such planets are valuable contributions to the growing sample of well-characterized exoplanets which may be used in statistical studies to test theories of planet formation and evolution. In fact, the planets presented here have already been included in one such study (Hartman et al. 2016).
In Figure 5 we compare the newly discovered planets to the sample of known transiting planets with masses and radii both measured to better than $10 \%$ precision, which we take from the NASA Exoplanet Archive (accessed 2020 October 9). We show planet mass-radius, equilibrium temperature-radius, and semimajor axis-mass diagrams. As seen on these diagrams, the newly discovered planets are hot Jupiters with properties similar to the existing sample of hot Jupiters. Of particular note are the planets HAT-P-58b, HAT-P-60b, and HAT-P-64b which are hot, inflated planets that lie near the top of the distribution of planets in the mass-radius diagram. These planets have large scale heights, making them good targets for transmission spectroscopy, as we discuss further below. The other four planets are not particularly inflated, with the $M_{p}=1.057 \pm 0.070 M_{\mathrm{J}}, R_{p}=0.899 \pm 0.027 R_{\mathrm{J}}$ planet HATP-61b being actually quite compact given its mass and equilibrium temperature. This planet presumably has a large heavy-element fraction, distributed through the atmosphere of the planet, and/or condensed into a solid core (e.g., Guillot 2005). A quantitative estimate for the heavy-element fraction of HAT-P-61b would depend on theoretical models which are known to be inaccurate for planets with equilibrium temperatures above 1000 K (e.g., Fortney \& Nettelmann 2010), so we do not attempt to provide such an estimate here.

Figure 6 shows all seven host stars on $G_{\text {abs }}$ versus $B P_{0}-R P_{0}$ and $G_{\text {abs }}$ versus $G_{0}-K_{s, 0}$ CMDs. As is apparent from this diagram, the three inflated planets HAT-P-58b, HAT-P-60b and HAT-P-64b orbit host stars that are at the main-sequence turn-off, and beginning their evolution into giants. The other four host stars are all main-sequence $\mathrm{G}$ dwarfs.

Close-in giant planets transiting bright stars, such as these, can also be followed-up in a modest amount of time using current facilities to measure their orbital (mis-)alignments and probe the planetary atmospheres. We estimate that the amplitude of the Rossiter-McLaughlin effect is: $35 \mathrm{~m} \mathrm{~s}^{-1}$, $18 \mathrm{~m} \mathrm{~s}^{-1}, 36 \mathrm{~m} \mathrm{~s}^{-1}, 23 \mathrm{~m} \mathrm{~s}^{-1}, 30 \mathrm{~m} \mathrm{~s}^{-1}, 44 \mathrm{~m} \mathrm{~s}^{-1}$, and $128 \mathrm{~m} \mathrm{~s}^{-1}$, for HAT-P-58b-HAT-P-64b, respectively. Given the host star brightnesses, measured RV jitter values, and transit durations, the effect would be detectable using facilities ranging from FLWO $1.5 \mathrm{~m} /$ TRES (HAT-P-60b which orbits a $V=9.710 \pm$ 0.050 mag host star, and HAT-P-64b with its large-amplitude signal and long-lasting transits), to Keck-I/HIRES (HAT-P-59b). With $a / R_{\star}>9$, and $T_{\text {eff } \star}<6000 \mathrm{~K}$, HAT-P-59b and HAT-P-63b may be particularly interesting objects for which to observe this 
Table 10

Orbital and Planetary Parameters for HAT-P-58b, HAT-P-59b, HAT-P-60b, and HAT-P-61b

\begin{tabular}{|c|c|c|c|c|}
\hline Parameter & $\begin{array}{c}\text { HAT-P-58b } \\
\text { Value }\end{array}$ & $\begin{array}{c}\text { HAT-P-59b } \\
\text { Value }\end{array}$ & $\begin{array}{c}\text { HAT-P-60b } \\
\text { Value }\end{array}$ & $\begin{array}{c}\text { HAT-P-61b } \\
\text { Value }\end{array}$ \\
\hline \multicolumn{5}{|l|}{ Light-curve parameters } \\
\hline$P$ (days) & $4.0138379 \pm 0.0000024$ & $4.1419771 \pm 0.0000012$ & $4.7947813 \pm 0.0000024$ & $1.90231289 \pm 0.00000077$ \\
\hline$T_{c}\left(\mathrm{BJD}_{\mathrm{TDB}}-2450000\right)^{\mathrm{a}}$ & $7369.03094 \pm 0.00056$ & $8618.54088 \pm 0.00021$ & $8360.94029 \pm 0.00056$ & $7851.21119 \pm 0.00047$ \\
\hline$T_{14}(\text { days) })^{\mathrm{a}}$ & $0.1729 \pm 0.0015$ & $0.09747 \pm 0.00097$ & $0.2098 \pm 0.0015$ & $0.0691 \pm 0.0012$ \\
\hline$T_{12}=T_{34}$ (days) $^{\mathrm{a}}$ & $0.0193 \pm 0.0010$ & $0.02624 \pm 0.00099$ & $0.02557 \pm 0.00073$ & $0.01372 \pm 0.00085$ \\
\hline$a / R_{\star}$ & $7.02 \pm 0.15$ & $9.87 \pm 0.12$ & $6.146_{-0.077}^{+0.057}$ & $6.90 \pm 0.13$ \\
\hline$\zeta / R_{\star}^{\mathrm{b}}$ & $13.00 \pm 0.10$ & $26.81 \pm 0.41$ & $10.81 \pm 0.10$ & $35.46 \pm 0.88$ \\
\hline$R_{p} / R_{\star}$ & $0.0895 \pm 0.0017$ & $0.10452 \pm 0.00096$ & $0.07622 \pm 0.00055$ & $0.0984 \pm 0.0025$ \\
\hline$b^{2}$ & $0.285_{-0.032}^{+0.033}$ & $0.689_{-0.015}^{+0.013}$ & $0.446_{-0.018}^{+0.014}$ & $0.589_{-0.026}^{+0.024}$ \\
\hline$b \equiv a \cos i / R_{\star}$ & $0.534_{-0.031}^{+0.030}$ & $0.8299_{-0.0089}^{+0.0077}$ & $0.668_{-0.014}^{+0.011}$ & $0.767_{-0.017}^{+0.015}$ \\
\hline$i(\operatorname{deg})$ & $85.64 \pm 0.34$ & $85.180 \pm 0.100$ & $83.75 \pm 0.17$ & $83.62 \pm 0.24$ \\
\hline \multicolumn{5}{|l|}{ HATNet blend factors ${ }^{\mathrm{c}}$} \\
\hline Blend factor 1 & $\cdots$ & $\cdots$ & $\cdots$ & $0.87 \pm 0.10$ \\
\hline Blend factor 2 & $\cdots$ & $\ldots$ & $\ldots$ & $0.915 \pm 0.065$ \\
\hline \multicolumn{5}{|l|}{ TESS blend factors ${ }^{c}$} \\
\hline Blend factor 1 & $0.940 \pm 0.038$ & $0.997 \pm 0.012$ & $0.9957 \pm 0.0018$ & $0.694 \pm 0.040$ \\
\hline Blend factor 2 & $\ldots$ & $0.9993 \pm 0.0018$ & $\ldots$ & $\ldots$ \\
\hline Blend factor 3 & $\ldots$ & $0.9982 \pm 0.0047$ & $\ldots$ & $\ldots$ \\
\hline Blend factor 4 & $\ldots$ & $0.9989 \pm 0.0038$ & $\ldots$ & $\ldots$ \\
\hline Blend factor 5 & $\ldots$ & $0.996 \pm 0.023$ & $\ldots$ & $\ldots$ \\
\hline Blend factor 6 & $\cdots$ & $0.996 \pm 0.012$ & $\ldots$ & $\ldots$ \\
\hline Blend factor 7 & $\ldots$ & $0.9993 \pm 0.0018$ & $\ldots$ & $\cdots$ \\
\hline \multicolumn{5}{|l|}{ Limb-darkening coefficients ${ }^{\mathrm{d}}$} \\
\hline$c_{1}, R$ & $\cdots$ & $\cdots$ & $\cdots$ & $0.38 \pm 0.16$ \\
\hline$c_{2}, R$ & $\ldots$ & $\ldots$ & $\ldots$ & $0.36 \pm 0.17$ \\
\hline$c_{1}, r$ & $0.23 \pm 0.14$ & $0.43 \pm 0.16$ & $0.47 \pm 0.15$ & $0.40 \pm 0.16$ \\
\hline$c_{2}, r$ & $0.25 \pm 0.17$ & $0.36 \pm 0.17$ & $0.28 \pm 0.15$ & $0.39 \pm 0.16$ \\
\hline$c_{1}, i$ & $0.18 \pm 0.10$ & $0.32 \pm 0.14$ & $0.18 \pm 0.12$ & $0.32 \pm 0.16$ \\
\hline$c_{2}, i$ & $0.14 \pm 0.15$ & $0.21 \pm 0.15$ & $0.09 \pm 0.14$ & $0.30 \pm 0.16$ \\
\hline$c_{1}, z$ & $\ldots$ & $\ldots$ & $0.137_{-0.096}^{+0.129}$ & $\ldots$ \\
\hline$c_{2}, z$ & $\ldots$ & $\ldots$ & $0.12 \pm 0.15$ & $\ldots$ \\
\hline$c_{1}, T$ & $0.26 \pm 0.13$ & $0.16 \pm 0.11$ & $0.19 \pm 0.11$ & $0.31 \pm 0.14$ \\
\hline$c_{2}, T$ & $0.24 \pm 0.16$ & $0.29 \pm 0.14$ & $0.23 \pm 0.15$ & $0.29 \pm 0.16$ \\
\hline \multicolumn{5}{|l|}{ RV parameters } \\
\hline$K\left(\mathrm{~m} \mathrm{~s}^{-1}\right)$ & $46.4 \pm 3.6$ & $192.6 \pm 7.7$ & $54.1 \pm 3.5$ & $173 \pm 11$ \\
\hline$e^{\mathrm{e}}$ & $<0.073$ & $<0.030$ & $<0.250$ & $<0.113$ \\
\hline RV jitter HIRES $\left(\mathrm{m} \mathrm{s}^{-1}\right)^{\mathrm{f}}$ & $<12.6$ & $\ldots$ & $12.3 \pm 3.7$ & $<61.3$ \\
\hline RV jitter TRES $\left(\mathrm{m} \mathrm{s}^{-1}\right)$ & $\cdots$ & $<38.4$ & $<12.4$ & $39 \pm 11$ \\
\hline RV jitter SOPHIE $\left(\mathrm{m} \mathrm{s}^{-1}\right)$ & $\ldots$ & $<17.6$ & $<15.0$ & $\ldots$ \\
\hline RV jitter NRES/ELP $\left(\mathrm{m} \mathrm{s}^{-1}\right)$ & $\ldots$ & $\ldots$ & $56 \pm 13$ & $\ldots$ \\
\hline RV jitter NRES/TLV $\left(\mathrm{m} \mathrm{s}^{-1}\right)$ & $\ldots$ & $\cdots$ & $22 \pm 19$ & $\ldots$ \\
\hline \multicolumn{5}{|l|}{ Planetary parameters } \\
\hline$M_{p}\left(M_{\mathrm{J}}\right)$ & $0.372 \pm 0.030$ & $1.540 \pm 0.067$ & $0.574 \pm 0.038$ & $1.057 \pm 0.070$ \\
\hline$R_{p}\left(R_{\mathrm{J}}\right)$ & $1.332 \pm 0.043$ & $1.123 \pm 0.013$ & $1.631 \pm 0.024$ & $0.899 \pm 0.027$ \\
\hline$C\left(M_{p}, R_{p}\right)^{\mathrm{g}}$ & 0.02 & -0.16 & -0.02 & -0.04 \\
\hline$\rho_{p}\left(\mathrm{~g} \mathrm{~cm}^{-3}\right)$ & $0.194 \pm 0.024$ & $1.347 \pm 0.081$ & $0.164 \pm 0.013$ & $1.80 \pm 0.20$ \\
\hline $\log g_{p}(\mathrm{cgs})$ & $2.714 \pm 0.045$ & $3.481 \pm 0.023$ & $2.730 \pm 0.032$ & $3.510 \pm 0.040$ \\
\hline$a(\mathrm{au})$ & $0.04994 \pm 0.00044$ & $0.05064 \pm 0.00037$ & $0.06277 \pm 0.00017$ & $0.03010 \pm 0.00034$ \\
\hline$T_{\text {eq }}(\mathrm{K})$ & $1622 \pm 18$ & $1277.8 \pm 6.5$ & $1772 \pm 12$ & $1505 \pm 16$ \\
\hline$\Theta^{\mathrm{h}}$ & $0.0269 \pm 0.0023$ & $0.1367 \pm 0.0058$ & $0.0306 \pm 0.0020$ & $0.0702 \pm 0.0047$ \\
\hline $\log _{10}\langle F\rangle(\operatorname{cgs})^{\mathrm{i}}$ & $9.193 \pm 0.019$ & $8.7787 \pm 0.0088$ & $9.347 \pm 0.012$ & $9.063 \pm 0.018$ \\
\hline
\end{tabular}

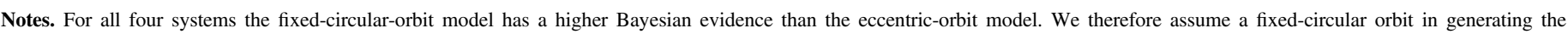
parameters listed here.

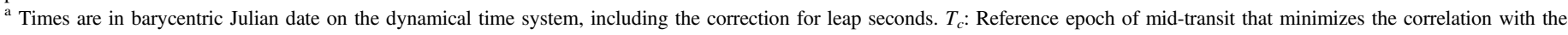
orbital period. $T_{14}$ : total transit duration, time between first to last contact; $T_{12}=T_{34}$ : ingress/egress time, time between first and second, or third and fourth contact.

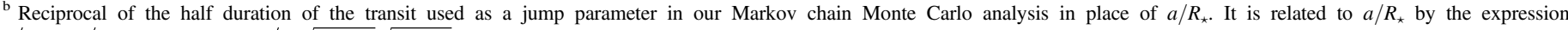
$\zeta / R_{\star}=a / R_{\star}(2 \pi(1+e \sin \omega)) /\left(P \sqrt{1-b^{2}} \sqrt{1-e^{2}}\right)$ (Bakos et al. 2010).

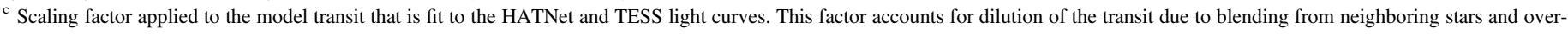

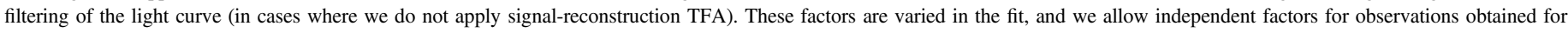

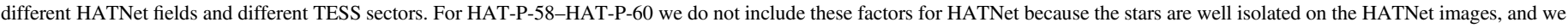
apply signal-reconstruction TFA to preserve the signal shape while filtering the light curves.

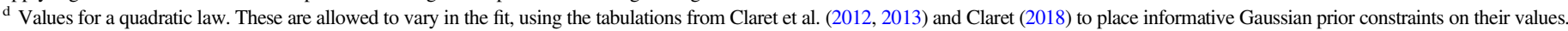

e The $95 \%$ confidence upper limit on the eccentricity determined when $\sqrt{e} \cos \omega$ and $\sqrt{e} \sin \omega$ are allowed to vary in the fit.

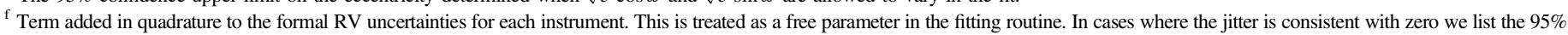
confidence upper limit.

${ }^{\mathrm{g}}$ Correlation coefficient between the planetary mass $M_{p}$ and radius $R_{p}$ estimated from the posterior parameter distribution.

h The Safronov number is given by $\Theta=\frac{1}{2}\left(V_{\text {esc }} / V_{\text {orb }}\right)^{2}=\left(a / R_{p}\right)\left(M_{p} / M_{\star}\right)$ (see Hansen \& Barman 2007).

${ }^{\mathrm{i}}$ Incoming flux per unit surface area, averaged over the orbit. 
Table 11

Orbital and Planetary Parameters for HAT-P-62b, HAT-P-63b, and HAT-P-64b

\begin{tabular}{|c|c|c|c|}
\hline Parameter & $\begin{array}{l}\text { HAT-P-62b } \\
\text { Value }\end{array}$ & $\begin{array}{l}\text { HAT-P-63b } \\
\text { Value }\end{array}$ & $\begin{array}{l}\text { HAT-P-64b } \\
\text { Value }\end{array}$ \\
\hline \multicolumn{4}{|l|}{ Light-curve parameters } \\
\hline$P$ (days) & $2.6453235 \pm 0.0000039$ & $3.377728 \pm 0.000013$ & $4.0072320 \pm 0.0000017$ \\
\hline$T_{c}\left(\mathrm{BJD}_{\mathrm{TDB}}-2450000\right)^{\mathrm{a}}$ & $7118.38979 \pm 0.00044$ & $6382.94256 \pm 0.00053$ & $7751.46354 \pm 0.00063$ \\
\hline$T_{14}(\text { days) })^{\mathrm{a}}$ & $0.1293 \pm 0.0012$ & $0.1222 \pm 0.0016$ & $0.2052 \pm 0.0020$ \\
\hline$T_{12}=T_{34}(\text { days })^{\mathrm{a}}$ & $0.01183 \pm 0.00050$ & $0.01392 \pm 0.00058$ & $0.0199 \pm 0.0010$ \\
\hline$a / R_{\star}$ & $6.93 \pm 0.11$ & $9.56 \pm 0.14$ & $6.67 \pm 0.12$ \\
\hline$\zeta / R_{\star}^{\mathrm{b}}$ & $17.02 \pm 0.17$ & $18.45 \pm 0.27$ & $10.79 \pm 0.11$ \\
\hline$R_{p} / R_{\star}$ & $0.0942 \pm 0.0019$ & $0.1191 \pm 0.0032$ & $0.1007 \pm 0.0034$ \\
\hline$b^{2}$ & $0.063_{-0.035}^{+0.036}$ & $0.069_{-0.030}^{+0.040}$ & $0.054_{-0.030}^{+0.046}$ \\
\hline$b \equiv a \cos i / R_{\star}$ & $0.250_{-0.084}^{+0.064}$ & $0.262_{-0.066}^{+0.068}$ & $0.232_{-0.079}^{+0.085}$ \\
\hline$i(\mathrm{deg})$ & $87.93 \pm 0.64$ & $88.45 \pm 0.44$ & $88.01 \pm 0.70$ \\
\hline \multicolumn{4}{|l|}{ HATNet blend factors ${ }^{\mathrm{c}}$} \\
\hline Blend factor & $0.839 \pm 0.055$ & $\ldots$ & $0.748 \pm 0.072$ \\
\hline \multicolumn{4}{|l|}{ TESS blend factors ${ }^{\mathrm{c}}$} \\
\hline Blend factor & $\cdots$ & $\cdots$ & $0.871 \pm 0.062$ \\
\hline \multicolumn{4}{|l|}{ Limb-darkening coefficients ${ }^{\mathrm{d}}$} \\
\hline$c_{1}, r$ & $0.36 \pm 0.15$ & $0.44 \pm 0.15$ & $0.26 \pm 0.14$ \\
\hline$c_{2}, r$ & $0.31 \pm 0.18$ & $0.38 \pm 0.16$ & $0.26 \pm 0.17$ \\
\hline$c_{1}, i$ & $0.33 \pm 0.10$ & $0.47 \pm 0.13$ & $0.26 \pm 0.14$ \\
\hline$c_{2}, i$ & $0.22 \pm 0.16$ & $0.41 \pm 0.14$ & $0.27 \pm 0.17$ \\
\hline$c_{1}, T$ & $\ldots$ & $\ldots$ & $0.29 \pm 0.11$ \\
\hline$c_{2}, T$ & $\cdots$ & $\cdots$ & $0.37 \pm 0.16$ \\
\hline \multicolumn{4}{|l|}{ RV parameters } \\
\hline$K\left(\mathrm{~m} \mathrm{~s}^{-1}\right)$ & $110 \pm 13$ & $87.3 \pm 3.2$ & $62 \pm 18$ \\
\hline$e^{\mathrm{e}}$ & $<0.101$ & $<0.069$ & $<0.101$ \\
\hline RV jitter HIRES $\left(\mathrm{m} \mathrm{s}^{-1}\right)^{\mathrm{f}}$ & $\ldots$ & $\ldots$ & $21 \pm 10$ \\
\hline RV jitter TRES $\left(\mathrm{m} \mathrm{s}^{-1}\right)$ & $33 \pm 11$ & $<1.9$ & $\ldots$ \\
\hline RV jitter SOPHIE $\left(\mathrm{m} \mathrm{s}^{-1}\right)$ & $\ldots$ & $16 \pm 10$ & $<69.2$ \\
\hline $\mathrm{RV}$ jitter HDS $\left(\mathrm{m} \mathrm{s}^{-1}\right)$ & $\ldots$ & $<2.4$ & $\ldots$ \\
\hline \multicolumn{4}{|l|}{ Planetary parameters } \\
\hline$M_{p}\left(M_{\mathrm{J}}\right)$ & $0.761 \pm 0.088$ & $0.614 \pm 0.024$ & $0.58_{-0.13}^{+0.18}$ \\
\hline$R_{p}\left(R_{\mathrm{J}}\right)$ & $1.073 \pm 0.029$ & $1.119 \pm 0.033$ & $1.703 \pm 0.070$ \\
\hline$C\left(M_{p}, R_{p}\right)^{\mathrm{g}}$ & 0.02 & -0.25 & 0.06 \\
\hline$\rho_{p}\left(\mathrm{~g} \mathrm{~cm}^{-3}\right)$ & $0.77 \pm 0.11$ & $0.540 \pm 0.055$ & $0.144_{-0.035}^{+0.046}$ \\
\hline $\log g_{p}(\mathrm{cgs})$ & $3.214 \pm 0.056$ & $3.082 \pm 0.034$ & $2.69 \pm 0.12$ \\
\hline$a(\mathrm{au})$ & $0.03772 \pm 0.00024$ & $0.04294 \pm 0.00035$ & $0.05387 \pm 0.00030$ \\
\hline$T_{\mathrm{eq}}(\mathrm{K})$ & $1512 \pm 13$ & $1237 \pm 11$ & $1766_{-16}^{+22}$ \\
\hline$\Theta^{\mathrm{h}}$ & $0.0522 \pm 0.0061$ & $0.0506 \pm 0.0026$ & $0.0281_{-0.0064}^{+0.0084}$ \\
\hline $\log _{10}\langle F\rangle(\operatorname{cgs})^{\mathrm{i}}$ & $9.072 \pm 0.015$ & $8.722 \pm 0.015$ & $9.341_{-0.016}^{+0.0021}$ \\
\hline
\end{tabular}

Note. For all three systems the fixed-circular-orbit model has a higher Bayesian evidence than the eccentric-orbit model. We therefore assume a fixed-circular orbit in generating the parameters listed here. For all further tablenotes refer to Table 10.

effect, in an effort to determine whether giant planets transiting cool stars become less well aligned as the strength of the tidal interaction with their host stars decreases (e.g., Albrecht et al. 2012).

As regards atmospheric characterization, with its $1 \%$ deep transits lasting almost five hours, and large atmospheric scale height $\left(\log g_{p}=2.69 \pm 0.12\right)$, HAT-P-64b is perhaps the most promising of the planets discovered here for having readily detectable features in its transmission spectrum. These may be atomic or molecular absorption features as seen, for example, in the spectrum of the inflated Neptune HAT-P-26b, (Wakeford et al. 2017), among many other planets. Alternatively, this may be evidence of an atmospheric haze revealed through Rayleigh scattering, as seen, for example, in the spectrum of the highly inflated hot Jupiter HAT-P-32b, (Mallonn \& Wakeford 2017), again among many planets. The bottom-right panel of Figure 5 compares the ease of transmission spectrum observations for the newly discovered transiting planets to the known sample of planets with precisely measured masses and radii. Here we estimate the relative expected transmission spectrum observation time by assuming the transmission spectrum signal scales as $H R_{p} / R_{\star}^{2}$ (e.g., Miller-Ricci et al. 2009), and assume $K_{s}$-band for determining the expected flux and associated photometric Poisson noise. As shown in this diagram, HAT-P-58b, HAT-P$60 \mathrm{~b}$, and HAT-P-64b all are promising targets for transmission spectroscopy by this measure. These planets could be studied with existing ground- and space-based facilities, and are also good targets for upcoming facilities such as the James Webb Space Telescope and extremely large ground-based telescopes that are under construction.

With a planetary radius of $1.703 \pm 0.070 R_{\mathrm{J}}$, HAT-P-64b is also one of the largest known transiting exoplanets (as of 2018 July there are only 23 transiting planets listed in the NASA exoplanet archive with larger radii). The planet follows the 
Table 12

Relative Radial Velocities and Bisector Spans for HAT-P-58-HAT-P-64

\begin{tabular}{|c|c|c|c|c|c|c|c|c|}
\hline Star & $\begin{array}{l}\text { BJD } \\
(2,450,000+)\end{array}$ & $\begin{array}{c}\mathrm{RV}^{\mathrm{a}} \\
\left(\mathrm{m} \mathrm{s}^{-1}\right)\end{array}$ & $\begin{array}{c}\sigma_{\mathrm{RV}^{\mathrm{b}}}^{\mathrm{b}} \\
\left(\mathrm{m} \mathrm{s}^{-1}\right)\end{array}$ & $\begin{array}{c}\mathrm{BS} \\
\left(\mathrm{m} \mathrm{s}^{-1}\right)\end{array}$ & $\begin{array}{c}\sigma_{\mathrm{BS}} \\
\left(\mathrm{m} \mathrm{s}^{-1}\right)\end{array}$ & $\mathrm{S}_{\mathrm{HK}}{ }^{\mathrm{c}}$ & Phase & Instrument \\
\hline \multicolumn{9}{|c|}{ HAT-P-58 } \\
\hline HAT-P-58 & 6890.12499 & 40.53 & 7.42 & 33.5 & 20.2 & 0.172 & 0.686 & HIRES \\
\hline HAT-P-58 & 6892.12712 & -46.48 & 2.99 & -16.9 & 9.8 & 0.153 & 0.185 & HIRES \\
\hline HAT-P-58 & 6894.09295 & 36.41 & 2.71 & -4.2 & 3.1 & 0.144 & 0.675 & HIRES \\
\hline HAT-P-58 & 6895.09290 & $\ldots$ & $\ldots$ & -15.5 & 4.7 & 0.145 & 0.924 & HIRES \\
\hline HAT-P-58 & 6896.07796 & -37.36 & 2.87 & -14.6 & 5.6 & 0.142 & 0.169 & HIRES \\
\hline HAT-P-58 & 6909.10189 & -20.66 & 3.83 & 13.6 & 15.5 & 0.157 & 0.414 & HIRES \\
\hline HAT-P-58 & 6910.07872 & 54.59 & 4.93 & 24.4 & 48.1 & 0.145 & 0.657 & HIRES \\
\hline HAT-P-58 & 6912.08980 & -36.75 & 2.87 & -20.4 & 8.0 & 0.143 & 0.158 & HIRES \\
\hline \multicolumn{9}{|l|}{ HAT-P-59 } \\
\hline HAT-P-59 & 6581.71540 & -173.34 & 30.32 & -8.1 & 16.1 & $\ldots$ & 0.248 & TRES \\
\hline HAT-P-59 & 6583.69510 & 222.45 & 26.21 & 14.5 & 9.7 & $\ldots$ & 0.726 & TRES \\
\hline HAT-P-59 & 6585.65905 & -166.02 & 31.64 & 4.4 & 9.3 & $\ldots$ & 0.200 & TRES \\
\hline HAT-P-59 & 6593.29170 & -42.34 & 11.50 & -48.7 & 22.9 & $\ldots$ & 0.043 & SOPHIE \\
\hline HAT-P-59 & 6595.25025 & 51.96 & 36.10 & -136.2 & 72.2 & $\ldots$ & 0.516 & SOPHIE \\
\hline HАТ-Р-59 & 6596.25559 & 214.46 & 14.70 & -12.8 & 29.5 & $\ldots$ & 0.759 & SOPHIE \\
\hline HAT-P-59 & 6597.24301 & -6.84 & 9.70 & -44.5 & 19.5 & $\ldots$ & 0.997 & SOPHIE \\
\hline HAT-P-59 & 6598.26817 & -164.04 & 31.80 & -50.0 & 63.6 & $\ldots$ & 0.244 & SOPHIE \\
\hline HAT-P-59 & 6599.25285 & -31.24 & 12.00 & -22.5 & 24.0 & $\ldots$ & 0.482 & SOPHIE \\
\hline HAT-P-59 & 6599.26717 & -32.54 & 21.10 & -81.7 & 42.1 & $\ldots$ & 0.486 & SOPHIE \\
\hline HAT-P-59 & 6600.23567 & 218.06 & 21.10 & 46.2 & 42.2 & $\ldots$ & 0.719 & SOPHIE \\
\hline HAT-P-59 & 6601.31614 & 27.36 & 9.30 & -29.2 & 18.5 & $\ldots$ & 0.980 & SOPHIE \\
\hline HAT-P-59 & 6602.30141 & -195.44 & 14.40 & -15.3 & 28.8 & $\ldots$ & 0.218 & SOPHIE \\
\hline HAT-P-59 & 6605.60024 & -49.83 & 19.04 & -27.6 & 7.9 & $\ldots$ & 0.015 & TRES \\
\hline HAT-P-59 & 6606.60413 & -152.84 & 24.81 & -3.4 & 24.4 & $\ldots$ & 0.257 & TRES \\
\hline HAT-P-59 & 6607.59892 & -19.99 & 19.87 & 0.8 & 11.9 & $\ldots$ & 0.497 & TRES \\
\hline HAT-P-59 & 6608.60682 & 190.72 & 31.37 & -4.0 & 13.8 & $\ldots$ & 0.740 & TRES \\
\hline HAT-P-59 & 6609.61035 & 27.93 & 18.27 & 31.0 & 13.6 & $\ldots$ & 0.983 & TRES \\
\hline HAT-P-59 & 6610.62724 & -214.20 & 26.99 & 23.6 & 10.3 & $\ldots$ & 0.228 & TRES \\
\hline HAT-P-59 & 6611.59706 & -3.09 & 21.75 & 18.0 & 17.5 & $\ldots$ & 0.462 & TRES \\
\hline HAT-P-59 & 6615.58195 & -114.55 & 21.68 & -1.0 & 9.4 & $\ldots$ & 0.425 & TRES \\
\hline HAT-P-59 & 6616.59129 & 144.92 & 12.85 & -19.8 & 11.0 & $\ldots$ & 0.668 & TRES \\
\hline HAT-P-59 & 6617.62764 & 62.24 & 12.85 & -28.4 & 10.4 & $\cdots$ & 0.918 & TRES \\
\hline \multicolumn{9}{|l|}{ HAT-P-60 } \\
\hline HAT-P-60 & 6326.58810 & 42.15 & 24.93 & -18.2 & 15.9 & $\ldots$ & 0.716 & TRES \\
\hline HAT-P-60 & 6549.78555 & -57.10 & 47.30 & 24.4 & 31.6 & $\ldots$ & 0.266 & TRES \\
\hline HAT-P-60 & 6551.82293 & 23.30 & 36.39 & 44.7 & 35.9 & $\ldots$ & 0.691 & TRES \\
\hline HAT-P-60 & 6558.92278 & -32.08 & 34.84 & -51.5 & 28.6 & $\ldots$ & 0.172 & TRES \\
\hline HAT-P-60 & 6573.89597 & -44.23 & 19.98 & 15.3 & 10.0 & $\ldots$ & 0.295 & TRES \\
\hline НАТ-Р-60 & 6574.90009 & -41.76 & 24.66 & 4.4 & 14.1 & $\ldots$ & 0.504 & TRES \\
\hline HAT-P-60 & 6575.79583 & 38.08 & 23.17 & -16.4 & 15.2 & $\ldots$ & 0.691 & TRES \\
\hline HAT-P-60 & 6576.77948 & 9.39 & 24.46 & -1.6 & 17.9 & $\ldots$ & 0.896 & TRES \\
\hline HAT-P-60 & 6577.80537 & -33.50 & 24.93 & -3.6 & 31.4 & $\ldots$ & 0.110 & TRES \\
\hline HAT-P-60 & 6578.74876 & -61.26 & 18.25 & -7.1 & 14.2 & $\ldots$ & 0.307 & TRES \\
\hline HAT-P-60 & 6580.72123 & 66.89 & 18.25 & 5.2 & 14.7 & $\ldots$ & 0.718 & TRES \\
\hline HAT-P-60 & 6581.79896 & 32.21 & 21.62 & 13.8 & 14.6 & $\ldots$ & 0.943 & TRES \\
\hline HAT-P-60 & 6582.85746 & -63.67 & 21.79 & -9.5 & 11.2 & $\cdots$ & 0.164 & TRES \\
\hline HAT-P-60 & 6593.49292 & -16.13 & 32.40 & -82.7 & 64.8 & $\ldots$ & 0.382 & SOPHIE \\
\hline HAT-P-60 & 6595.51401 & 32.07 & 27.10 & -54.7 & 54.1 & $\ldots$ & 0.803 & SOPHIE \\
\hline HAT-P-60 & 6596.66289 & -15.03 & 22.50 & 28.5 & 45.0 & $\ldots$ & 0.043 & SOPHIE \\
\hline НАТ-Р-60 & 6597.40379 & -27.23 & 22.30 & -23.5 & 44.6 & $\ldots$ & 0.197 & SOPHIE \\
\hline HAT-P-60 & 6599.55850 & 35.07 & 14.40 & -74.3 & 28.8 & $\ldots$ & 0.647 & SOPHIE \\
\hline HAT-P-60 & 6600.53433 & 45.07 & 26.20 & -38.2 & 52.3 & $\ldots$ & 0.850 & SOPHIE \\
\hline HAT-P-60 & 6601.63510 & -28.93 & 22.50 & 15.5 & 45.0 & $\ldots$ & 0.080 & SOPHIE \\
\hline HAT-P-60 & 6602.40299 & -67.13 & 15.10 & -10.2 & 30.2 & $\ldots$ & 0.240 & SOPHIE \\
\hline HAT-P-60 & 6637.75333 & 29.51 & 3.22 & 6.3 & 3.2 & 0.121 & 0.613 & HIRES \\
\hline HAT-P-60 & 6638.81890 & 33.03 & 4.25 & 0.3 & 2.8 & 0.123 & 0.835 & HIRES \\
\hline HAT-P-60 & 7353.80988 & 36.33 & 4.11 & -8.1 & 5.4 & 0.124 & 0.954 & HIRES \\
\hline HAT-P-60 & 7354.79800 & -40.93 & 3.99 & 8.4 & 4.5 & 0.124 & 0.160 & HIRES \\
\hline HAT-P-60 & 7355.79193 & -21.46 & 3.78 & -0.8 & 3.7 & 0.123 & 0.367 & HIRES \\
\hline
\end{tabular}


Table 12

(Continued)

\begin{tabular}{|c|c|c|c|c|c|c|c|c|}
\hline Star & $\begin{array}{l}\text { BJD } \\
(2,450,000+)\end{array}$ & $\begin{array}{c}\mathrm{RV}^{\mathrm{a}} \\
\left(\mathrm{m} \mathrm{s}^{-1}\right)\end{array}$ & $\begin{array}{c}\sigma_{\mathrm{RV}^{\mathrm{b}}} \\
\left(\mathrm{m} \mathrm{s}^{-1}\right)\end{array}$ & $\begin{array}{c}\mathrm{BS} \\
\left(\mathrm{m} \mathrm{s}^{-1}\right)\end{array}$ & $\begin{array}{c}\sigma_{\mathrm{BS}} \\
\left(\mathrm{m} \mathrm{s}^{-1}\right)\end{array}$ & $\mathrm{S}_{\mathrm{HK}}{ }^{\mathrm{c}}$ & Phase & Instrument \\
\hline HAT-P-60 & 7355.89024 & $\ldots$ & $\ldots$ & -7.1 & 4.1 & 0.122 & 0.387 & HIRES \\
\hline HAT-P-60 & 7378.72938 & -26.21 & 4.10 & -0.3 & 3.6 & 0.126 & 0.151 & HIRES \\
\hline HAT-P-60 & 7401.88232 & 22.14 & 4.50 & 10.4 & 4.5 & 0.121 & 0.980 & HIRES \\
\hline HAT-P-60 & 7412.79458 & -58.33 & 4.40 & -9.1 & 3.5 & 0.128 & 0.255 & HIRES \\
\hline \multicolumn{9}{|l|}{ HAT-P-61 } \\
\hline HAT-P-61 & 6910.98025 & 149.18 & 26.04 & 76.1 & 25.9 & $\ldots$ & 0.743 & TRES \\
\hline HAT-P-61 & 6911.92592 & -134.70 & 33.23 & 64.2 & 42.9 & $\cdots$ & 0.241 & TRES \\
\hline HAT-P-61 & 6912.96888 & 129.17 & 17.84 & 2.4 & 28.1 & $\ldots$ & 0.789 & TRES \\
\hline HAT-P-61 & 6931.91154 & 176.60 & 27.60 & -32.9 & 22.0 & $\ldots$ & 0.747 & TRES \\
\hline HAT-P-61 & 6932.88321 & -72.55 & 36.71 & -27.9 & 78.9 & $\ldots$ & 0.257 & TRES \\
\hline HAT-P-61 & 6934.95150 & -208.83 & 17.84 & -47.2 & 37.0 & $\ldots$ & 0.345 & TRES \\
\hline HAT-P-61 & 6935.94714 & 111.18 & 31.50 & -108.5 & 29.2 & $\ldots$ & 0.868 & TRES \\
\hline HAT-P-61 & 6944.99515 & 149.97 & 22.95 & 19.2 & 24.9 & $\ldots$ & 0.624 & TRES \\
\hline HAT-P-61 & 6945.92201 & -161.35 & 32.35 & 3.7 & 54.5 & $\ldots$ & 0.112 & TRES \\
\hline HAT-P-61 & 6958.94233 & 25.21 & 19.23 & 3.0 & 24.8 & $\ldots$ & 0.956 & TRES \\
\hline HAT-P-61 & 6960.00651 & 2.01 & 29.22 & -22.6 & 39.3 & $\ldots$ & 0.515 & TRES \\
\hline HAT-P-61 & 6960.95084 & -69.03 & 36.34 & -68.0 & 42.7 & $\ldots$ & 0.012 & TRES \\
\hline HAT-P-61 & 6961.93882 & 73.86 & 35.83 & 11.5 & 41.9 & $\ldots$ & 0.531 & TRES \\
\hline HAT-P-61 & 6965.93914 & 146.95 & 20.19 & -15.7 & 19.3 & $\ldots$ & 0.634 & TRES \\
\hline HAT-P-61 & 6970.92528 & -64.93 & 35.63 & 90.8 & 23.4 & $\ldots$ & 0.255 & TRES \\
\hline HAT-P-61 & 6971.87773 & 232.02 & 31.09 & 76.8 & 42.3 & $\ldots$ & 0.756 & TRES \\
\hline HAT-P-61 & 6973.00690 & -92.82 & 40.13 & -9.2 & 32.6 & $\ldots$ & 0.349 & TRES \\
\hline HAT-P-61 & 6978.94963 & -64.74 & 25.97 & -15.7 & 28.7 & $\ldots$ & 0.473 & TRES \\
\hline HAT-P-61 & 7354.08818 & 169.27 & 1.12 & 6.1 & 8.8 & 0.258 & 0.675 & HIRES \\
\hline HAT-P-61 & 7355.08733 & -171.23 & 0.90 & -1.5 & 2.5 & 0.232 & 0.200 & HIRES \\
\hline HAT-P-61 & 7356.00551 & $\ldots$ & $\ldots$ & 2.9 & 4.8 & 0.232 & 0.682 & HIRES \\
\hline HAT-P-61 & 7356.11208 & 170.17 & 0.86 & -7.5 & 4.7 & 0.237 & 0.738 & HIRES \\
\hline \multicolumn{9}{|l|}{ HAT-P-62 } \\
\hline HAT-P-62 & 6674.65588 & -126.65 & 21.61 & 30.9 & 29.2 & $\ldots$ & 0.258 & TRES \\
\hline HAT-P-62 & 6707.64331 & 88.60 & 25.94 & -13.4 & 27.3 & $\ldots$ & 0.728 & TRES \\
\hline HAT-P-62 & 6942.95953 & 148.33 & 23.82 & -47.5 & 32.3 & $\ldots$ & 0.683 & TRES \\
\hline HAT-P-62 & 6945.86085 & 164.72 & 17.63 & 11.1 & 34.1 & $\ldots$ & 0.780 & TRES \\
\hline HAT-P-62 & 6958.92253 & 74.01 & 19.07 & -15.2 & 18.8 & $\ldots$ & 0.718 & TRES \\
\hline HAT-P-62 & 6959.99100 & -30.23 & 26.36 & 1.3 & 27.9 & $\ldots$ & 0.121 & TRES \\
\hline HAT-P-62 & 6960.93014 & -1.99 & 20.56 & -15.8 & 20.6 & $\ldots$ & 0.476 & TRES \\
\hline HAT-P-62 & 6961.91630 & 33.97 & 16.43 & 24.2 & 21.0 & $\ldots$ & 0.849 & TRES \\
\hline HAT-P-62 & 6962.86334 & -108.71 & 17.73 & 52.5 & 24.9 & $\ldots$ & 0.207 & TRES \\
\hline HAT-P-62 & 6965.87438 & -126.58 & 14.60 & 0.6 & 24.2 & $\ldots$ & 0.346 & TRES \\
\hline HAT-P-62 & 6970.87354 & -45.58 & 22.84 & -75.3 & 48.3 & $\ldots$ & 0.235 & TRES \\
\hline HAT-P-62 & 6971.83494 & 56.80 & 18.00 & 5.3 & 24.7 & $\ldots$ & 0.599 & TRES \\
\hline HAT-P-62 & 6972.87045 & 2.71 & 23.65 & -30.3 & 23.0 & $\ldots$ & 0.990 & TRES \\
\hline HAT-P-62 & 6977.88426 & 84.76 & 27.25 & 52.8 & 26.9 & $\ldots$ & 0.886 & TRES \\
\hline HAT-P-62 & 6978.92626 & -134.32 & 14.60 & 18.9 & 25.0 & $\ldots$ & 0.279 & TRES \\
\hline \multicolumn{9}{|l|}{ HAT-P-63 } \\
\hline HAT-P-63 & 6023.91391 & 129.50 & 38.60 & $\ldots$ & $\ldots$ & $\ldots$ & 0.707 & TRES \\
\hline HAT-P-63 & 6045.89542 & -84.12 & 38.60 & $\ldots$ & $\ldots$ & $\ldots$ & 0.215 & TRES \\
\hline HAT-P-63 & 6189.73983 & $\ldots$ & $\ldots$ & -4.9 & 19.9 & $\ldots$ & 0.801 & HDS \\
\hline HAT-P-63 & 6189.75456 & $\ldots$ & $\ldots$ & -9.9 & 20.4 & $\ldots$ & 0.805 & HDS \\
\hline HAT-P-63 & 6189.76928 & $\ldots$ & $\ldots$ & -5.0 & 22.9 & $\ldots$ & 0.810 & HDS \\
\hline HAT-P-63 & 6189.78400 & $\ldots$ & $\ldots$ & -9.1 & 22.6 & $\ldots$ & 0.814 & HDS \\
\hline HAT-P-63 & 6190.73339 & -54.82 & 7.80 & -1.0 & 23.4 & $\ldots$ & 0.095 & HDS \\
\hline HAT-P-63 & 6190.74813 & -54.06 & 7.84 & 13.8 & 18.3 & $\ldots$ & 0.100 & HDS \\
\hline HAT-P-63 & 6190.76286 & -58.71 & 8.10 & 10.1 & 16.5 & $\ldots$ & 0.104 & HDS \\
\hline HAT-P-63 & 6190.77762 & -58.26 & 7.29 & 4.0 & 17.5 & $\ldots$ & 0.108 & HDS \\
\hline HAT-P-63 & 6191.73872 & -53.97 & 12.13 & 11.2 & 8.7 & $\ldots$ & 0.393 & HDS \\
\hline HAT-P-63 & 6191.75344 & -47.95 & 10.48 & -7.7 & 9.0 & $\ldots$ & 0.397 & HDS \\
\hline HAT-P-63 & 6191.76817 & -49.52 & 13.25 & 15.8 & 12.8 & $\ldots$ & 0.402 & HDS \\
\hline HAT-P-63 & 6191.78289 & -62.43 & 12.27 & 32.1 & 14.5 & $\ldots$ & 0.406 & HDS \\
\hline HAT-P-63 & 6192.73493 & 79.05 & 8.31 & -14.2 & 18.9 & $\ldots$ & 0.688 & HDS \\
\hline
\end{tabular}


Table 12

(Continued)

\begin{tabular}{|c|c|c|c|c|c|c|c|c|}
\hline Star & $\begin{array}{l}\text { BJD } \\
(2,450,000+)\end{array}$ & $\begin{array}{c}\mathrm{RV}^{\mathrm{a}} \\
\left(\mathrm{m} \mathrm{s}^{-1}\right)\end{array}$ & $\begin{array}{c}\sigma_{\mathrm{RV}^{\mathrm{b}}} \\
\left(\mathrm{m} \mathrm{s}^{-1}\right)\end{array}$ & $\begin{array}{c}\mathrm{BS} \\
\left(\mathrm{m} \mathrm{s}^{-1}\right)\end{array}$ & $\begin{array}{c}\sigma_{\mathrm{BS}} \\
\left(\mathrm{m} \mathrm{s}^{-1}\right)\end{array}$ & $\mathrm{S}_{\mathrm{HK}}{ }^{\mathrm{c}}$ & Phase & Instrument \\
\hline HAT-P-63 & 6192.74967 & 80.64 & 7.60 & -4.2 & 12.3 & $\ldots$ & 0.692 & HDS \\
\hline HAT-P-63 & 6192.76439 & 80.82 & 8.44 & -16.0 & 17.3 & $\ldots$ & 0.697 & HDS \\
\hline HАТ-P-63 & 6192.77911 & 77.11 & 8.65 & -14.9 & 15.3 & $\ldots$ & 0.701 & HDS \\
\hline HAT-P-63 & 6446.51381 & 54.19 & 18.60 & -51.2 & 37.2 & $\ldots$ & 0.821 & SOPHIE \\
\hline HАТ-P-63 & 6447.50510 & -35.01 & 12.50 & -6.5 & 25.0 & $\ldots$ & 0.114 & SOPHIE \\
\hline HAT-P-63 & 6448.56434 & -37.31 & 13.10 & -5.8 & 26.2 & $\ldots$ & 0.428 & SOPHIE \\
\hline HAT-P-63 & 6449.56056 & 45.69 & 14.70 & -36.7 & 29.4 & $\ldots$ & 0.723 & SOPHIE \\
\hline HAT-P-63 & 6451.51641 & -100.41 & 11.30 & -15.0 & 22.6 & $\ldots$ & 0.302 & SOPHIE \\
\hline HАТ-P-63 & 6454.53652 & -72.61 & 12.20 & -77.2 & 24.4 & $\ldots$ & 0.196 & SOPHIE \\
\hline HAT-P-63 & 6456.50551 & 103.69 & 8.20 & -21.7 & 16.4 & $\ldots$ & 0.779 & SOPHIE \\
\hline \multicolumn{9}{|l|}{ HAT-P-64 } \\
\hline HAT-P-64 & 5611.85377 & $\ldots$ & $\ldots$ & 13.3 & 8.1 & 0.159 & 0.063 & HIRES \\
\hline HAТ-P-64 & 5611.86923 & -27.85 & 8.60 & 3.8 & 8.4 & 0.140 & 0.067 & HIRES \\
\hline HАТ-P-64 & 5815.07604 & 51.41 & 6.24 & -0.1 & 8.1 & 0.141 & 0.777 & HIRES \\
\hline HAT-P-64 & 5815.08975 & $\ldots$ & $\ldots$ & -2.4 & 6.2 & 0.140 & 0.781 & HIRES \\
\hline HAT-P-64 & 5853.92294 & 5.75 & 7.85 & 2.3 & 9.6 & 0.137 & 0.471 & HIRES \\
\hline HAT-P-64 & 5879.92287 & 20.12 & 6.98 & 17.1 & 9.6 & 0.148 & 0.960 & HIRES \\
\hline HAT-P-64 & 5882.12309 & -31.01 & 8.59 & -93.6 & 18.5 & 0.151 & 0.509 & HIRES \\
\hline HAT-P-64 & 5901.44866 & -93.15 & 44.00 & -349.0 & 88.0 & $\ldots$ & 0.331 & SOPHIE \\
\hline HAT-P-64 & 5902.42391 & 50.85 & 29.00 & -149.0 & 58.0 & $\ldots$ & 0.575 & SOPHIE \\
\hline HAT-P-64 & 5903.49529 & 104.85 & 28.00 & -16.0 & 56.0 & $\ldots$ & 0.842 & SOPHIE \\
\hline HAT-P-64 & 5904.47960 & -40.15 & 25.00 & -15.0 & 50.0 & $\ldots$ & 0.088 & SOPHIE \\
\hline HАТ-P-64 & 5904.79205 & -30.84 & 7.20 & $\ldots$ & $\ldots$ & $\ldots$ & 0.166 & HIRES \\
\hline HAT-P-64 & 5906.40764 & 35.85 & 29.00 & 172.0 & 58.0 & $\ldots$ & 0.569 & SOPHIE \\
\hline HAT-P-64 & 5908.43207 & -82.15 & 33.00 & -28.0 & 66.0 & $\ldots$ & 0.074 & SOPHIE \\
\hline HAT-P-64 & 5944.89703 & -73.94 & 7.44 & -12.3 & 9.7 & 0.145 & 0.174 & HIRES \\
\hline
\end{tabular}

Notes.

${ }^{\mathrm{a}}$ The zero-point of these velocities is arbitrary. An overall offset $\gamma_{\text {rel }}$ fitted independently to the velocities from each instrument has been subtracted.

b Internal errors excluding the component of astrophysical jitter considered in Section 3.3.

${ }^{\mathrm{c}} \mathrm{Ca}$ II HK line core emission index measured from the Keck-I/HIRES spectra following Isaacson \& Fischer (2010).

(This table is available in machine-readable form.)

well-established trend between high-equilibrium temperature and inflated radius (e.g., Fortney et al. 2007; Kovács et al. 2010; Béky et al. 2011; Enoch et al. 2011, 2012).

Including the systems presented here, a total of 67 transiting planets have now been discovered and published by HATNet. In addition to these, some 17 planets discovered by other teams have been independently detected in HATNet light curves (KELT-1, KELT-3, Kepler-6, Kepler-12, KOI-13, Qatar-1, TrES-2, TrES-3, TrES-5, WASP-2, WASP-10, WASP-13, WASP-24, WASP-33, WASP-48, XO-3, and XO-5), and more than a dozen additional transiting planets have been detected by HATNet and confirmed through follow-up observations, but have not yet been published. Altogether at least $~ 100$ transiting exoplanets have been detected by HATNet, and certainly more planets remain to be discovered among the 500 remaining candidates that have not yet been confirmed or set aside as false positives or false alarms. HATNet continues to operate in a fully autonomous manner, and will continue to produce highprecision, high-cadence time-series photometry for millions of stars over a large swath of the northern sky. Over the past 16 years it has amassed a rich database of light curves for six million stars.

The NASA TESS mission (Ricker et al. 2015) uses a set of four lenses, very similar in diameter to those used by HATNet, to survey the entire sky. Although the HATNet light curves are of lower photometric precision than TESS, the observations are made at higher spatial resolution, and are useful for identifying TESS candidates that are actually blended stellar eclipsing binary objects. The HATNet light curves may also be used in conjunction with the TESS data to search for longer-period planets than could be found in the typical 27.4 day TESS observing windows alone.

The planet HAT-P-59b presented has made for a particularly fruitful synergy between HATNet and TESS. This planet lies $10^{\circ} .4$ from the northern ecliptic pole, and is thus within the northern continuous viewing zone of TESS. It will be observed continuously for approximately one year by TESS, and we have already included seven sectors of data in our analysis of this system.

We plan to continue operating HATNet for the foreseeable future, and anticipate widening the region of parameter space to which we are sensitive to planets (i.e., toward finding subNeptune-size planets and planets with periods of several tens of days), by combining HATNet and TESS data, and by extending the time coverage of regions on the sky previously observed by HATNet.

We thank the referee, David James, for helpful comments which improved the quality of this paper. HATNet operations have been funded by NASA grants NNG04GN74G, NNX08AF23G, and NNX13AJ15G. Follow-up of HATNet targets has been partially supported through NSF grant AST- 
1108686. G.Á.B, J.H., Z.C., and K.P. acknowledge partial support from NASA grant NNX17AB61G. G.B. acknowledges support from the Hungarian Academy of Sciences, and thanks for the warm hospitality of Konkoly Observatory in carrying out some of his research. J.H. acknowledges support from NASA grant NNX14AF87G. K.P. acknowledges support from NASA grant 80NSSC18K1009. G.K. thanks the support from the National Research, Development and Innovation Office (grant K 129249). We acknowledge partial support also from the Kepler Mission under NASA Cooperative Agreement NNX13AB58A (D.W.L., PI). Data presented in this paper are based on observations obtained at the HAT station at the Submillimeter Array of SAO, and the HAT station at the Fred Lawrence Whipple Observatory of SAO. We acknowledge J. A. Johnson in supporting the Keck HIRES observations. The authors wish to acknowledge the very significant cultural role and reverence that the summit of Mauna Kea has always had within the indigenous Hawaiian community. This research has made use of Keck telescope time granted through NOAO (programs: A245Hr, A202Hr; PI: G.B) and NASA (programs: N154Hr, N133Hr, N136Hr, N143Hr, N169Hr, N186Hr; PI: G. B). Based on observations at Kitt Peak, NOAO (NOAO Prop. ID: 2015B-0156; PI: J.H.), which is operated by the Association of Universities for Research in Astronomy (AURA) under a cooperative agreement with the National Science Foundation. Based on radial velocities obtained with the SOPHIE spectrograph mounted on the $1.93 \mathrm{~m}$ telescope at Observatoire de Haute-Provence. Based on data collected at Subaru Telescope, which is operated by the National Astronomical Observatory of Japan. Based on observations made with the Nordic Optical Telescope, operated on the island of La Palma jointly by Denmark, Finland, Norway, Sweden, in the Spanish Observatorio del Roque de los Muchachos of the Intituto de Astrofísica de Canarias. Based on observations obtained with the Apache Point Observatory $3.5 \mathrm{~m}$ telescope, which is owned and operated by the Astrophysical Research Consortium. This research was made possible through the use of the AAVSO Photometric All-Sky Survey (APASS), funded by the Robert Martin Ayers Sciences Fund. This research has made use of the NASA Exoplanet Archive, which is operated by Caltech, under contract with NASA under the Exoplanet Exploration Program. This paper includes data collected with the TESS mission, obtained from the MAST data archive at the Space Telescope Science Institute (STScI). Funding for the TESS mission is provided by the NASA Explorer Program. STScI is operated by the Association of Universities for Research in Astronomy, Inc., under NASA contract NAS 5-26555.

\section{ORCID iDs}

G. Á. Bakos (iD https://orcid.org/0000-0001-7204-6727 J. D. Hartman (iD https://orcid.org/0000-0001-8732-6166 W. Bhatti (i) https://orcid.org/0000-0002-0628-0088 Z. Csubry (iD https://orcid.org/0000-0002-8423-0510

K. Penev (iD https://orcid.org/0000-0003-4464-1371

A. Bieryla (iD https://orcid.org/0000-0001-6637-5401

D. W. Latham (ib https://orcid.org/0000-0001-9911-7388

S. Quinn (1D https://orcid.org/0000-0002-8964-8377

L. A. Buchhave (1) https://orcid.org/0000-0003-1605-5666

Guillermo Torres (iD https://orcid.org/0000-0002-5286-0251

E. Falco (ib https://orcid.org/0000-0002-7061-6519

T. Szklenár (i) https://orcid.org/0000-0002-5610-7697
G. A. Esquerdo (D) https://orcid.org/0000-0002-9789-5474

A. W. Howard (ib https://orcid.org/0000-0001-8638-0320

H. Isaacson (i) https://orcid.org/0000-0002-0531-1073

G. Marcy (i) https://orcid.org/0000-0002-2909-0113

B. Sato (i) https://orcid.org/0000-0001-8033-5633

I. Boisse (iD https://orcid.org/0000-0001-8388-8399

A. Santerne (i) https://orcid.org/0000-0002-3586-1316

M. Rabus (iD https://orcid.org/0000-0003-2935-7196

D. Harbeck (10) https://orcid.org/0000-0002-8590-007X

C. McCully (i) https://orcid.org/0000-0001-5807-7893

M. E. Everett (i) https://orcid.org/0000-0002-0885-7215

E. P. Horch (10 https://orcid.org/0000-0003-2159-1463

L. Hirsch (10 https://orcid.org/0000-0001-8058-7443

S. B. Howell (iD https://orcid.org/0000-0002-2532-2853

C. X. Huang (iD https://orcid.org/0000-0003-0918-7484

\section{References}

Albrecht, S., Winn, J. N., Johnson, J. A., et al. 2012, ApJ, 757, 18 Bakos, G., Noyes, R. W., Kovács, G., et al. 2004, PASP, 116, 266 Béky, B., Bakos, G. Á., Hartman, J., et al. 2011, ApJ, 734, 109 Bieryla, A., Hartman, J. D., Bakos, G. Á., et al. 2014, AJ, 147, 84 Boisse, I., Hartman, J. D., Bakos, G. Á., et al. 2013, A\&A, 558, A86 Bouchy, F., Hébrard, G., Udry, S., et al. 2009, A\&A, 505, 853

Bovy, J., Rix, H.-W., Green, G. M., Schlafly, E. F., \& Finkbeiner, D. P. 2016, ApJ, 818, 130

Brahm, R., Jordán, A., \& Espinoza, N. 2017, PASP, 129, 034002

Brasseur, C. E., Phillip, C., Fleming, S. W., Mullally, S. E., \& White, R. L. 2019, Astrocut: Tools for Creating Cutouts of TESS Images, ascl:1905.007 Buchhave, L. A., Bakos, G. Á., Hartman, J. D., et al. 2010, ApJ, 720, 1118 Buchhave, L. A., Latham, D. W., Johansen, A., et al. 2012, Natur, 486, 375 Butler, R. P., Marcy, G. W., Williams, E., et al. 1996, PASP, 108, 500 Cardelli, J. A., Clayton, G. C., \& Mathis, J. S. 1989, ApJ, 345, 245

Charbonneau, D., Brown, T. M., Noyes, R. W., \& Gilliland, R. L. 2002, ApJ, 568,377

Claret, A. 2018, A\&A, 618, A20

Claret, A., Hauschildt, P. H., \& Witte, S. 2012, A\&A, 546, A14 Claret, A., Hauschildt, P. H., \& Witte, S. 2013, A\&A, 552, A16

Bakos, G. Á., Csubry, Z., Penev, K., et al. 2013, PASP, 125, 154

Djupvik, A. A., \& Andersen, J. 2010, in Highlights of Spanish Astrophysics V, ed. J. M. Diego et al. (Berlin: Springer), 211

Droege, T. F., Richmond, M. W., Sallman, M. P., \& Creager, R. P. 2006, PASP, 118, 1666

Enoch, B., Anderson, D. R., Barros, S. C. C., et al. 2011, AJ, 142, 86

Enoch, B., Collier Cameron, A., \& Horne, K. 2012, A\&A, 540, A99

Evans, D. W., Riello, M., De Angeli, F., et al. 2018, A\&A, 616, A4 Fûrész, G. 2008, PhD thesis, Univ. of Szeged, Hungary

Fortney, J. J., Marley, M. S., \& Barnes, J. W. 2007, ApJ, 659, 1661 Fortney, J. J., \& Nettelmann, N. 2010, SSRv, 152, 423

Gaia Collaboration, Brown, A. G. A., Vallenari, A., et al. 2018, A\&A, 616, A1 Girardi, L., Bertelli, G., Bressan, A., et al. 2002, A\&A, 391, 195

Guillot, T. 2005, AREPS, 33, 493

Hansen, B. M. S., \& Barman, T. 2007, ApJ, 671, 861

Hartman, J. D., \& Bakos, G. Á. 2016, A\&C, 17, 1

Hartman, J. D., Bakos, G. Á., Bayliss, D., et al. 2019, AJ, 157, 55

Hartman, J. D., Bakos, G. Á., Béky, B., et al. 2012, AJ, 144, 139

Hartman, J. D., Bhatti, W., Bakos, G. Á., et al. 2015, AJ, 150, 168

Hartman, J. D., Bakos, G. Á., Bhatti, W., et al. 2016, AJ, 152, 182

Horch, E. P., Bahi, L. A. P., Gaulin, J. R., et al. 2012, AJ, 143, 10

Horch, E. P., van Altena, W. F., Howell, S. B., Sherry, W. H., \& Ciardi, D. R. 2011, AJ, 141, 180

Horch, E. P., Veillette, D. R., Baena Gallé, R., et al. 2009, AJ, 137, 5057

Howell, S. B., Everett, M. E., Sherry, W., Horch, E., \& Ciardi, D. R. 2011, AJ, 142,19

Isaacson, H., \& Fischer, D. 2010, ApJ, 725, 875

Kambe, E., Sato, B., Takeda, Y., et al. 2002, PASJ, 54, 865

Kipping, D. M. 2010, MNRAS, 408, 1758

Kovács, G., Bakos, G., \& Noyes, R. W. 2005, MNRAS, 356, 557

Kovács, G., Bakos, G. Á., Hartman, J. D., et al. 2010, ApJ, 724, 866

Kovács, G., Zucker, S., \& Mazeh, T. 2002, A\&A, 391, 369

Latham, D. W., Bakos, G. Á., Torres, G., et al. 2009, ApJ, 704, 1107 
Lightkurve Collaboration, Cardoso, J. V. d. M., Hedges, C., et al. 2018, Lightkurve: Kepler and TESS Time Series Analysis in Python, ascl: 1812.013

Mallonn, M., \& Wakeford, H. R. 2017, AN, 338, 773

Mandel, K., \& Agol, E. 2002, ApJL, 580, L171

Marigo, P., Girardi, L., Bressan, A., et al. 2017, ApJ, 835, 77

Miller-Ricci, E., Seager, S., \& Sasselov, D. 2009, ApJ, 690, 1056

Noguchi, K., Aoki, W., Kawanomoto, S., et al. 2002, PASJ, 54, 855

Noyes, R. W., Hartmann, L. W., Baliunas, S. L., Duncan, D. K., \& Vaughan, A. H. 1984, ApJ, 279, 763

O’Donnell, J. E. 1994, ApJ, 422, 158

Pál, A. 2012, MNRAS, 421, 1825

Pepper, J., Pogge, R. W., DePoy, D. L., et al. 2007, PASP, 119, 923

Pollacco, D. L., Skillen, I., Collier Cameron, A., et al. 2006, PASP, 118, 1407

Queloz, D., Eggenberger, A., Mayor, M., et al. 2000, A\&A, 359, L13

Ricker, G. R., Winn, J. N., Vanderspek, R., et al. 2015, JATIS, 1, 014003

Sato, B., Hartman, J. D., Bakos, G. Á., et al. 2012, PASJ, 64, 97
Sato, B., Kambe, E., Takeda, Y., Izumiura, H., \& Ando, H. 2002, PASJ, 54,873

Scott, N. J., Howell, S. B., Horch, E. P., \& Everett, M. E. 2018, PASP, 130, 054502

Siverd, R. J., Brown, T. M., Barnes, S., et al. 2018, Proc. SPIE, 10702, $107026 \mathrm{C}$

Torres, G., Fischer, D. A., Sozzetti, A., et al. 2012, ApJ, 757, 161

Torres, G., Bakos, G. Á., Kovács, G., et al. 2007, ApJL, 666, L121

Bakos, G. Á., Torres, G., Pál, A., et al. 2010, ApJ, 710, 1724

Vogt, S. S., Allen, S. L., Bigelow, B. C., et al. 1994, Proc. SPIE, 2198, 362

Wakeford, H. R., Sing, D. K., Kataria, T., et al. 2017, Sci, 356, 628

Wang, S.-i., Hildebrand, R. H., Hobbs, L. M., et al. 2003, Proc. SPIE, 4841, 1145

Yi, S., Demarque, P., Kim, Y.-C., et al. 2001, ApJS, 136, 417

Zacharias, N., Finch, C. T., Girard, T. M., et al. 2013, AJ, 145, 44

Zechmeister, M., \& Kürster, M. 2009, A\&A, 496, 577

Zhou, G., Huang, C. X., Bakos, G. Á., et al. 2019, AJ, 158, 141 